Operation and performance of the ATLAS semiconductor tracker

This content has been downloaded from IOPscience. Please scroll down to see the full text. 2014 JINST 9 P08009

(http://iopscience.iop.org/1748-0221/9/08/P08009)

View the table of contents for this issue, or go to the journal homepage for more

Download details:

IP Address: 131.169.3.59

This content was downloaded on 02/09/2014 at 11:12

Please note that terms and conditions apply. 


\section{Operation and performance of the ATLAS semiconductor tracker}

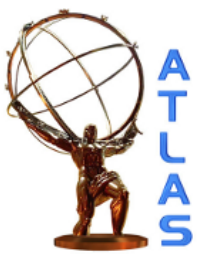

\section{The ATLAS collaboration}

E-mail: atlas.publications@cern.ch

ABSTRACT: The semiconductor tracker is a silicon microstrip detector forming part of the inner tracking system of the ATLAS experiment at the LHC. The operation and performance of the semiconductor tracker during the first years of LHC running are described. More than $99 \%$ of the detector modules were operational during this period, with an average intrinsic hit efficiency of $(99.74 \pm 0.04) \%$. The evolution of the noise occupancy is discussed, and measurements of the Lorentz angle, $\delta$-ray production and energy loss presented. The alignment of the detector is found to be stable at the few-micron level over long periods of time. Radiation damage measurements, which include the evolution of detector leakage currents, are found to be consistent with predictions and are used in the verification of radiation background simulations.

KEYWORDS: Solid state detectors; Charge transport and multiplication in solid media; Particle tracking detectors (Solid-state detectors); Detector modelling and simulations I (interaction of radiation with matter, interaction of photons with matter, interaction of hadrons with matter, etc)

ARXIV EPRINT: 1404.7473 


\section{Contents}

1 Introduction 1

2 The SCT detector 3

2.1 Layout and modules 3

2.2 Readout and data acquisition system 4

2.3 Detector services and control system 6

$\begin{array}{lll}2.4 & \text { Frequency scanning interferometry } & 7\end{array}$

2.5 Detector safety 9

3 Operation 9

3.1 Detector status 10

$\begin{array}{ll}3.2 \text { Calibration } & 12\end{array}$

$\begin{array}{lll}3.3 & \text { Timing } & 12\end{array}$

3.4 Data-taking efficiency 13

$\begin{array}{lll}3.5 & \text { Operations issues } & 14\end{array}$

4 Offline reconstruction and simulation $\quad \mathbf{1 5}$

4.1 Track reconstruction $\quad 15$

$\begin{array}{lll}4.2 & \text { Track alignment } & 17\end{array}$

$\begin{array}{lll}4.3 & \text { Simulation } & 18\end{array}$

$\begin{array}{lll}\text { 4.3.1 } & \text { Digitisation model } & 18\end{array}$

$\begin{array}{ll}\text { 4.3.2 Induced-charge model } & 19\end{array}$

$\begin{array}{ll}4.4 \text { Conditions data } & 19\end{array}$

5 Monitoring and data quality assessment 20

5.1 Online monitoring 20

5.2 Offline monitoring 21

$\begin{array}{lll}5.3 & \text { Data quality assessment } & 21\end{array}$

5.4 Prompt calibration loop 22

6 Performance 23

6.1 Detector occupancy 23

6.2 Noise 26

$\begin{array}{lll}6.3 & \text { Alignment stability } & 28\end{array}$

6.4 Intrinsic hit efficiency 31

6.5 Lorentz angle 34

6.6 Energy loss and particle identification 38

6.7 Measurement of $\delta$-ray production 39 
7 Radiation effects $\quad 41$

7.1 Simulations and predictions $\quad 43$

7.2 Detector leakage currents 44

7.2.1 Temperature and leakage-current measurement 45

7.2.2 Leakage-current evolution and comparison with predictions 45

$\begin{array}{lll}7.3 & \text { Online radiation monitor measurements } & 46\end{array}$

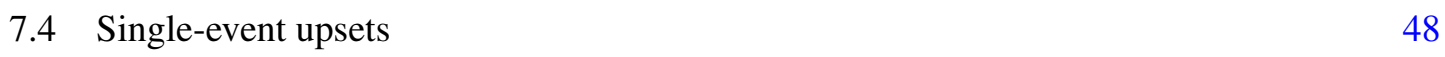

7.5 Impact of radiation on detector operation and its mitigation 49

8 Conclusions $\quad 51$

A Estimation of the bulk leakage current using models 52

$\begin{array}{ll}\text { The ATLAS collaboration } & 57\end{array}$

\section{Introduction}

The ATLAS detector [1] is a multi-purpose apparatus designed to study a wide range of physics processes at the Large Hadron Collider (LHC) [2] at CERN. In addition to measurements of Standard Model processes such as vector-boson and top-quark production, the properties of the newly discovered Higgs boson [3, 4] are being investigated and searches are being carried out for as yet undiscovered particles such as those predicted by theories including supersymmetry. All of these studies rely heavily on the excellent performance of the ATLAS inner detector tracking system. The semiconductor tracker (SCT) is a precision silicon microstrip detector which forms an integral part of this tracking system.

The ATLAS detector is divided into three main components. A high-precision toroid-field muon spectrometer surrounds electromagnetic and hadronic calorimeters, which in turn surround the inner detector. This comprises three complementary subdetectors: a silicon pixel detector covering radial distances ${ }^{1}$ between $50.5 \mathrm{~mm}$ and $150 \mathrm{~mm}$, the SCT covering radial distances from $299 \mathrm{~mm}$ to $560 \mathrm{~mm}$ and a transition radiation tracker (TRT) covering radial distances from $563 \mathrm{~mm}$ to $1066 \mathrm{~mm}$. These detectors are surrounded by a superconducting solenoid providing a $2 \mathrm{~T}$ axial magnetic field. The layout of the inner detector, showing the SCT together with the pixel detector and transition radiation tracker, is shown in figure 1 . The inner detector measures the trajectories of charged particles within the pseudorapidity range $|\eta|<2.5$. It has been designed to provide a transverse momentum resolution, in the plane perpendicular to the beam axis, of $\sigma_{p_{\mathrm{T}}} / p_{\mathrm{T}}=0.05 \% \times p_{\mathrm{T}}[\mathrm{GeV}] \oplus 1 \%$ and a transverse impact parameter resolution of $10 \mu \mathrm{m}$ for highmomentum particles in the central pseudorapidity region [1].

\footnotetext{
${ }^{1}$ ATLAS uses a right-handed coordinate system with its origin at the nominal interaction point in the centre of the detector and the $z$-axis along the beam-pipe. The $x$-axis points from the interaction point to the centre of the LHC ring and the $y$-axis points upwards. Cylindrical coordinates $(r, \phi)$ are used in the transverse plane, $\phi$ being the azimuthal angle around the beam-pipe. The pseudorapidity $\eta$ is defined in terms of the polar angle $\theta$ as $\eta=-\ln \tan (\theta / 2)$.
} 


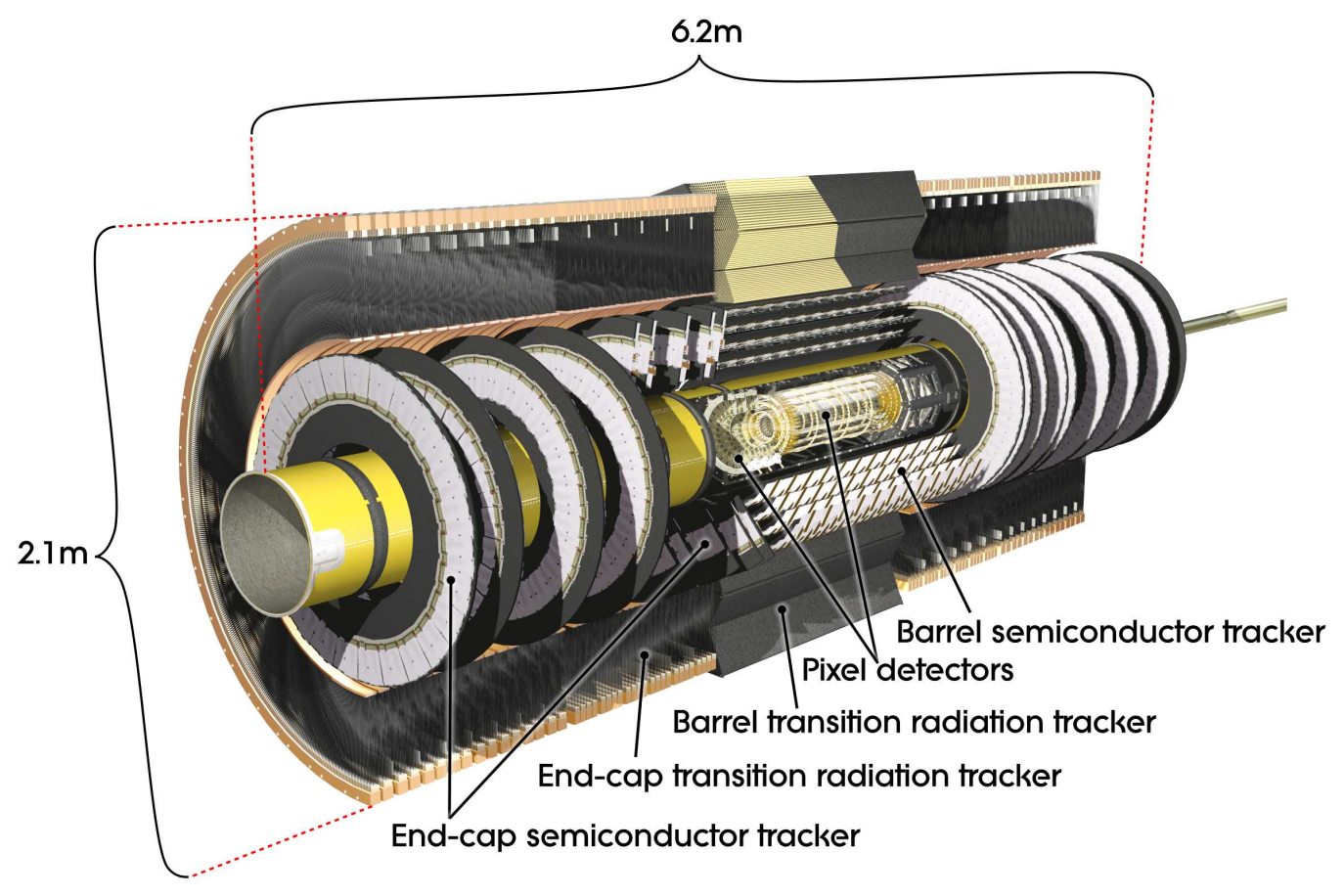

Figure 1. A cut-away view of the ATLAS inner detector.

After installation in the ATLAS cavern was completed in August 2008, the SCT underwent an extensive period of commissioning and calibration before the start of LHC proton-proton collisions in late 2009. The performance of the detector was measured using cosmic-ray data [5]; the intrinsic hit efficiency and the noise occupancy were found to be well within the design requirements. This paper describes the operation and performance of the SCT during the first years of LHC operation, from autumn 2009 to February 2013, referred to as 'Run 1'. During this period the ATLAS experiment collected proton-proton collision data at centre-of-mass energies of $\sqrt{s}=7 \mathrm{TeV}$ and $8 \mathrm{TeV}$ corresponding to integrated luminosities [6] of $5.1 \mathrm{fb}^{-1}$ and $21.3 \mathrm{fb}^{-1}$ respectively, together with small amounts at $\sqrt{s}=900 \mathrm{GeV}$ and $2.76 \mathrm{TeV}$. In addition, $158 \mu \mathrm{b}^{-1}$ of lead-lead collision data at a nucleon-nucleon centre-of-mass energy of $2.76 \mathrm{TeV}$ and $30 \mathrm{nb}^{-1}$ of proton-lead data at a nucleon-nucleon centre-of-mass energy of $5 \mathrm{TeV}$ were recorded. The collision data are used to measure the intrinsic hit efficiency of the silicon modules and the Lorentz angle. Compared with the previous results using cosmic-ray data [5], the efficiency measurements are now extended to the endcap regions of the detector, and the large number of tracks has allowed the Lorentz angle to be studied in more detail. In addition, studies of energy loss and $\delta$-ray production in the silicon have been performed.

The layout of this paper is as follows. The main components of the SCT are described briefly in section 2. The operation of the detector is discussed in section 3. Offline reconstruction and simulation are outlined in section 4, and monitoring and data quality assessment discussed in section 5 . Section 6 presents performance results, including detector occupancy in physics running, noise occupancy, alignment, efficiency, measurements of the Lorentz angle and energy loss in the silicon and a study of $\delta$-ray production in silicon. Finally, section 7 describes the effects of radiation on the detector. 


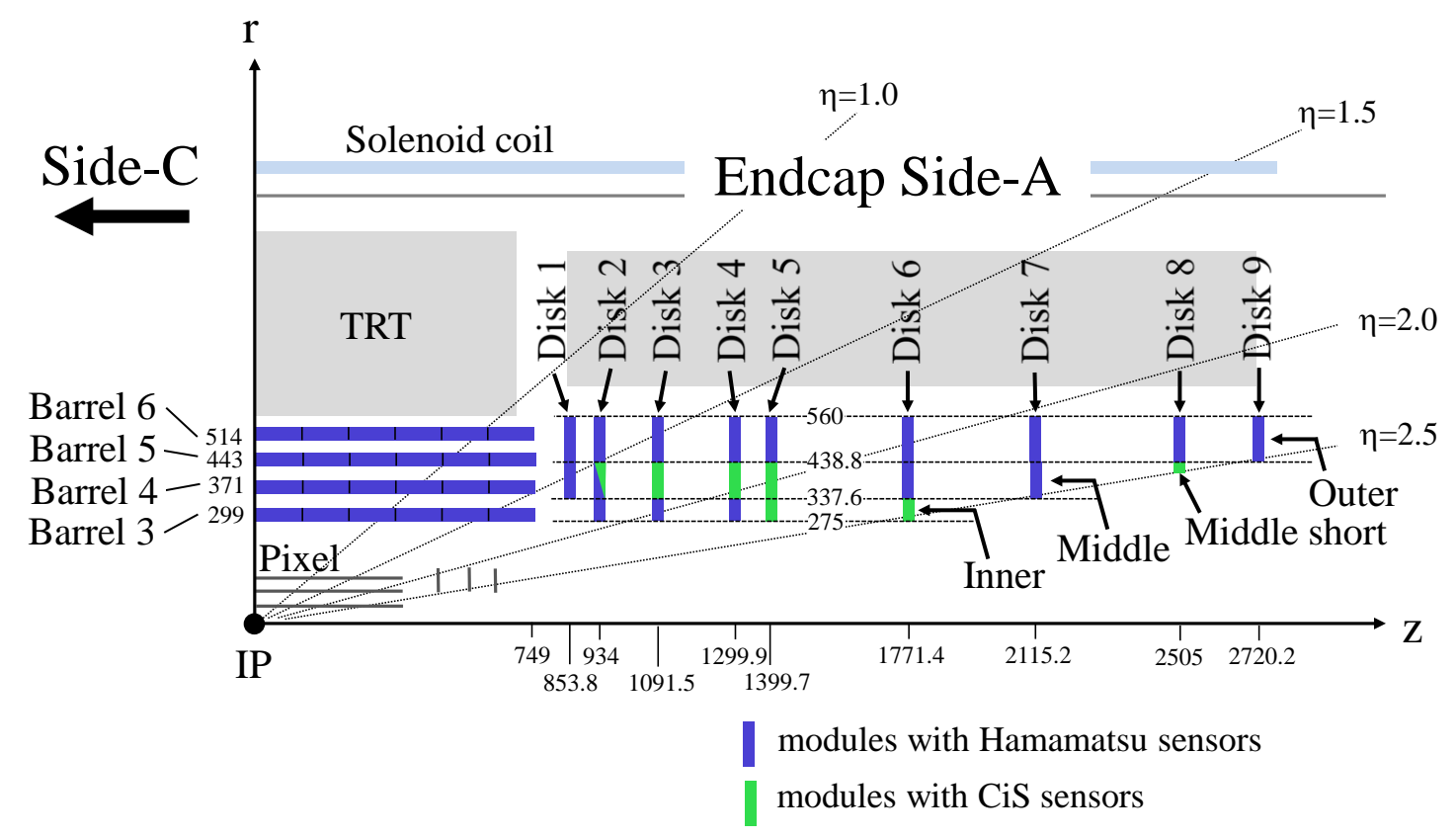

Figure 2. A schematic view of one quadrant of the SCT. The numbering scheme for barrel layers and endcap disks is indicated, together with the radial and longitudinal coordinates in millimetres. The disks comprise one, two or three rings of modules, referred to as inner, middle and outer relative to the beam pipe. The figure distinguishes modules made from Hamamatsu (blue) or CiS (green) sensors; the middle ring of disk 2 contains modules of both types. The two sets of endcap disks are distinguished by the labels A (positive $z$ ) and $\mathrm{C}$ (negative $z$ ).

\section{The SCT detector}

The main features of the SCT are described briefly in this section; full details can be found in ref. [1].

\subsection{Layout and modules}

The SCT consists of 4088 modules of silicon-strip detectors arranged in four concentric barrels (2112 modules) and two endcaps of nine disks each (988 modules per endcap), as shown in figure 2. Each barrel or disk provides two strip measurements at a stereo angle which are combined to build space-points. The SCT typically provides eight strip measurements (four space-points) for particles originating in the beam-interaction region. The barrel modules [7] are of a uniform design, with strips approximately parallel to the magnetic field and beam axis. Each module consists of four rectangular silicon-strip sensors [8] with strips with a constant pitch of $80 \mu \mathrm{m}$; two sensors on each side are daisy-chained together to give 768 strips of approximately $12 \mathrm{~cm}$ in length. A second pair of identical sensors is glued back-to-back with the first pair at a stereo angle of $40 \mathrm{mrad}$. The modules are mounted on cylindrical supports such that the module planes are at an angle to the tangent to the cylinder of $11^{\circ}$ for the inner two barrels and $11.25^{\circ}$ for the outer two barrels, and overlap by a few millimetres to provide a hermetic tiling in azimuth. 
Each endcap disk consists of up to three rings of modules [9] with trapezoidal sensors. The strip direction is radial with constant azimuth and a mean pitch of $80 \mu \mathrm{m}$. As in the barrel, sensors are glued back-to-back at a stereo angle of $40 \mathrm{mrad}$ to provide space-points. Modules in the outer and middle rings consist of two daisy-chained sensors on each side, whereas those in the inner rings have one sensor per side.

All sensors are $285 \mu \mathrm{m}$ thick and are constructed of high-resistivity n-type bulk silicon with p-type implants. Aluminium readout strips are capacitatively coupled to the implant strips. The barrel sensors and $75 \%$ of the endcap sensors were supplied by Hamamatsu Photonics, ${ }^{2}$ while the remaining endcap sensors were supplied by CiS. ${ }^{3}$ Sensors supplied by the two manufacturers meet the same performance specifications, but differ in design and processing details [8]. The majority of the modules are constructed from silicon wafers with crystal lattice orientation (Miller indices) $<111>$. However, a small number of modules in the barrel $(\sim 90)$ use wafers with $<100>$ lattice orientation. For most purposes, sensors from the different manufacturers or with different crystal orientation are indistinguishable, but differences in e.g. noise performance have been observed, as discussed in section 6.2.

Measurements often require a selection on the angle of a track incident on a silicon module. The angle between a track and the normal to the sensor in the plane defined by the normal to the sensor and the local $x$-axis (i.e. the axis in the plane of the sensor perpendicular to the strip direction) is termed $\phi_{\text {local }}$. The angle between a track and the normal to the sensor in the plane defined by the normal to the sensor and the local $y$-axis (i.e. the axis in the plane of the sensor parallel to the strip direction) is termed $\theta_{\text {local }}$.

\subsection{Readout and data acquisition system}

The SCT readout system was designed to operate with $0.2 \%-0.5 \%$ occupancy in the 6.3 million sampled strips, for the original expectations for the LHC luminosity of $1 \times 10^{34} \mathrm{~cm}^{-2} \mathrm{~s}^{-1}$ and pileup $^{4}$ of up to 23 interactions per bunch crossing. The strips are read out by radiation-hard front-end ABCD chips [10] mounted on copper-polyimide flexible circuits termed the readout hybrids. Each of the 128 channels of the $\mathrm{ABCD}$ has a preamplifier and shaper stage; the output has a shaping time of $\sim 20 \mathrm{~ns}$ and is then discriminated to provide a binary output. A common discriminator threshold is applied to all 128 channels, normally corresponding to a charge of $1 \mathrm{fC}$, and settable by an 8-bit DAC. To compensate for variations in individual channel thresholds, each channel has its own 4-bit DAC (TrimDAC) used to offset the comparator threshold and enable uniformity of response across the chip. The step size for each TrimDAC setting can be set to four different values, as the spread in uncorrected channel-to-channel variations is anticipated to increase with total ionising dose.

The binary output signal for each channel is latched to the $40 \mathrm{MHz}$ LHC clock and stored in a 132-cell pipeline; the pipeline records the hit sequence for that channel for each clock cycle over $\sim 3.2 \mu$ s. Following a level-1 trigger, data for the preceding, in-time and following bunch crossings are compressed and forwarded to the off-detector electronics. Several modes of data compression have been used, as specified by the hit pattern for these three time bins:

\footnotetext{
${ }^{2}$ Hamamatsu Photonics Co. Ltd.,1126-1 Ichino-cho, Hamamastu, Shizuoka 431-3196, Japan.

${ }^{3}$ CiS Institut für Mikrosensorik gGmbH, Konrad-Zuse-Straße 14, 99099 Erfurt, Germany.

${ }^{4}$ The term pile-up refers to multiple $p p$ interactions per bunch crossing.
} 
- Any hit mode; channels with a signal above threshold in any of the three bunch crossings are read out. This mode is used when hits outside of the central bunch crossing need to be recorded, for example to time in the detector or to record cosmic-ray data.

- Level mode (X1X); only channels with a signal above threshold in the in-time bunch crossing are read out. This is the default mode used to record data in 2011-2013, when the LHC bunch spacing was $50 \mathrm{~ns}$, and was used for all data presented in this paper unless otherwise stated.

- Edge mode (01X); only channels with a signal above threshold in the in-time bunch crossing and no hit in the preceding bunch crossing are read out. This mode is designed for $25 \mathrm{~ns}$ LHC bunch spacing, to remove hits from interactions occurring in the preceding bunch crossing.

The off-detector readout system [11] comprises $909 \mathrm{U}$ readout-driver boards (RODs) and 90 back-of-crate (BOC) cards, housed in eight $9 \mathrm{U}$ VME crates. A schematic diagram of the data acquisition system is shown in figure 3. Each ROD processes data for up to 48 modules, and the BOC provides the optical interface between the ROD and the modules; the BOC also transmits the reformatted data for those 48 modules to the ATLAS data acquisition (DAQ) chain via a single fibre known as an 'S-link'. There is one timing, trigger and control (TTC) stream per module from the BOC to the module, and two data streams returned from each module corresponding to the two sides of the module. The transmission is based on vertical-cavity surface-emitting lasers (VCSELs) operating at a wavelength of $850 \mathrm{~nm}$ and uses radiation-hard fibres [12]. The TTC data are broadcast at $40 \mathrm{Mb} / \mathrm{s}$ to the modules via 12-way VCSEL arrays, and converted back to electrical signals by silicon $p-i-n$ diodes on the on-detector optical harnesses. Data are optically transmitted back from the modules at $40 \mathrm{Mb} / \mathrm{s}$ and received by arrays of silicon $p-i-n$ diodes on the BOC.

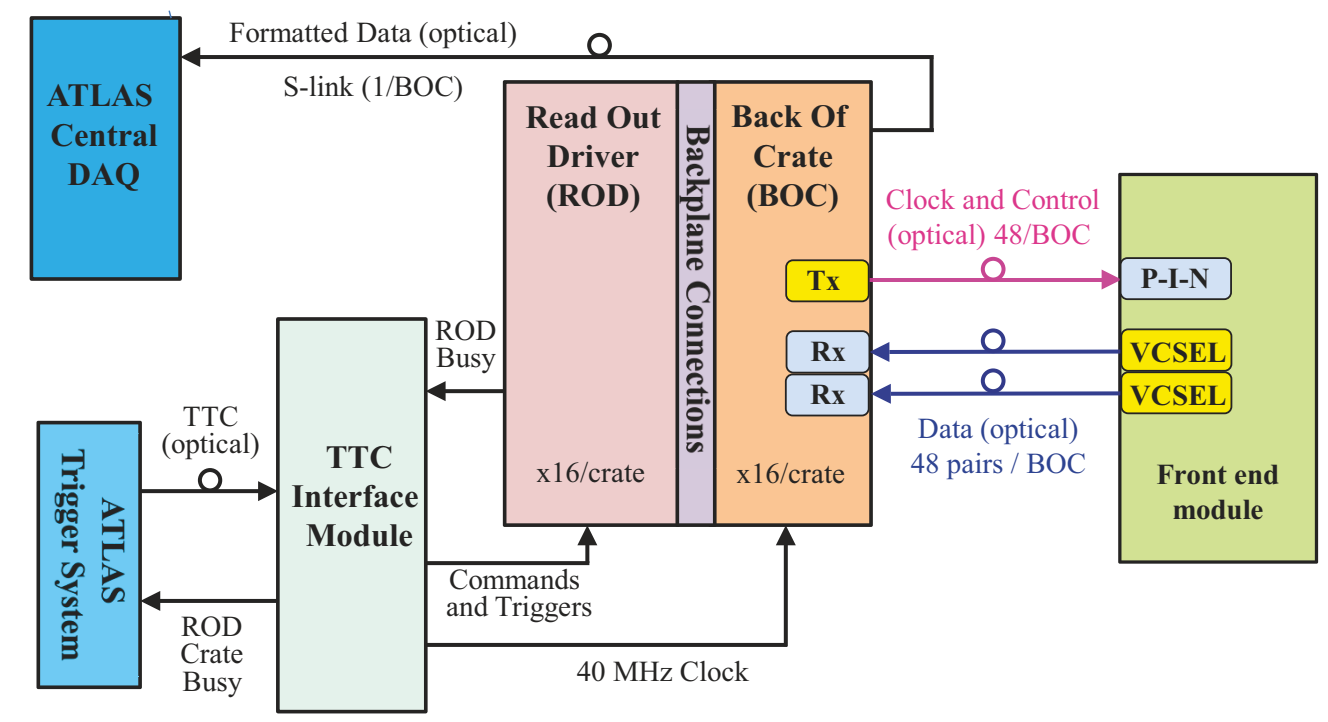

Figure 3. A schematic diagram of the SCT data acquisition hardware showing the main connections between components. 
Redundancy is implemented for both the transmitting (TX) and receiving (RX) optical links, in case of fibre breaks, VCSEL failures or diode problems. Redundancy is built into the TX system by having electrical links from one module to its neighbour. If a module loses its TTC signal for any reason, an electrical control line can be set which results in the neighbouring module sending a copy of its TTC data to the module with the failed signal, without any impact on operation. For the data links, one side of the module can be configured to be read out through the other link. Although readout of both sides of the module though one RX link inevitably reduces the readout bandwidth, this is still within design limits for SCT operation. For the barrel modules, readout of both sides through one link also results in the loss of one ABCD chip on the re-routed link because the full redundancy scheme was not implemented due to lack of space on the readout hybrid.

Table 1 shows the number of optical links configured to use the redundancy mechanism at the end of data-taking in February 2013. The RX links configured to read out both module sides were mainly due to connectivity defects during installation of the SCT, and the number remained stable throughout Run 1 . The use of the TX redundancy mechanism varied significantly due to VCSEL failures, discussed in section 3.5.

Table 1. Numbers of optical links configured to use the redundancy mechanism in February 2013, in the barrel, the endcaps and the whole SCT. The last column shows the corresponding fraction of all links in the detector.

\begin{tabular}{|lcccc|}
\hline \multicolumn{5}{|c|}{ Links using redundancy mechanism } \\
\hline Links & Barrel & Endcaps & SCT & Fraction [\%] \\
\hline TX & 9 & 5 & 14 & 0.3 \\
RX & 41 & 91 & 132 & 1.6 \\
\hline
\end{tabular}

\subsection{Detector services and control system}

The SCT, together with the pixel detector, is cooled by a bi-phase evaporative system [13] which is designed to deliver $\mathrm{C}_{3} \mathrm{~F}_{8}$ fluid at $-25^{\circ} \mathrm{C}$ via 204 independent cooling loops within the lowmass cooling structures on the detector. The target temperature for the SCT silicon sensors after irradiation is $-7^{\circ} \mathrm{C}$, which was chosen to moderate the effects of radiation damage. An interlock system, fully implemented in hardware, using two temperature sensors located at the end of a half cooling-loop for 24 barrel modules or either 10 or 13 endcap modules, prevents the silicon modules from overheating in the event of a cooling failure by switching off power to the associated channels within approximately one second. The cooling system is interfaced to and monitored by the detector control system, and is operated independently of the status of SCT operation.

The SCT detector control system (DCS) [14] operates within the framework of the overall ATLAS DCS [15]. Custom embedded local monitor boards [16], designed to operate in the strong magnetic field and high-radiation environment within ATLAS, provide the interface between the detector hardware and the readout system. Data communication through controller area network ${ }^{5}$ (CAN) buses, alarm handling and data display are handled by a series of PCs running the commercial controls software PVSS-II. ${ }^{6}$

${ }^{5}$ CAN in Automation, Kontumazgarten 3, DE-90429, Nürenburg, Germany.

${ }^{6}$ ETM professional control GmbH, Marketstrasse 3, A-7000 Eisenstadt, Austria. 
The DCS is responsible for operating the power-supply system: setting the high-voltage (HV) supplies to the voltage necessary to deplete the sensors and the low-voltage (LV) supplies for the read-out electronics and optical-link operation. It monitors voltages and currents, and also environmental parameters such as temperatures of sensors, support structures and cooling pipes, and the relative humidity within the detector volume. The DCS must ensure safe and reliable operation of the SCT, by taking appropriate action in the event of failure or error conditions.

The power-supply system is composed of 88 crates, each controlled by a local monitor board and providing power for $48 \mathrm{LV} / \mathrm{HV}$ channels. For each channel, several parameters are monitored and controlled, amounting to around 2500 variables per crate. A total of 16 CAN buses are needed to ensure communication between the eight DCS PCs and the power-supply crates. The environmental monitoring system reads temperatures and humidities of about 1000 sensors scattered across the SCT volume, and computes dew points. Temperature sensors located on the outlet of the cooling loops are read in parallel by the interlock system, which can send emergency stop signals to the appropriate power-supply crate by means of an interlock-matrix programmable chip in the unlikely event of an unplanned cooling stoppage. All environmental sensors are read by local monitor boards connected to two PCs, each using one CAN bus.

The SCT DCS is integrated into the ATLAS DCS via a finite state machine structure (where the powering status of the SCT can be one of a finite number of states) forming a hierarchical tree. States of the hardware are propagated up the tree and combined to form a global detector state, while commands are propagated down from the user interfaces. Alarm conditions can be raised when the data values from various parameters of the DCS go outside of defined limits.

Mitigation of electromagnetic interference and noise pickup from power lines is critical for the electrical performance of the detector. Details of the grounding and shielding of the SCT are described in ref. [17].

\subsection{Frequency scanning interferometry}

Frequency scanning interferometry (FSI) is a novel technique developed to monitor the alignment stability of the detector by measuring distances between fiducial points on the support structure with high precision [18]. It provides information to augment track-based alignment by determining the internal distortions of the SCT structure on short, medium and long timescales. Lengths are measured using 842 laser interferometers arranged in a geodetic grid covering the detector; the grid layout is shown in figure $4 \mathrm{a}$. The lengths of the grid lines are measured in real time and compared to a reference length provided by an off-detector reference system in a controlled environment. Two lasers are used to scan the frequencies in opposite directions (increasing, decreasing) to cancel drift errors. The light from each laser is split into two beams to be sent simultaneously to the endcap and barrel sections of the system. The beams are then split close to the detector into the hundreds of interferometers. The same light is also sent to the reference system for later analysis. The working principle of the individual interferometers is shown in figure $4 \mathrm{~b}$. The distance is measured between two components of each interferometer: a 'quill' which contains the light delivery and return fibres, and a distant retro-reflector. The wide-angle beam emerging from the quill provides tolerance to small misalignments which may occur during the planned ten-year operational lifetime. As a trade off, the interferometer provides a return signal of around only $1 \mathrm{pW}$ per $\mathrm{mW}$ of input power, for a $1 \mathrm{~m}$ interferometer length. 


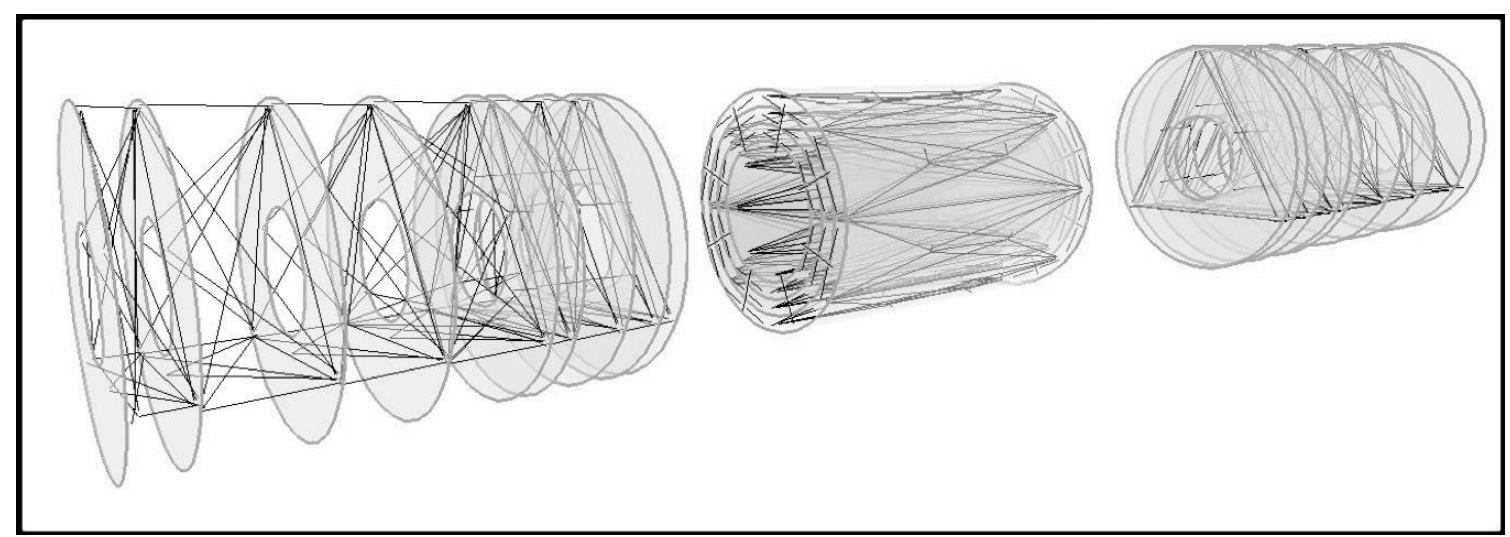

(a)

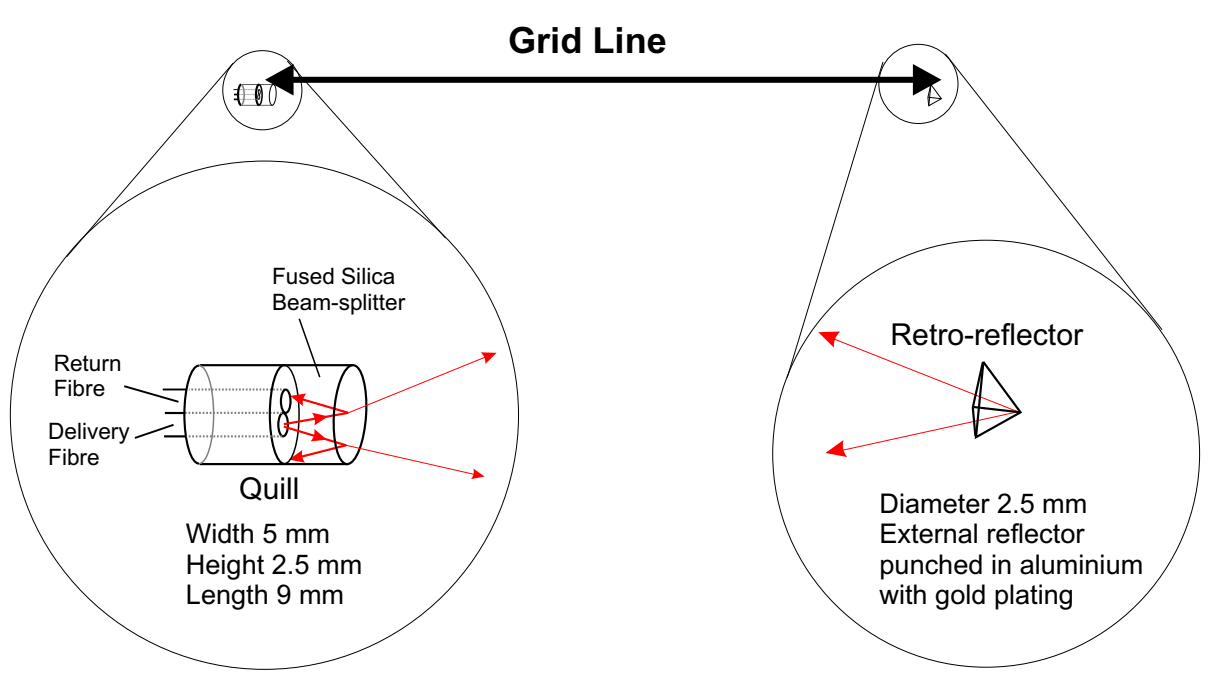

(b)

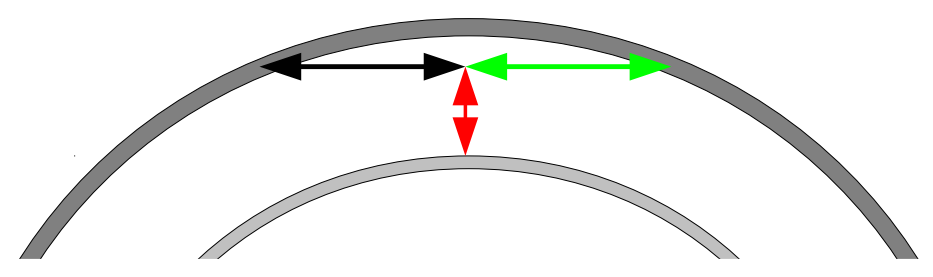

(c)

Figure 4. (a) The FSI grid layout across the SCT volume. (b) The grid-line interferometer design for the FSI system. (c) The arrangement of each group of three grid-line interferometers on the barrel flange. The colours used here for each line in the assembly are replicated in section 6.3. 
The FSI can be operated to measure either the absolute or relative phase changes of the interference patterns. In the absolute mode, absolute distances are measured with micron-level precision over long periods. In the relative mode, the relative phase change of each interferometer is monitored over short periods with a precision on distance approaching $50 \mathrm{~nm}$. Both modes can be used with all 842 grid lines.

The FSI has been in operation since 2009. The nominal power to the interferometers is $1 \mathrm{~mW}$ per interferometer; however, during early operation, with low trip levels on the leakage current from the sensors, this power was inducing too much leakage current in the silicon modules. For this reason, the power output of the two main lasers was reduced to allow for safe SCT operation. As the leakage current increased because of radiation damage, this limitation was relaxed and the trip levels increased. In the current setup, the optical power delivered only allows analysis of data from the 144 interferometers that measure distances between the four circular flanges at each end of the barrel. These interferometers are grouped into 48 assemblies of three interferometers each, which monitor displacements between the carbon-fibre support cylinders in adjacent barrel layers, as illustrated in figure $4 \mathrm{c}$.

\subsection{Detector safety}

The ATLAS detector safety system [19] protects the SCT and all other ATLAS detector systems by bringing the detector to a safe state in the event of alarms arising from cooling system malfunction, fire, smoke or the leakage of gases or liquids. Damage to the silicon sensors could also arise as a result of substantial charge deposition during abnormal beam conditions. Beam conditions are monitored by two devices based on radiation-hard polycrystalline chemical vapour deposition diamond sensors.

The beam conditions monitor (BCM) $[1,20,21]$ consists of two stations, forward and backward, each with four modules located at $z= \pm 1.84 \mathrm{~m}$ and a radius of $5.5 \mathrm{~cm}$. Each module has two diamond sensors of $1 \times 1 \mathrm{~cm}^{2}$ surface area and $500 \mu \mathrm{m}$ thickness mounted back-to-back. The $1 \mathrm{~ns}$ signal rise time allows discrimination of particle hits due to collisions (in-time) from background (out-of-time). Background rates for the two circulating beams can be measured separately, and are used to assess the conditions before ramping up the high voltage on the SCT modules.

The beam loss monitor (BLM) [22] consists of 12 diamond sensors located at a radius of $6.5 \mathrm{~cm}$ at $z \simeq \pm 3.5 \mathrm{~m}$. The radiation-induced currents in the sensors are averaged over various time periods ranging from $40 \mu \mathrm{s}$ to $84 \mathrm{~s}$. The BLM triggers a fast extraction of the LHC beams if a high loss rate is detected, i.e. if the current averaged over the shortest integration time of $40 \mu$ s exceeds a preset threshold simultaneously in two modules on each side of the interaction point.

\section{Operation}

The LHC delivered proton-proton collision data at $\sqrt{s}=7 \mathrm{TeV}$ corresponding to integrated luminosities of $47 \mathrm{pb}^{-1}$ and $5.6 \mathrm{fb}^{-1}$ in 2010 and 2011 respectively, and a further $23.3 \mathrm{fb}^{-1}$ at $\sqrt{s}=$ $8 \mathrm{TeV}$ in 2012 . There were also three periods of running with heavy ions instead of protons, each approximately one month long. The SCT has been operational throughout all data-taking periods. It delivered high-quality tracking data for $99.9 \%, 99.6 \%$ and $99.1 \%$ of the delivered proton-proton luminosity in 2010, 2011 and 2012 respectively. 
The typical cycle of daily LHC operations involves a period of beam injection and energy ramp, optimisation for collisions, declaration of collisions with stable conditions, a long period of physics data-taking, and finally a dump of the beam. The SCT remains continuously powered regardless of the LHC status. In the absence of stable beam conditions at the LHC, the SCT modules are biased at a reduced high voltage of $50 \mathrm{~V}$ to ensure that the silicon sensors are only partially depleted; in the unlikely event of a significant beam loss, this ensures that a maximum of $50 \mathrm{~V}$ is applied temporarily across the strip oxides, which is not enough to cause electrical breakdown. Normal data-taking requires a bias voltage of $150 \mathrm{~V}$ on the silicon in order to maximise hit efficiencies for tracking, and the process of switching the SCT from standby at $50 \mathrm{~V}$ to on at $150 \mathrm{~V}$ is referred to as the 'warm start'. Once the LHC declares stable beam conditions, the SCT is automatically switched on if the LHC collimators are at their nominal positions for physics, if the background rates measured in BCM, BLM and the ATLAS forward detectors are low enough, and if the SCT hit occupancy with $50 \mathrm{~V}$ is consistent with the expected luminosity.

\subsection{Detector status}

The evaporative cooling system provided effective cooling for the SCT as well as the pixel subdetector throughout the Run 1 period. The system was usually operated continuously apart from 10-20 days of maintenance annually while the LHC was shut down. Routine maintenance (e.g. compressor replacements) could be performed throughout the year without affecting the operation, as only four of the available seven compressors were actually required for operation. In the first year, the system had several problems with compressors, leaks of fluid and malfunctioning valves. However, operation in 2011 and 2012 was significantly more stable, following increased experience and optimisation of maintenance procedures. For example, in 2011 there were only two problems coming from the system itself out of 19 cooling stops. The number of active cooling loops as a function of time during Run 1 is shown in figure 5a. Figure 5b shows the mean temperatures for each barrel and each endcap ring in the same time period, as measured by sensors mounted on the hybrid of each module. The inner three barrels were maintained at a hybrid temperature of approximately $2^{\circ} \mathrm{C}$, while the outermost barrel and the endcap disk temperatures were around $7^{\circ} \mathrm{C}$. The mean temperatures of each layer were stable within about one degree throughout the three-year period.

The detector operative fraction was consistently high, with at least $99 \%$ of the SCT modules functional and available for tracking throughout 2010 to 2013. Table 2 shows the numbers of disabled detector elements at the end of data-taking in February 2013. The numbers are typical and changed minimally during the Run 1 period. The number of disabled strips (mainly due to high noise or unbonded channels) and non-functioning chips is negligible and the largest contribution is due to disabled modules, as detailed in table 3. Half of the disabled modules are due to one cooling loop permanently disabled as a result of an inaccessible leak in that loop. Fortunately this only affects one quadrant of one of the outermost endcap disks, and has negligible impact on tracking performance. The remaining disabled modules are predominantly due to on-detector connection issues. 

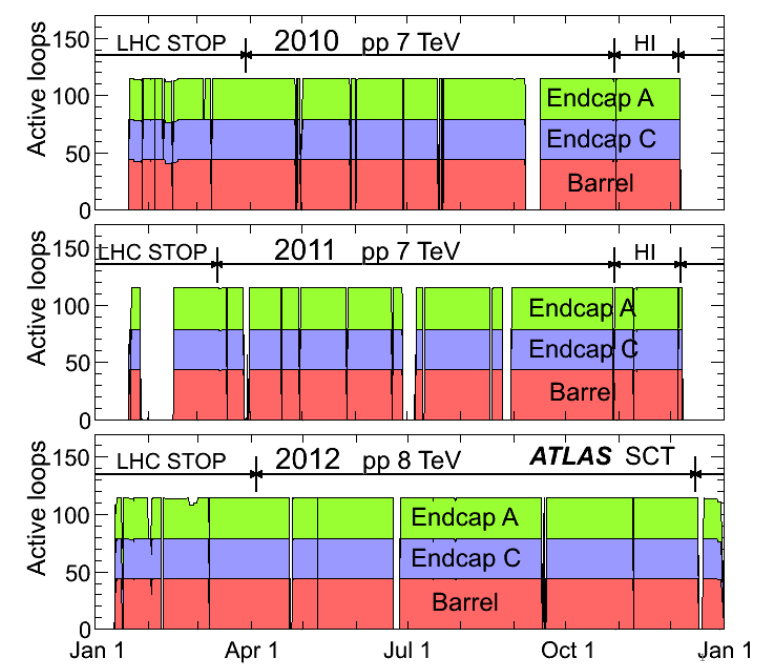

(a)

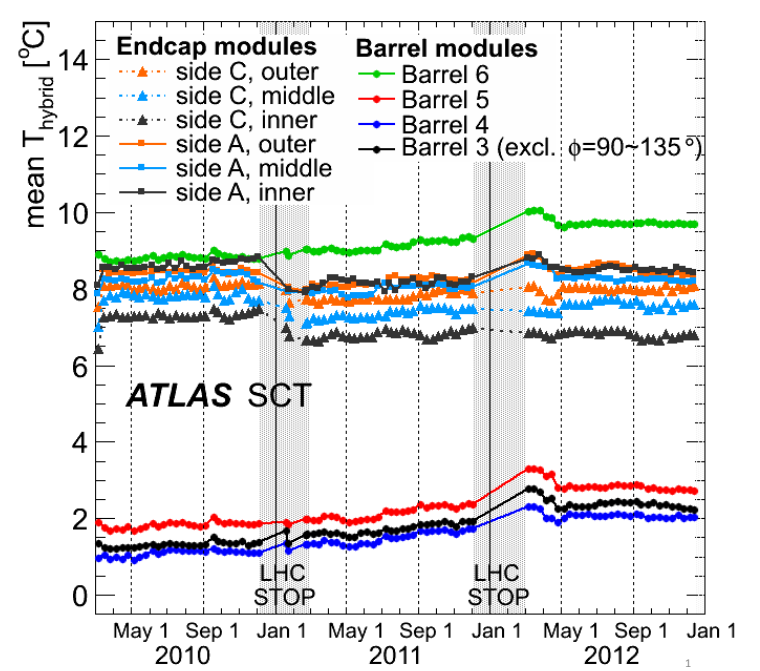

(b)

Figure 5. (a) Number of active cooling loops during 2010 to 2012. Periods of $p p$ collisions, heavy-ion (HI) running and LHC shutdown periods are indicated. The periods of a few days with no active cooling loops correspond to short technical stops of the LHC. (b) Mean hybrid temperature, $T_{\text {hybrid, as a function of }}$ time (averaged over intervals of ten days). Values are shown for each barrel, and for each ring of endcap modules, separately for endcap sides $\mathrm{A}$ and $\mathrm{C}$. The values for barrel 3 exclude modules served by one cooling loop $\left(\phi \sim 90^{\circ}-135^{\circ}\right)$, which has a temperature consistently about $4^{\circ} \mathrm{C}$ higher. The grey bands indicate the extended periods without LHC beam around the end of each year.

Table 2. Numbers of disabled SCT detector elements in February 2013, in the barrel, the endcaps and the whole SCT. The last column shows the corresponding fraction of all elements in the detector. The numbers of chips (strips) exclude those in disabled modules (modules and chips).

\begin{tabular}{|lcccc|}
\hline \multicolumn{5}{c|}{ Number of disabled elements } \\
\hline Component & Barrel & Endcaps & SCT & Fraction [\%] \\
\hline Modules & 11 & 19 & 30 & 0.73 \\
Chips & 38 & 17 & 55 & 0.11 \\
Strips & 4111 & 7252 & 11363 & 0.18 \\
\hline
\end{tabular}

Table 3. Numbers of disabled modules in February 2013 classified according to reason. The first three columns show the numbers of modules affected by each issue for the barrel, endcaps and the whole SCT, while the final column shows the corresponding fraction of all modules in the detector.

\begin{tabular}{|lcccc|}
\hline \multicolumn{5}{c|}{ Number of disabled modules } \\
\hline & Barrel & Endcaps & SCT & Fraction [\%] \\
\hline Cooling & 0 & 13 & 13 & 0.32 \\
LV & 6 & 1 & 7 & 0.17 \\
HV & 1 & 5 & 6 & 0.15 \\
Readout & 4 & 0 & 4 & 0.10 \\
\hline Total & 11 & 19 & 30 & 0.73 \\
\hline
\end{tabular}




\subsection{Calibration}

Calibrations were regularly performed between LHC fills. The principle aim is to impose a $1 \mathrm{fC}$ threshold across all chips to ensure low noise occupancy $\left(<5 \times 10^{-4}\right)$ and yet high hit efficiency ( $>99 \%$ ) for each channel. Calibrations also provide feedback to the offline event reconstruction, and measurements of electrical parameters such as noise for use in studies of detector performance. There are three categories of calibration tests:

- Electrical tests to optimise the chip configuration and to measure noise and gain, performed every few days.

- Optical tests to optimise parameters relevant to the optical transmission and reception of data between the back-of-crate cards and the modules, performed daily when possible.

- Digital tests to exercise and verify the digital functionality of the front-end chips, performed occasionally.

The principle method of electrical calibration is a threshold scan. A burst of triggers is issued and the occupancy (fraction of triggers which generate a hit) is measured while a chip parameter, usually the discriminator setting, is varied in steps. Most electrical calibrations involve injecting a known amount of charge into the front-end of the chip by applying a voltage step across a calibration capacitor. The response to the calibration charge when scanning the discriminator threshold is parameterised using a complementary error function. The threshold at which the occupancy is $50 \%$ (vt50) corresponds to the median of the injected charge, while the Gaussian spread gives the noise after amplification. The process is repeated for several calibration charges (typically $0.5 \mathrm{fC}$ to $8 \mathrm{fC}$ ). The channel gain is extracted from the dependence of vt50 on input charge (slope of the response curve) and the input equivalent noise charge (ENC) is obtained by dividing the output noise by the gain.

\subsection{Timing}

The trigger delivered to each SCT module must be delayed by an amount equal to the pipeline length minus all external delays (e.g. those incurred by the trigger system and cable delays) in order to select the correct position in the pipeline. Prior to collision data-taking, the trigger delay to each of the 4088 modules was adjusted to compensate for the different cable and fibre lengths between the optical transmitter on the BOC and the point of trigger signal distribution on the module, and for the different times-of-flight for particles from collisions, depending on the geometric location of the module. Further adjustments were applied using collision data.

On receipt of a level-1 trigger, the contents of three consecutive pipeline bins are sampled, and the timing is considered optimal if the hit pattern arising from a particle from a collision in ATLAS gives 01X (nothing in first bin, a hit in the middle bin, and either in the third bin). The procedure for timing in the SCT is to take physics data with $p p$ collisions while stepping through the timing delay in (typically) $5 \mathrm{~ns}$ steps. The optimal delay is derived from the mid-point of the time-delay range for which the fraction of recorded hits on reconstructed tracks satisfying $01 \mathrm{X}$ is maximal. In general, such a timing scan was performed and any necessary timing adjustments applied during the first collision runs in each year. 


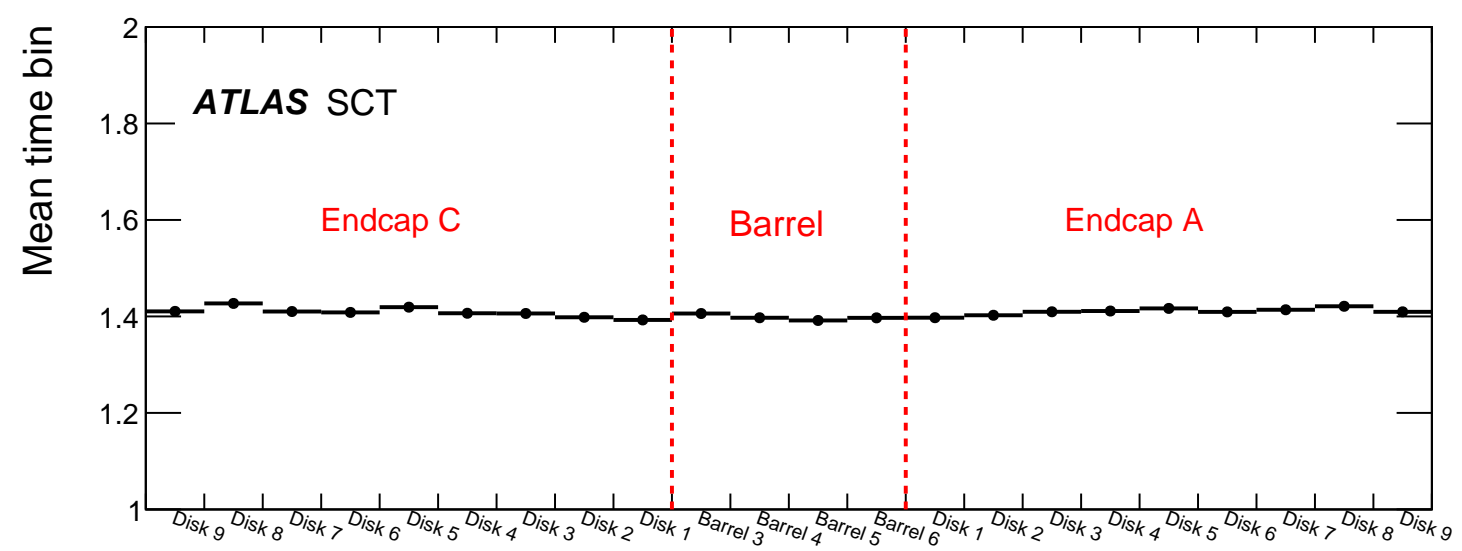

Figure 6. The mean of the three-bin timing distribution across all SCT layers; 010 and 011 hits correspond to 1.0 and 1.5 in the plot, respectively. The error bars represent the r.m.s. spread of mean time bin for modules in that layer.

A verification of the timing is performed by a check of the hit pattern in the three sampled time bins during data-taking. For each module, a three-bin histogram is filled according to whether a hit-on-track is above threshold in each time bin. After timing in, only the time-bin patterns 010 and 011 are significantly populated. The mean value of the histogram would be 1.0 if all hits were 010 and 1.5 if all hits were 011 , since the second and third bins would be equally populated. The mean value per layer for a high-luminosity $p p$ run in October 2012 is shown in figure 6; the error bars represent the r.m.s. spread of mean time bin for modules in that layer. The plot is typical, and illustrates that the timing of the SCT is uniform. Around $71 \%$ of the hits-on-track in this run have a time-bin pattern of 011 . This fraction varies by about $1 \%$ from layer to layer and $2.5 \%$ for modules within a layer.

\subsection{Data-taking efficiency}

There are three potential sources of data-taking inefficiency: the time taken to switch on the SCT upon the declaration of stable beam conditions, errors from the chips which flag that data fragments from those chips cannot be used for tracking, and a signal (known as 'busy') from the SCT DAQ which inhibits ATLAS data-taking due to a DAQ fault. Of these, the busy signal was the dominant cause of the $\sim 0.9 \%$ loss in data-taking efficiency in 2012 ; the warm start typically took 60 seconds, which was shorter than the time taken by some other subsystems in ATLAS; the chip errors effectively reduced the detector acceptance but had little impact on overall data-taking efficiency.

The presence of ROD busy signals was mainly due to the very high occupancy and trigger rates experienced during 2012; the mean number of interactions per bunch crossing during $p p$ collisions routinely exceeded the original design expectations of around 23, often reaching 30 or more with level-1 trigger rates of typically $60-80 \mathrm{kHz}$. Although the bandwidth of the DAQ was sufficient to cope with these conditions, the high occupancy and rate exposed shortcomings in the on-ROD processing and decoding of the data which led to an increased rate of disabled data links and also ROD busy signals. Specifically, problems within the ROD firmware were exposed by optical noise 
on the data-link inputs (often associated with failures of the TX optical transmitters and also high current from some endcap modules with CiS sensors), and by specific high-occupancy conditions. It is anticipated that these issues will be resolved in later ATLAS runs by upgrades to the ROD firmware. The impact on data-taking efficiency was mitigated by introducing the ability to remove a ROD which holds busy indefinitely from the ATLAS DAQ, reconfigure the affected modules, and then to re-integrate the ROD without interruption to ATLAS data-taking.

The DAQ may flag an error in the data stream if there is an LV fault to the module or if the chips become desynchronised from the ATLAS system due to single-event upsets (soft errors in the electronics). The rate of link errors was minimised by the online monitoring of chip errors in the data and the automatic reconfiguration of the modules with those errors. In addition, in 2011 an automatic global reconfiguration of all SCT module chips every 30 minutes was implemented, as a precaution against subtle deterioration in chip configurations as a result of single-event upsets. Figure 7 shows the fraction of links giving errors in physics runs during 2012 data-taking; the rate of such errors was generally very low despite the ROD data-processing issues which artificially increased the rate of such errors during periods with high trigger rates and high occupancy levels.

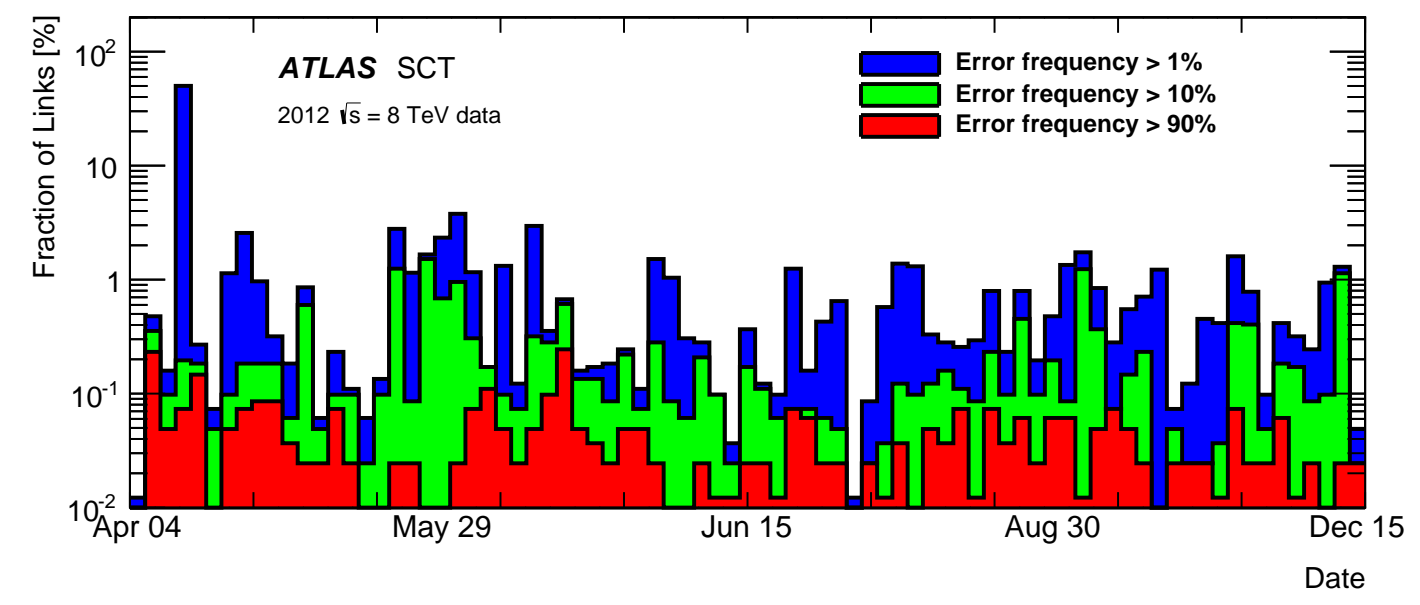

Figure 7. Fraction of data links giving errors during 2012 data-taking.

\subsection{Operations issues}

Operations from 2010 to 2013 were reasonably trouble-free with little need for expert intervention, other than performing regular calibrations between physics fills. However, a small number of issues needed vigilance and expert intervention on an occasional basis. Apart from the ROD-busy issues discussed above, the main actions were to monitor and adjust the leakage-current trip limits to counter the increase in leakage currents due to radiation or due to anomalous leakage-current behaviour (see section 7.5), and the replacement of failing off-detector optical transmitters.

The only significant component failure for the SCT has been the VCSEL arrays of the offdetector TX optical transmitters (see section 2.2). The immediate consequence of a TX channel failure was that the module no longer received the clock and command signals, and therefore no longer returned data. VCSEL failures were originally attributed to inadequate electrostatic discharge (ESD) precautions during the assembly of the VCSEL arrays into the TX plug-in, and the 
entire operational stock of 364 of the 12-channel TXs were replaced in 2009 with units manufactured with improved ESD procedures. Although this resulted in a small improvement in lifetime, further TX channel failures continued at a rate of 10-12 per week. These failures were finally attributed to degradation arising from exposure of the VCSELs to humidity. In 2012, new VCSEL arrays were installed; these VCSELs contained a dielectric layer to act as a moisture barrier. As expected these TXs demonstrated improved robustness against humidity, compared to the older arrays, when measuring the optical spectra of the VCSEL light in controlled conditions [23]. During 2012, the new VCSELs nonetheless had a small but significant failure rate, suspected to be a result of mechanical stress arising from thermal mismatch between the optical epoxy on the VCSEL surface and the GaAs of the VCSEL itself. This was despite the introduction of dry air to the ROD racks in 2012, which reduced the relative humidity levels from $\sim 50 \%$ in 2010 to $\sim 30 \%$. For future ATLAS runs, commercially available VCSELs inside optical sub-assemblies, with proven robustness and reliability, and packaged appropriately on a TX plug-in for the BOC, will be installed.

Operationally, the impact of the TX failures was minimised by the use of the TX redundancy mechanism, which could be applied during breaks between physics fills. If this was not available (for example, if the module providing the redundancy was also using the redundancy mechanism itself) then the module was disabled until the opportunity arose to replace the TX plug-in on the BOC. As a consequence, the number of disabled modules fluctuated slightly throughout the years, but rarely increased by more than 5-10 modules.

The RX data links have been significantly more reliable than the TX links, which is attributed to the use of a different VCSEL technology ${ }^{7}$ and the fact that the on-detector VCSELs operate at near-zero humidity. There have been nine confirmed failures of on-detector VCSEL channels since the beginning of SCT operations. The RX lifetime will be closely monitored over the coming years; replacements of failed RX VCSELs will not be possible due to the inaccessibility of the detector, so RX redundancy will remain the only option for RX failures.

\section{Offline reconstruction and simulation}

\subsection{Track reconstruction}

Tracks are reconstructed using ATLAS software within the Athena framework [24]. Most studies in this paper use tracks reconstructed from the whole inner detector, i.e. including pixel detector, SCT and TRT hits, using the reconstruction algorithms described in ref. [25]. For some specialised studies, tracks are reconstructed from SCT hits alone.

The raw data from each detector are first decoded and checked for error conditions. Groups of contiguous SCT strips with a hit are grouped into a cluster. Channels which are noisy, as determined from either the online calibration data or offline monitoring (see section 5.4), or which have other identified problems, are rejected at this stage. It is also possible to select or reject strips with specific hit patterns in the three time bins, for example to study the effect of requiring an X1X hit pattern (see section 2.2) on data taken in any-hit mode. The one-dimensional clusters from the two sides of a module are combined into three-dimensional space-points using knowledge of the stereo angle and the radial (longitudinal) positions of the barrel (endcap) modules.

\footnotetext{
${ }^{7}$ These VCSELs used the older proton-implant technology for current channelling. This technology is more reliable but has become obsolete and has been replaced by oxide-implant VCSELs.
} 
Pixel clusters are formed from groups of contiguous pixels with hits, in a fashion similar to clusters of SCT strips. In this case, knowledge of the position of a single pixel cluster is enough to construct a space-point. The three-dimensional space-points in the pixel detector and the SCT, together with drift circles in the TRT, form the input to the pattern recognition algorithms.

Track seeds are formed from sets of three space-points in the silicon detectors; each spacepoint must originate in a different layer. The seeds are used to define roads, and further silicon clusters within these roads are added to form track candidates. Clusters can be attached to multiple track candidates. An ambiguity-resolving algorithm is used to reject poor track candidates until hits are assigned to only the most promising one. A track fit is performed to the clusters associated to each track. Finally, the track is extended into the TRT by adding drift circles consistent with the track extension, and a final combined fit is performed [26].

The reconstruction of SCT-only tracks is similar, but only SCT space-points are used in the initial seeds, and only SCT clusters in the final track fit.

The average number of SCT clusters per track is around eight in the barrel region $(|\eta| \lesssim$ 1) and around nine in the endcap regions, as shown in figure 8 for a sample of minimum-bias events recorded at $\sqrt{s}=8 \mathrm{TeV}$. In this figure, data are compared with simulated minimum-bias events generated using PYTHIA8 [27] with the A2:MSTW2008LO tune [28]. The simulation is reweighted such that the vertex $z$ position and track transverse momentum distributions match those observed in the data. The tracks are required to have at least six hits in the SCT and at least two hits in the pixel detector, transverse $\left(d_{0}\right)$ and longitudinal $\left(z_{0}\right)$ impact parameters with respect to the primary vertex of $\left|d_{0}\right|<1.5 \mathrm{~mm}$ and $\left|z_{0} \sin \theta\right|<4 \mathrm{~mm}$ respectively, and a minimum transverse momentum of $100 \mathrm{MeV}$. The variation with pseudorapidity of the mean number of hits arises primarily from the detector geometry. The offset of the mean primary-vertex position in $z$ with respect to the centre of the detector gives rise to the small asymmetry seen in the barrel region. The variation in mean number of hits with pseudorapidity is well reproduced by the simulation.

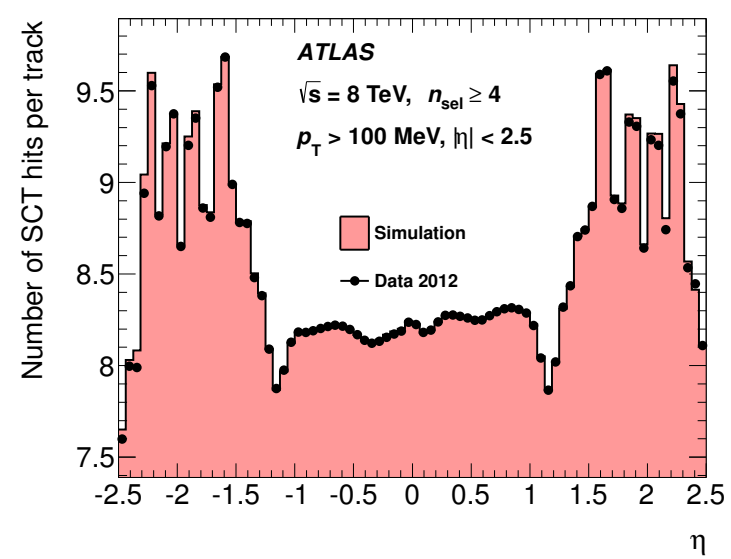

Figure 8. Comparison between data (dots) and simulation (histogram) of the average number of SCT clusters (hits) per track as a function of pseudorapidity, $\eta$, measured in minimum-bias events at $\sqrt{s}=8 \mathrm{TeV}$. 


\subsection{Track alignment}

Good knowledge of the alignment of the inner detector is critical in obtaining the optimal tracking performance. The design requirement is that the resolution of track parameters be degraded by no more than $20 \%$ with respect to the intrinsic resolution [29], which means that the SCT modules must be aligned with a precision of $12 \mu \mathrm{m}$ in the direction perpendicular to the strips. The principle method of determining the inner detector alignment uses a $\chi^{2}$ technique that minimises the residuals to fitted tracks from $p p$ collision events. Alignment is performed sequentially at different levels of detector granularity starting with the largest structures (barrel, endcaps) followed by alignment of individual layers and finally the positions of individual modules are optimised. The number of degrees of freedom at the different levels increases from 24 at the first level to 23328 at the module level. This kind of alignment addresses the 'strong modes', where a misalignment would change the $\chi^{2}$ distribution of the residuals to a track. There is also a class of misalignment where the $\chi^{2}$ fit to a single track is not affected but the value of measured momentum is systematically shifted. These are the 'weak-mode' misalignments which are measured using a variety of techniques, for example using tracks from the decay products of resonances like the $J / \psi$ and the $Z$ boson. Details of the alignment techniques used in the ATLAS experiment can be found elsewhere [30, 31].

The performance of the alignment procedure for the SCT modules is validated using high- $p_{\mathrm{T}}$ tracks in $Z \rightarrow \mu \mu$ events in $p p$ collision data at $\sqrt{s}=8 \mathrm{TeV}$ collected in 2012. Figure 9 shows residual distributions in $x$ (i.e. perpendicular to the strip direction) for one representative barrel and one endcap disk, compared with the corresponding distributions from simulations with an ideal geometry. The good agreement between the data and simulation in the measured widths indicates that the single plane position resolution of the SCT after alignment is very close to the design value of $17 \mu \mathrm{m}$ in $r-\phi$. Similar good agreement is seen in all other layers. From a study of the residual distances of the second-nearest cluster to a track in each traversed module, the two-particle resolution is estimated to be $\sim 120 \mu \mathrm{m}$.

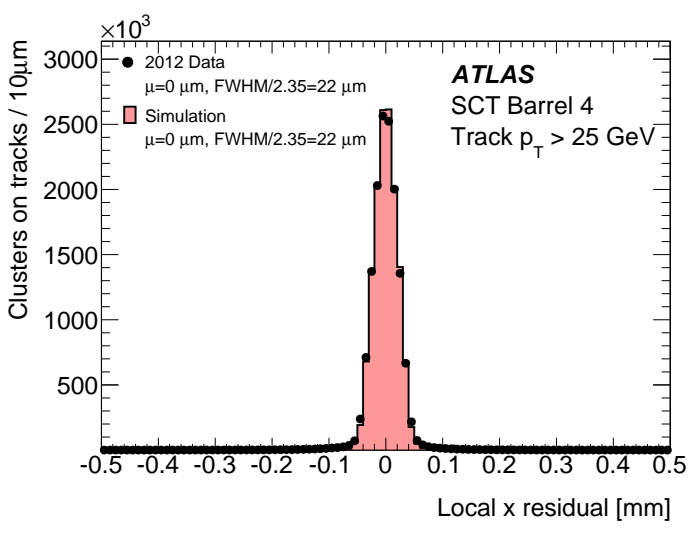

(a)

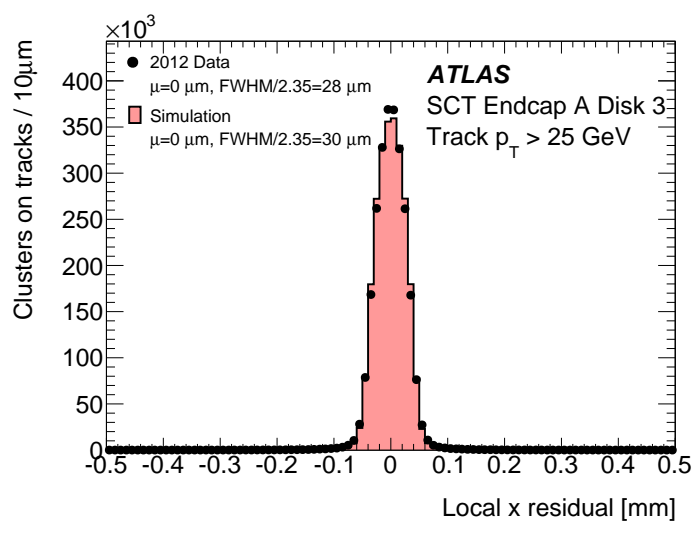

(b)

Figure 9. Distributions of residuals measured in $Z \rightarrow \mu \mu$ events at $\sqrt{s}=8 \mathrm{TeV}$ for data (points) compared with simulation (histogram), for (a) barrel 4 and (b) disk 3 of endcap A. 


\subsection{Simulation}

Most physics measurements performed by the ATLAS collaboration rely on Monte Carlo simulations, for example to calculate acceptances or efficiencies, to optimise cuts in searches for new physics phenomena or to understand the performance of the detector. Accurate simulation of the detector is therefore of great importance. Simulation of the SCT is carried out using GEANT4 [32] within the ATLAS simulation framework [33]. A detailed model of the SCT detector geometry is incorporated in the simulation program. The silicon wafers are modelled with a uniform thickness of $285 \mu \mathrm{m}$ and planar geometry; the distortions measured in the real modules [7] are small and therefore not simulated. Modules can be displaced from their nominal positions to reflect those measured in the track alignment procedure in data. All services, support structures and other inert material are included in the simulation. The total mass of the SCT in simulation matches the best estimate of that of the real detector, which is known to a precision of better than 5\%.

Propagation of charged particles through the detector is performed by GEANT4. An important parameter is the range cut, above which secondary particles are produced and tracked. In the silicon wafers this cut is set to $50 \mu \mathrm{m}$, which corresponds to kinetic energies of $80 \mathrm{keV}, 79 \mathrm{keV}$ and $1.7 \mathrm{keV}$ for electrons, positrons and photons, respectively. During the tracking, the position and energy deposition of each charged particle traversing a silicon wafer are stored. These energy deposits are converted into strip hits in a process known as digitisation, described below. The simulated data are reconstructed using the same software as used for real data. Inoperative modules, chips and strips are simulated to match average data conditions.

\subsubsection{Digitisation model}

The digitisation model starts by converting the energy deposited in each charged-particle tracking step in the silicon into a charge on the readout electrodes. Each tracking step, which may be the full width of the wafer, is split into $5 \mu \mathrm{m}$ sub-steps, and the energy deposited shared uniformly among these sub-steps. The energy is converted to charge using the mean electron-hole pair-creation energy of $3.63 \mathrm{eV} /$ pair, and the hole charge is drifted to the wafer readout surface in a single step taking into account the Lorentz angle, $\theta_{\mathrm{L}}$, and diffusion. The Lorentz angle is the angle between the drift direction and the normal to the plane of the sensor which arises when the charge carriers are subject to the magnetic field from the solenoid as well as the electric field generated by the bias voltage. A single value of the Lorentz angle, calculated as described in section 6.5 assuming a uniform value of the electric field over the entire depth of the wafer, is used irrespective of the original position of the hole cloud. The drift time is calculated as the sum of two components: one corresponding to drift perpendicular to the detector surface, calculated assuming an electric field distribution as in a uniform flat diode, and a second corresponding to drift along the surface, introduced to address deficiencies in the simple model and give better agreement with test-beam data.

The second step in the digitisation process is the simulation of the electronics response. For each charge arriving at a readout strip, the amplifier response at three times, corresponding to the three detector readout bins, is calculated. The cross-talk signal excited in each neighbouring strip, which is a differential form of the main strip pulse, is also calculated. Electronic noise is added to the strips with charge, generated from a Gaussian distribution with mean zero and standard deviation equal to the equivalent noise charge taken from data. The noise is generated 
independently for each time bin. Finally, strips with a signal above the readout threshold, normally $1 \mathrm{fC}$, are recorded. Further random strips from among those without any charge deposited are added to the list of those read out, to reproduce the noise occupancy observed in data.

\subsubsection{Induced-charge model}

In order to understand better the performance of the detector, and to check the predictions of the simple digitisation model, a full, but time-consuming, calculation was used. In this 'inducedcharge model' the drift of both electrons and holes in the silicon is traced step-by-step from the point of production to the strips or HV plane, and the charge induced on the strips from this motion calculated using a weighting potential according to Ramo's theorem [34, 35].

The electron and hole trajectories are split into steps with corresponding time duration $\delta t=$ $0.1 \mathrm{~ns}$ or $0.25 \mathrm{~ns}$. The magnitude of the drift velocity $v_{\mathrm{d}}$ at each step is calculated as:

$$
v_{\mathrm{d}}=\mu_{\mathrm{d}} E
$$

where $\mu_{\mathrm{d}}$ is the mobility and $E$ is the magnitude of the electric field strength. The effect of diffusion is included by choosing the actual step length, independently in each of two perpendicular distributions, from a Gaussian distribution of width $\sigma$ given by:

$$
\sigma=\sqrt{2 D \delta t} ; \quad \text { with } \quad D=k_{\mathrm{B}} T \mu_{\mathrm{d}} / e
$$

where the diffusion coefficient $D$ depends on the temperature $T ; k_{\mathrm{B}}$ is Boltzmann's constant and $-e$ the electron charge. The electric field at each point is calculated using a two-dimensional finiteelement model (FEM); the dielectric constant $\left(11.6 \epsilon_{0}\right)$ and the donor concentration in the depleted region are taken into account in the FEM calculation. In the presence of a magnetic field, the direction of drift is rotated by the local Lorentz angle.

The induced charge on a strip is calculated from the motion of the holes and electrons using a weighting potential obtained using the same two-dimensional FEM by setting the potential of one strip to $1 \mathrm{~V}$ with all the other strips and the HV plane at ground. In the calculation the space charge is set to zero since its presence does not affect the validity of Ramo's theorem [36]. The simulation of the electronics response follows the same procedure as in the default digitisation model above.

The induced-charge model predicts an earlier peaking time for the charge collected on a strip than the default model: up to $10 \mathrm{~ns}$ for charge deposited midway between strips. However, after simulation of the amplifier response and adjusting the timing to maximise the fraction of clusters with a $01 \mathrm{X}$ time-bin pattern, the output pulse shapes from the two models are similar. The mean cluster widths predicted by the induced-charge model are slightly larger than those predicted by the default digitisation model. The difference is about 0.02 strips $(1.6 \mu \mathrm{m})$ for tracks with incident angles near to the Lorentz angle. The position of the minimum cluster width occurs at incident angles about $0.1^{\circ}$ larger in the induced-charge model. Since the differences between the two models are small, the default digitisation model described in section 4.3.1 is used in the ATLAS simulation.

\subsection{Conditions data}

The offline reconstruction makes use of data describing the detector conditions in several ways. First, bad channels are rejected from cluster formation. In the pattern recognition stage, knowledge 
of dead detector elements is used in resolving ambiguities. Measured values of module bias voltage and temperature are used to calculate the Lorentz angle in the track reconstruction. In addition, measured chip gains and noise are used in the simulation. The conditions data, stored in the ATLAS COOL database [37], arise from several sources:

- Detector configuration. These data include which links are in the readout, use of the redundancy mechanism, etc., and are updated when the detector configuration is changed. They cannot be updated during a run.

- DCS. The data from the detector control system (see section 2.3) most pertinent to reconstruction are module bias voltage values and temperatures. Data from modules which are not at their nominal bias voltage value are excluded from reconstruction at the cluster-formation stage. This condition removes modules which suffer an occasional high-voltage trip, resulting in high noise occupancy, for the duration of that trip.

- Online calibration. In reconstruction, use of online calibration data is limited to removing individual strips with problems. These are mostly ones which were found to be noisy in the latest preceding calibration runs.

- Offline monitoring. Noisy strips are identified offline run-by-run, as described in section 5.4. These are also removed during cluster formation.

\section{Monitoring and data quality assessment}

Continuous monitoring of the SCT data is essential to ensure good-quality data for physics analysis. Data quality monitoring is performed both online and offline.

\subsection{Online monitoring}

The online monitoring provides immediate feedback on the condition of the SCT, allowing quick diagnosis of issues that require intervention during a run. These may include the recovery of a module or ROD that is not returning data, or occasionally a more serious problem which requires the early termination of a run and restart.

The fastest feedback is provided by monitoring the raw hit data. Although limited in scope, this monitoring allows high statistics, minimal trigger bias and fast detector feedback. The number of readout errors, strip hits and simple space-points (identified as a coincidence between hits on the two sides of a module within \pm 128 strips of each other) are monitored as a function of time and features of a run can be studied. During collisions the hit rate increases by several orders of magnitude. This monitoring is particularly useful for providing speedy feedback on the condition of the beam, and is used extensively during LHC commissioning and the warm-start procedure.

In addition to raw-data monitoring, a fraction of events is fully reconstructed online, and monitoring histograms are produced as described below for the offline case. Automatic checks are performed on the histograms $[38,39]$ and warnings and alarms issued to the shift crew ('shifters'). 


\subsection{Offline monitoring}

Offline monitoring allows the data quality to be checked in more detail, and an assessment of the suitability of the data for use in physics analyses to be made. A subsample of events is reconstructed promptly at the ATLAS Tier0 computer farm. Monitoring plots are produced as part of this reconstruction, and used to assess data quality. These plots typically integrate over a whole run, but may cover shorter periods in specific cases. Histograms used to assess the performance of the SCT include:

- Modules excluded from the readout configuration (see section 3.1).

- Modules giving readout errors. In some cases the error rates are calculated per luminosity block so that problematic periods can be determined.

- Hit efficiencies (see section 6.4). The average hit efficiency for each barrel layer and endcap disk is monitored, as well as the efficiencies of the individual modules. Localised inefficiencies can be indicative of problematic modules, whereas global inefficiencies may be due to timing problems or poor alignment constants.

- Noise occupancy calculated using two different methods. The first counts hits not included in space-points, and is only suitable for very low-multiplicity data. The second uses the ratio of the number of modules with at least one hit on one side only to the number with no hit on either side.

- Time-bin hit patterns for hits on a track (section 2.2), which gives an indication of how well the detectors are timed in to the bunch crossings.

- Tracking performance distributions. These include the number of SCT hits per track, transverse momentum, $\eta, \phi$ and impact parameter distributions; track fit residual and pull distributions.

Automatic checks are performed on these histograms using the offline data quality monitoring framework [40], and they are also reviewed by a shifter. They form the basis of the data quality assessment discussed in the next section.

\subsection{Data quality assessment}

The data quality is assessed for every run collected, and the results are stored in a database [41] by setting one or more 'defect flags' if a problem is found. Each defect flag corresponds to a particular problem, and may be set for a whole run or only a short period. Several defect flags may be set for the same period. These flags are used later to define good data for physics analysis. The SCT defect flags also provide operational feedback as to the current performance of the detector.

The fraction of the data recorded which is affected by each SCT issue is given in the upper two parts of table 4. The uppermost part of the table shows intolerable defects, which lead to the affected runs or luminosity-block ranges being excluded from physics analyses. The most common problem in this category is the exclusion of two or more RODs (96 modules, 2.3\% of the detector) or a whole crate ( $12 \%$ of the detector) from the readout, which results in a loss in 
tracking coverage in a region of the detector. The exclusion of RODs or crates affects a limited but significant region of the detector. Other intolerable defects affect the whole detector. The global reconfiguration of all readout chips occasionally causes data errors for a short period; the SCT can become desynchronised from the rest of ATLAS; occasionally data are recorded with the modules at standby voltage $(50 \mathrm{~V})$.

For more minor issues, or when the excluded modules have less of an impact on tracking coverage, a tolerable defect is set. These defects are shown in the central part of table 4. One excluded ROD is included in this category. A single excluded ROD in the endcap (the most usual case) has little impact on tracking because it generally serves modules on one disk only. A ROD in the barrel serves modules in all layers in an azimuthal sector, and thus may affect tracking performance; this is assessed as part of the global tracking performance checks. Other tolerable defects include more than 40 modules excluded from the readout or giving readout errors, which indicate detector problems but do not significantly affect tracking performance. Although noise occupancy is monitored, no defect is set for excessive noise occupancy.

In addition to SCT-specific checks, the global tracking performance of the inner detector is also evaluated, and may indicate problems which arise from the SCT but which are not flagged with SCT defects, or which are flagged as tolerable by the SCT but are intolerable when the whole inner detector is considered. This corresponds to situations where the pixel detector or TRT have a simultaneous minor problem in a similar angular region. The fraction of data affected by issues determined from global tracking performance is given in the lower part of table 4 . There is significant overlap between the SCT defects and the tracking performance defects.

The total fraction of data flagged as bad for physics in 2011 (2012) by SCT-specific checks was $0.44 \%(0.89 \%)$. A further $0.33 \%(0.56 \%)$ of data in 2011 (2012) was flagged as bad for physics by tracking performance checks but not by the SCT alone. The higher fraction in 2012 mainly resulted from RODs disabled due to high occupancy and trigger rates, as discussed in section 3.4.

\subsection{Prompt calibration loop}

Reconstruction of ATLAS data proceeds in two stages. First a fraction of the data from each run is reconstructed immediately, to allow detailed data quality and detector performance checks. This is followed by bulk reconstruction some $24-48$ hours after the end of the run. This delay allows time for updated detector calibrations to be obtained. For the SCT no offline calibrations are performed during this prompt calibration loop, but the period is used to obtain conditions data. In particular, strips that have become noisy since the last online calibration period are found and excluded from the subsequent bulk reconstruction. Other conditions data, such as dead strips or chips, are obtained for monitoring the SCT performance.

The search for noisy strips uses a special data stream triggered on empty bunch crossings, so that no collision hits should be present. Events are written to this stream at a rate of $2-10 \mathrm{~Hz}$, giving sufficient data to determine noisy strips run by run for all but the shortest runs. Processing is performed automatically on the CERN Tier0 computers and the results are uploaded to the conditions database after a check by the shifter.

A noisy strip is defined as one with an average occupancy (during the period with $\mathrm{HV}$ on) of more than $1.5 \%$. Excluding those already identified as noisy in the online calibration runs $(0.06 \%$ of strips in 2012-2013), the number of noisy strips found has increased from $\sim 100$ during 2010 to 
Table 4. The data quality defects recorded for 2011 and 2012, and percentage of the luminosity affected. The data assigned intolerable defects (marked ' $\mathrm{Y}$ ' in the table) are not included in physics analyses. Empty entries indicate that the defect was not defined for that year. Multiple defect flags may be set for the same data.

\begin{tabular}{|c|c|c|c|}
\hline \multirow[t]{2}{*}{ Defect Description } & \multirow[t]{2}{*}{$\begin{array}{c}\text { Intolerable } \\
\text { Defect }\end{array}$} & \multicolumn{2}{|c|}{$\begin{array}{l}\text { Luminosity } \\
\text { Affected [\%] }\end{array}$} \\
\hline & & 2011 & 2012 \\
\hline \multicolumn{4}{|l|}{ SCT intolerable defects } \\
\hline Crate(s) excluded from readout & Y & 0.23 & 0.07 \\
\hline Two or more RODs excluded from readout & Y & 0.15 & 0.45 \\
\hline SCT global desynchronisation & Y & 0.04 & 0.00 \\
\hline SCT bias voltage at standby $(50 \mathrm{~V})$ & $\mathrm{Y}$ & 0.02 & 0.07 \\
\hline Readout errors after global reconfiguration & Y & - & 0.30 \\
\hline \multicolumn{4}{|l|}{ SCT tolerable defects } \\
\hline Less than $99 \%$ average efficiency in one of barrel or endcap regions & $\mathrm{N}$ & 8.75 & 8.33 \\
\hline SCT not at standard bias voltage & $\mathrm{N}$ & 1.64 & 0.05 \\
\hline More than 40 modules with errors in short period of time & $\mathrm{N}$ & 1.13 & 0.43 \\
\hline Exactly one ROD excluded from readout & $\mathrm{N}$ & 0.93 & 2.90 \\
\hline More than 40 modules with errors & $\mathrm{N}$ & 0.74 & 1.88 \\
\hline Non-standard timing, e.g. timing scans & $\mathrm{N}$ & 0.06 & 0.21 \\
\hline More than 40 modules excluded & $\mathrm{N}$ & - & 0.82 \\
\hline Too few events for DQ judgement & $\mathrm{N}$ & 0.00 & 0.05 \\
\hline \multicolumn{4}{|l|}{ Tracking performance defects } \\
\hline Global tracking performance is impacted by $\mathrm{SCT}$ issue & $\mathrm{N}$ & 1.09 & 2.09 \\
\hline Significant loss of tracking coverage & $\mathrm{Y}$ & 0.71 & 0.94 \\
\hline SCT impacting $b$-tagging performance & $\mathrm{N}$ & - & 0.35 \\
\hline SCT seriously impacting $b$-tagging performance & Y & - & 0.10 \\
\hline
\end{tabular}

a few thousand during 2012, as shown in figure 10. Significant fluctuations are observed from run to run. These arise from two sources: single-event upsets causing whole chips to become noisy and the abnormal leakage-current increases observed in some endcap modules (see section 7). Both of these effects depend on the instantaneous luminosity, and thus vary from run to run. The maximum number of noisy strips, observed in a few runs, corresponds to $<0.25 \%$ of the total number of strips in the detector.

\section{Performance}

\subsection{Detector occupancy}

The design of the SCT was optimised to have low detector occupancy to reduce confusion in pattern recognition that arises in high-multiplicity final states resulting from multiple proton-proton interactions. For the initial design luminosity of $10^{34} \mathrm{~cm}^{-2} \mathrm{~s}^{-1}$ at a beam-crossing rate of $40 \mathrm{MHz}$ the mean number of interactions per crossing was expected to be 23 . In these circumstances, the 


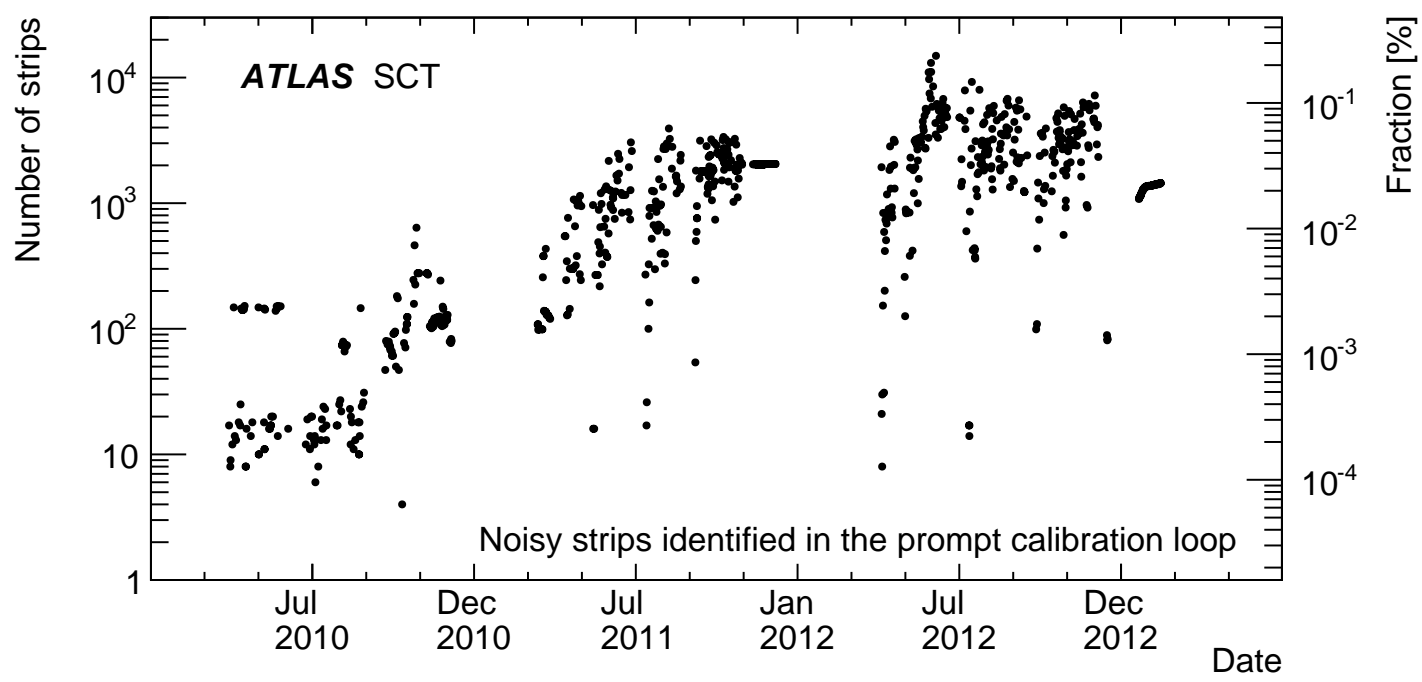

Figure 10. Number of noisy strips (also shown as the fraction of all strips on the right-hand axis) found in the prompt calibration loop for physics runs with greater than one hour of stable beams during 2010-2013. Noisy strips identified in online calibration data are excluded from the total.

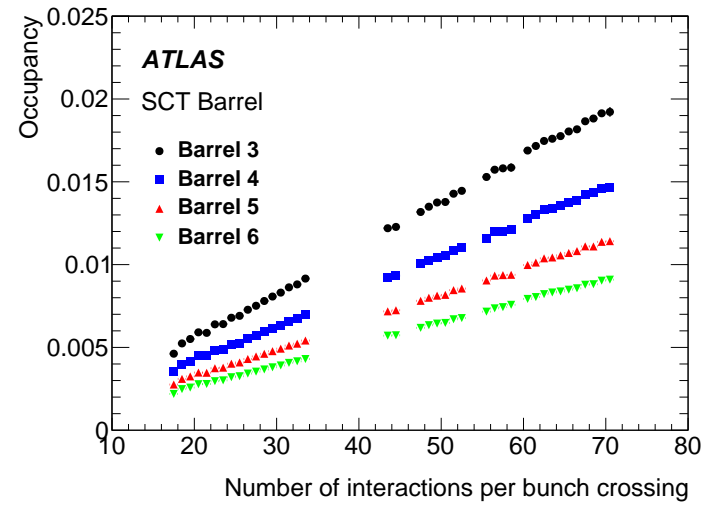

(a)

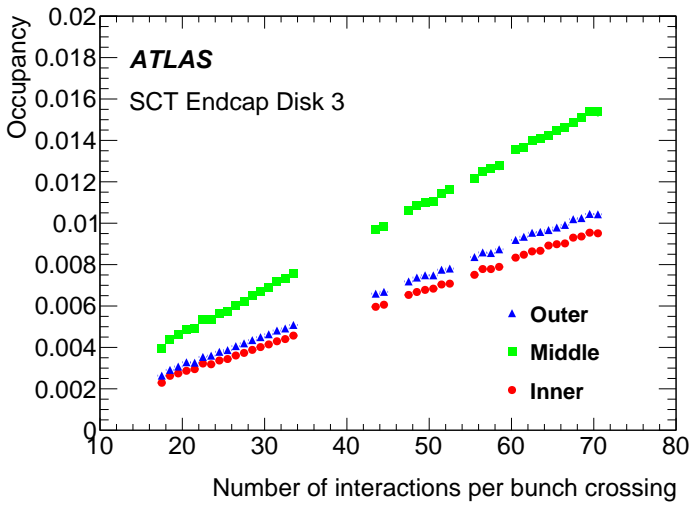

(b)

Figure 11. Mean occupancy of (a) each barrel and (b) inner, middle and outer modules of endcap disk 3 as a function of the number of interactions per bunch crossing in minimum-bias $p p$ data. Values for less than 40 interactions per bunch crossing are from collisions at $\sqrt{s}=7 \mathrm{TeV}$, those for higher values from collisions at $\sqrt{s}=8 \mathrm{TeV}$, which have an occupancy larger by a factor of about 1.03 for the same number of interactions per bunch crossing.

mean strip occupancy was expected to be less than 1\%. In 2012 with an LHC bunch-spacing of $50 \mathrm{~ns}$ the design goals for pile-up were exceeded with no significant loss of tracking efficiency.

In figure 11 the occupancy, defined as the number of strips above threshold divided by the total number of strips, is shown for the four barrels and for the different module types in one representative endcap disk (averaging over both endcaps). The SCT was read out in level mode, i.e. demanding a hit in the in-time bunch crossing, and noisy strips identified in online calibration runs 


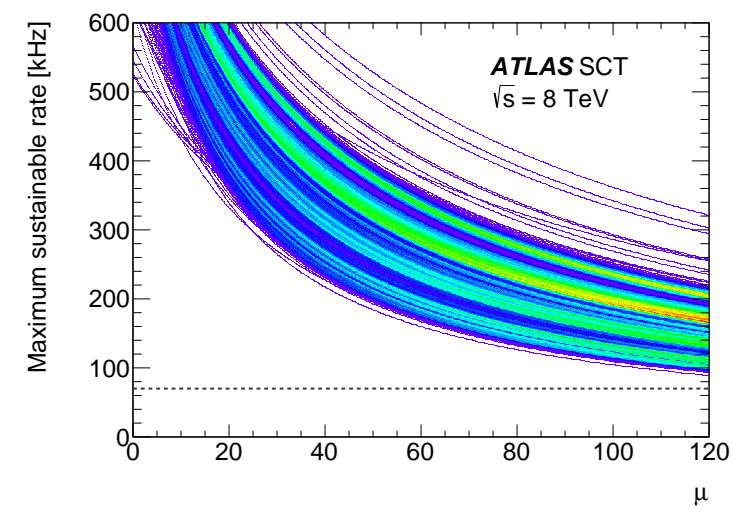

(a)

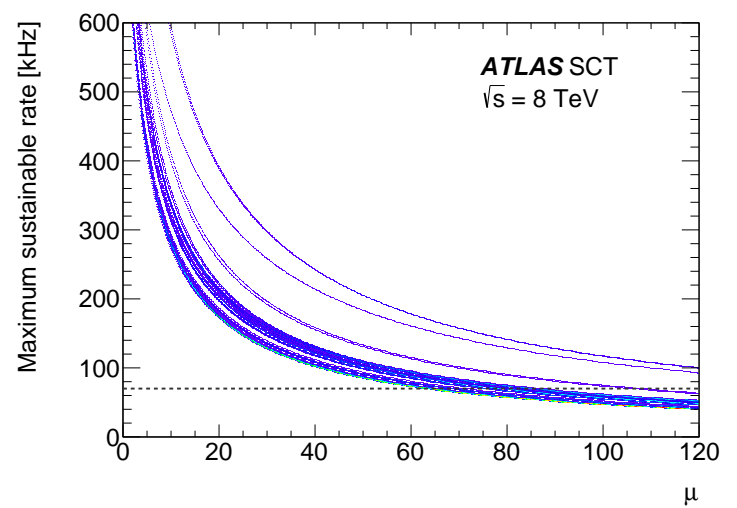

(b)

Figure 12. Maximum sustainable level-1 trigger rate for the transfer of data from (a) front-end chip to ROD and (b) ROD to ATLAS DAQ chain, as a function of the mean number of interactions per bunch crossing, $\mu$. Values are calculated from measured event sizes in $p p$ interactions at $\sqrt{s}=8 \mathrm{TeV}$ assuming $90 \%$ of the available data-link bandwidth is used. Each link is shown as a separate curve. Increasing numbers of superimposed curves are indicated by changes of colour from blue through green and yellow to red. The dashed lines indicate the typical 2012 trigger rate of $70 \mathrm{kHz}$.

or the prompt calibration loop were removed. The data were recorded using a minimum-bias trigger in special high pile-up runs, where average numbers of interactions per bunch crossing greater than the 35 routinely seen in physics runs could be achieved, and events were required to have at least one reconstructed primary vertex. Values for less than 40 interactions per bunch crossing are from collisions at $\sqrt{s}=7 \mathrm{TeV}$, those for higher values from collisions at $\sqrt{s}=8 \mathrm{TeV}$. The occupancy at $8 \mathrm{TeV}$ is expected to be a factor of about 1.03 larger than at $7 \mathrm{TeV}$ for the same number of interactions per bunch crossing. Allowing for this, good linearity is observed up to 70 interactions per crossing, where the average occupancy is less than $2 \%$ in the innermost barrel and $\sim 1.5 \%$ in the middle modules of the central disks (the middle modules have the largest pseudorapidity coverage and hence largest occupancy in the endcaps).

The readout links were designed to accommodate up to $2 \%$ occupancy at a level- 1 trigger rate of $100 \mathrm{kHz}$ without imposing dead-time. If the trigger rate and/or occupancy is significantly increased beyond these limits, the first two limiting bottlenecks within the SCT DAQ are the bandwidth of the data links between the modules and the BOCs, and the bandwidth of the data fibres (S-links) that connect the RODs and BOCs to the ATLAS DAQ chain. The data volumes in these links were studied in $p p$ collision data at $\sqrt{s}=8 \mathrm{TeV}$ to identify any potential problems. The studies used the data stream that is reconstructed immediately for data quality assessment. The detector occupancy in this stream, which comprises a mixture of triggers, is a few percent higher than in minimum-bias events. The measured data size for each of the optical links was used to calculate the maximum sustainable rate of level-1 triggers as a function of the mean number of inelastic $p p$ interactions per bunch crossing, $\mu$. The maximum sustainable rate assumes $90 \%$ of the data-link bandwidth is used. The results for each of the 8176 links from front-end chip to ROD, excluding the small number reading out both module sides, are shown in figure 12a. Figure 12b shows a similar distribution for the transfer of data on the 90 S-links. It can be seen from these plots that 
the slowest data links are still adequate up to a pile-up, $\mu$, of 120, while the S-links impose a limit of $\mu<62$ at the typical 2012 trigger rate of $70 \mathrm{kHz}$.

Significant increases in trigger rate and pile-up are anticipated with the increase in instantaneous luminosities foreseen at the LHC beyond 2015 at $\sqrt{s}=14 \mathrm{TeV}$. The increase in multiplicity and trigger rate will impose a reduced limit on $\mu$ of $\sim 87$ and $\sim 33$ at $100 \mathrm{kHz}$ for the data links and S-links respectively, if there are no changes made to the existing DAQ. The limit from the data links arises from components mounted on the detector, and therefore cannot be improved. The limit from the S-links will be improved by increasing the number of RODs, BOCs and S-links from 90 to 128; the load per S-link will therefore be reduced and balanced in a more equal way. In addition, edge-mode readout (01X, see section 2.2)) will be deployed to minimise the effect of hits from one bunch crossing appearing in another, and an improved data-compression algorithm will be deployed in the ROD. As a result of these changes, the slowest S-link will be able to sustain a level-1 trigger rate of $100 \mathrm{kHz}$ at $\mu=87$, which matches the hard limit from the front-end data links. This corresponds to a luminosity of $3.2 \times 10^{34} \mathrm{~cm}^{-2} \mathrm{~s}^{-1}$ with $25 \mathrm{~ns}$ bunch spacing and 2808 bunches per beam.

\subsection{Noise}

One of the crucial conditions for maintaining high tracking efficiency is to keep the read-out thresholds as low as possible. This is only possible when the input noise level is kept low. There are two ways of measuring the input equivalent noise charge (ENC, in units of number of electrons) in dedicated calibration runs:

1. Response-curve noise: in three-point gain-calibration runs, a threshold scan is performed with a known charge injected into each channel, as described in section 3.2. The Gaussian spread of the output noise value at the injection charge of $2 \mathrm{fC}$ (chosen because this value is well clear of non-linearities which can appear below $1 \mathrm{fC}$ ) is obtained by fitting the threshold curve. The ENC is calculated by dividing the output noise by the calibration gain of the front-end amplifier.

2. Noise Occupancy (NO): in the noise-occupancy calibration runs, a threshold scan with no charge injection is performed to obtain the occupancy as a function of threshold charge. The ENC is estimated from a linear fit to the dependence on threshold charge of the logarithm of noise occupancy.

The former value reflects the width of the Gaussian-like noise distribution around $\pm 1 \sigma$ while the latter is a measure of the noise tail at around $+4 \sigma$, being sensitive to external sources such as common-mode noise. Whenever the corresponding calibration runs are performed, the mean values of the response-curve ENC or NO at $1 \mathrm{fC}$ for each chip are saved for later analyses. These two noise estimates correlate fairly well chip-by-chip, but ENC values deduced from noise occupancies are sytematically smaller by about 5\% [42]. Below, unless specified, 'noise' refers to the ENC value from the response-curve width, and is measured for modules with bias voltage at the normal operating value of $150 \mathrm{~V}$.

Figure 13 shows the distributions of chip-averaged response-curve ENC values and noise occupancies at $1 \mathrm{fC}$ as of October 2010 and December 2012 for different module types. For the barrels, 
only modules with $<111>$ sensors are plotted; barrel 6 , which has a higher temperature and thus higher noise, is shown separately from the other three barrels. For the endcaps, chips reading out Hamamatsu and CiS sensors are shown separately. Clusters around 1000 electrons in figure 13a reflect the shorter strip lengths of endcap inner and middle-short sensors. Noise occupancies of endcap inner and middle-short modules are not shown due to large errors on the low occupancy values. Almost all chips satisfy the SCT requirement of less than $5 \times 10^{-4}$ in noise occupancy for both periods.

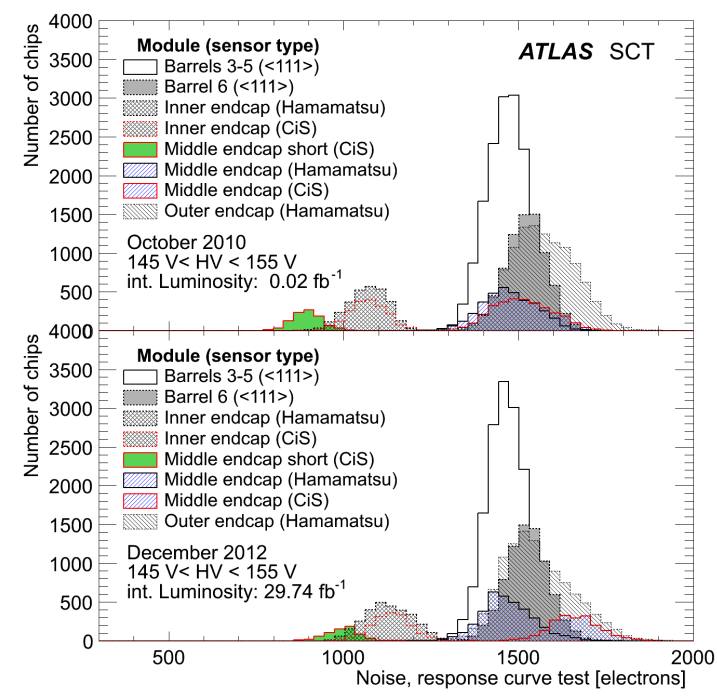

(a)

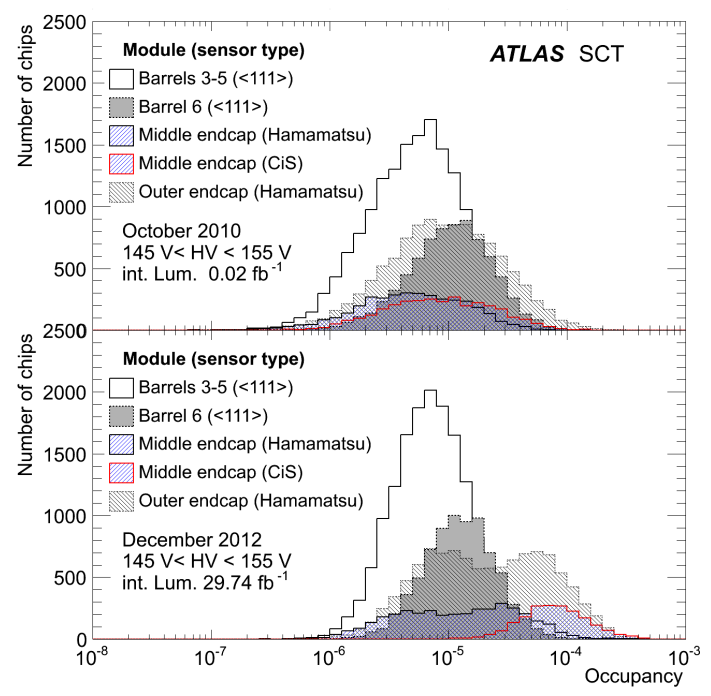

(b)

Figure 13. Distributions of chip-averaged (a) ENC from response-curve tests and (b) noise occupancy at $1 \mathrm{fC}$ from calibration runs as of October 2010 (top) and December 2012 (bottom) for different types of module.

Table 5 shows mean values of the response-curve ENCs as well as logarithmic means of the noise occupancy at $1 \mathrm{fC}$ for all ten types of module. The last column of the table shows the ratios of mean ENC values in December 2012 to those in October 2010, corrected (by at most $0.3 \%$ ) to compensate for the effects of small differences in module temperatures between the two periods. After receiving $30 \mathrm{fb}^{-1}$ of delivered luminosity, the noise remained almost unchanged for barrel and endcap outer modules, while it increased by about $10 \%$ in the endcap middle modules with CiS sensors and $6 \%$ in endcap inner modules with either Hamamatsu or CiS sensors. The median charge deposited by a minimum-ionising charged particle at normal incidence, measured in threshold scans in beam tests, is $3.5 \pm 0.1 \mathrm{fC}$ at a bias voltage of $300 \mathrm{~V}(0.15 \mathrm{fC}$ lower at $150 \mathrm{~V})$ for unirradiated modules [43]. Thus the signal-to-noise ratio is about 13 for sensors of length $12 \mathrm{~cm}$ and about 20 for endcap inner sensors. Even with the observed increase of $\sim 10 \%$ for some endcap sensors, the signal-to-noise ratio is safely higher than the value of nine needed for efficient operation. 
Table 5. Mean values of ENC from response-curve tests and noise occupancies (NO) at $1 \mathrm{fC}$, obtained in calibration runs in October 2010 and December 2012. Values are shown for each module type, separated according to sensor manufacturer or crystal orientation. The mean temperatures measured by thermistors mounted on the hybrid circuits of each module were around $2.5^{\circ} \mathrm{C}$ for barrels $3-5,10^{\circ} \mathrm{C}$ for barrel 6 and $8^{\circ} \mathrm{C}$ for the endcaps, and varied by less than one degree between October 2010 and December 2012. The last column shows ratios of ENC values measured in 2012 to those measured in 2010 corrected for the small temperature differences. Uncertainties in the ratios are less than $1 \%$.

\begin{tabular}{|lclccccc|}
\hline \multirow{2}{*}{ Module } & \multirow{2}{*}{ Type } & Manufacturer & \multicolumn{2}{c}{ October 2010 } & \multicolumn{2}{c|}{ December 2012 } & $\begin{array}{c}\langle\text { ENC }\rangle_{2012} \\
\end{array}$ \\
& 111 & Hamamatsu & 1470 & $7.5 \cdot 10^{-6}$ & 1468 & $9.0 \cdot 10^{-6}$ & 0.997 \\
\multirow{2}{*}{ Barrels 3-5 } & 100 & Hamamatsu & 1362 & $1.6 \cdot 10^{-6}$ & 1394 & $3.1 \cdot 10^{-6}$ & 1.021 \\
\hline \multirow{2}{*}{ Barrel 6 } & 111 & Hamamatsu & 1521 & $1.3 \cdot 10^{-5}$ & 1524 & $1.5 \cdot 10^{-5}$ & 1.001 \\
& 100 & Hamamatsu & 1396 & $2.4 \cdot 10^{-6}$ & 1436 & $4.8 \cdot 10^{-6}$ & 1.028 \\
\hline \multirow{2}{*}{ EC inner } & 111 & Hamamatsu & 1069 & - & 1129 & - & 1.058 \\
& 111 & CiS & 1070 & - & 1136 & - & 1.065 \\
\hline \multirow{2}{*}{ EC middle } & 111 & Hamamatsu & 1472 & $8.0 \cdot 10^{-6}$ & 1469 & $2.1 \cdot 10^{-5}$ & 1.000 \\
\multirow{2}{*}{ middle short } & 111 & CiS & 1513 & $1.4 \cdot 10^{-5}$ & 1662 & $9.5 \cdot 10^{-5}$ & 1.102 \\
EC outer & 111 & CiS & 895 & - & 1004 & - & 1.124 \\
\hline
\end{tabular}

The evolution of the response-curve noise as well as the front-end gains obtained in calibration runs from 2010 to 2012 is shown in figure 14; modules are divided into ten different groups as in table 5. All the gains are rather stable except the gradual and universal change of a few percent in mid 2011 and early 2012, the reason for which is not clear.

As can be seen in figure 14, the noise decreased by about $7 \%$ in late 2010 when the fluence level at the modules was around $10^{10} \mathrm{~cm}^{-2}$, a dose level of a few Gy. The noise drop varied strongly with the strip location as shown by the chip-number dependence in figure 15 for barrel 5 as an example. It occurred systematically in all modules except those with $<100>$ sensors. In addition, the decrease occurred first in barrel 3 and later in barrel 6 indicating fluence-dependent effects. Similar noise decreases were observed in the endcap modules but they strongly depended on the module side. Disconnected strip channels showed no decrease. In 2011 and 2012, no such noise decrease was observed, rather the noise returned to the original value and the chip-number dependence disappeared as shown by the 2011 points in figure 15 . A beam test at the CERNPS with a low rate reproduced qualitatively such a trend at a similar dose level. This unexpected phenomenon is probably caused by charge accumulated on the sensor surface leading to effective capacitance changes.

\subsection{Alignment stability}

While the alignment is determined using tracks (as described in section 4.2), the FSI system is used to confirm the observation of movement in the SCT.

Figure 16 shows the variation of track-based alignment translations for the largest structures during the $p p$ collision data-taking period from May to June 2011. The corrections shown are for translations in the global $x$ direction. Movements of the detector are measured after hardware 


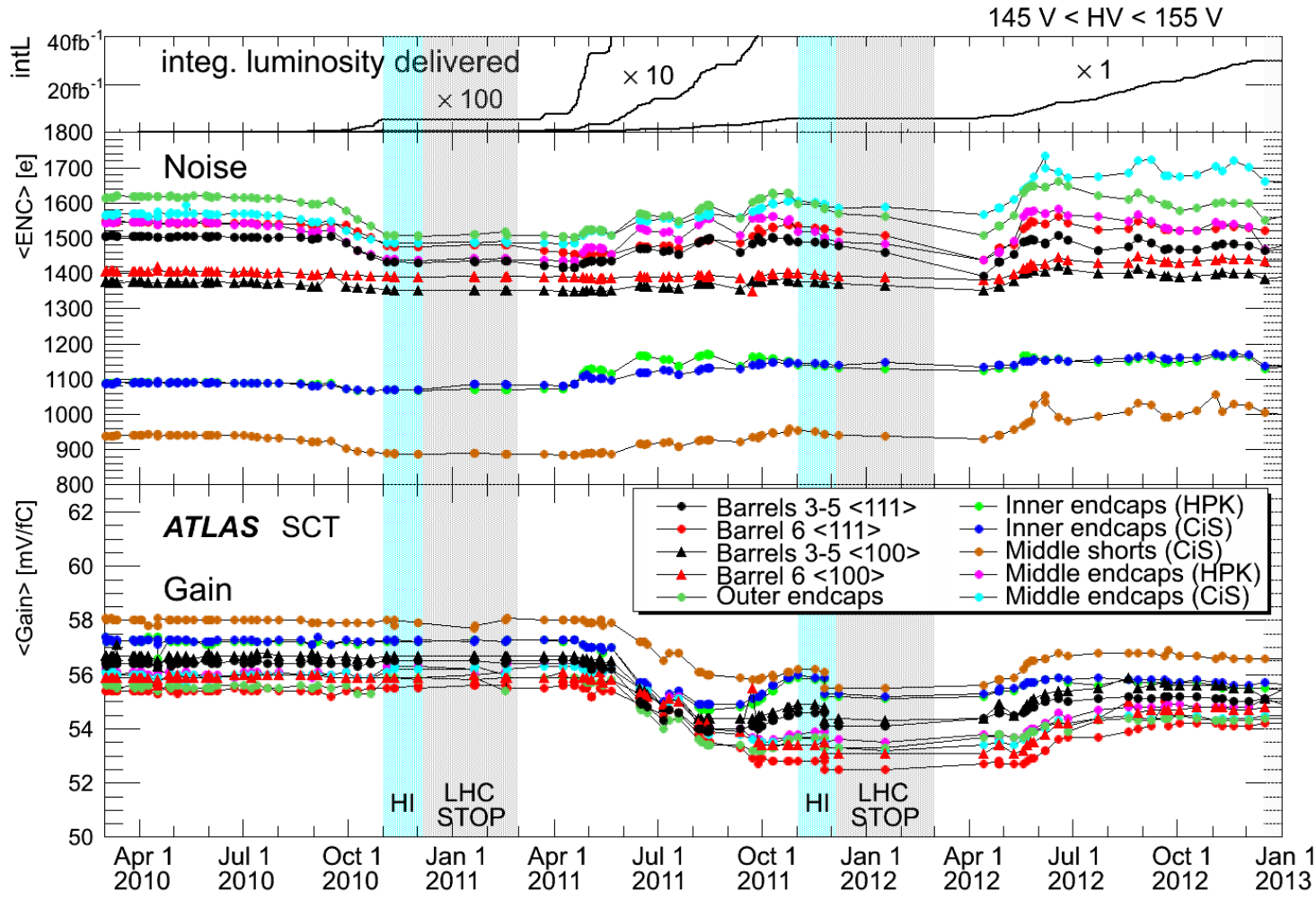

Figure 14. Integrated luminosity (top), noise (middle) and calibration gains of front-end amplifiers (bottom) as a function of time. Modules are divided into ten different groups according to their module type, crystal orientation $<111>$ vs $<100>$ and sensor manufacturer (Hamamatsu is labelled HPK). The blue shading and label 'HI' indicate periods of heavy-ion running, while extended periods with no beam in the LHC during which the SCT was off are shaded grey.

incidents such as toroid-magnet ramping as indicated in the first bin of figure 16. In between these periods little $(<1 \mu \mathrm{m})$ movement is observed indicating that the detector is generally very stable.

The most important result from FSI measurements is that the SCT detector is found to be stable at the $\mu \mathrm{m}$ level over extended periods of time, in agreement with the track-based alignment results shown in figure 16. Figure 17 shows the SCT barrel-flange movement for a subset of grid lines during a 72-hour period in May 2011. The lower plot shows the instantaneous luminosity during this period. In the upper plot, the top three curves show the measurements from outer barrel-flange lines (shifted by $+3 \mu \mathrm{m}$ ), the three curves in the middle show the measurements from the middle flange lines and the bottom three curves (shifted by $-3 \mu \mathrm{m}$ ) show the measurements from the inner flange lines. The colours correspond to those in figure $4 \mathrm{c}$. The changes in the lengths of the flange lines (movements) are most likely correlated to changes in power load (and hence temperature) in the front-end amplifiers at high trigger rate, and indicate a radial expansion and relative rotation of the barrels. The scale of the deviation is at the $\mu \mathrm{m}$ level and when the heating is removed the detector returns to the same pre- $p p$-collision position. The very gradual trend observed in some lines in figure 17 is due to the drift of the lasers over time, and does not reflect a physical effect on the grid-line length. 
Chip No. $\quad 1 \quad 2 \quad 3 \quad 4 \quad 5 \quad 6 \quad$ (link-0)
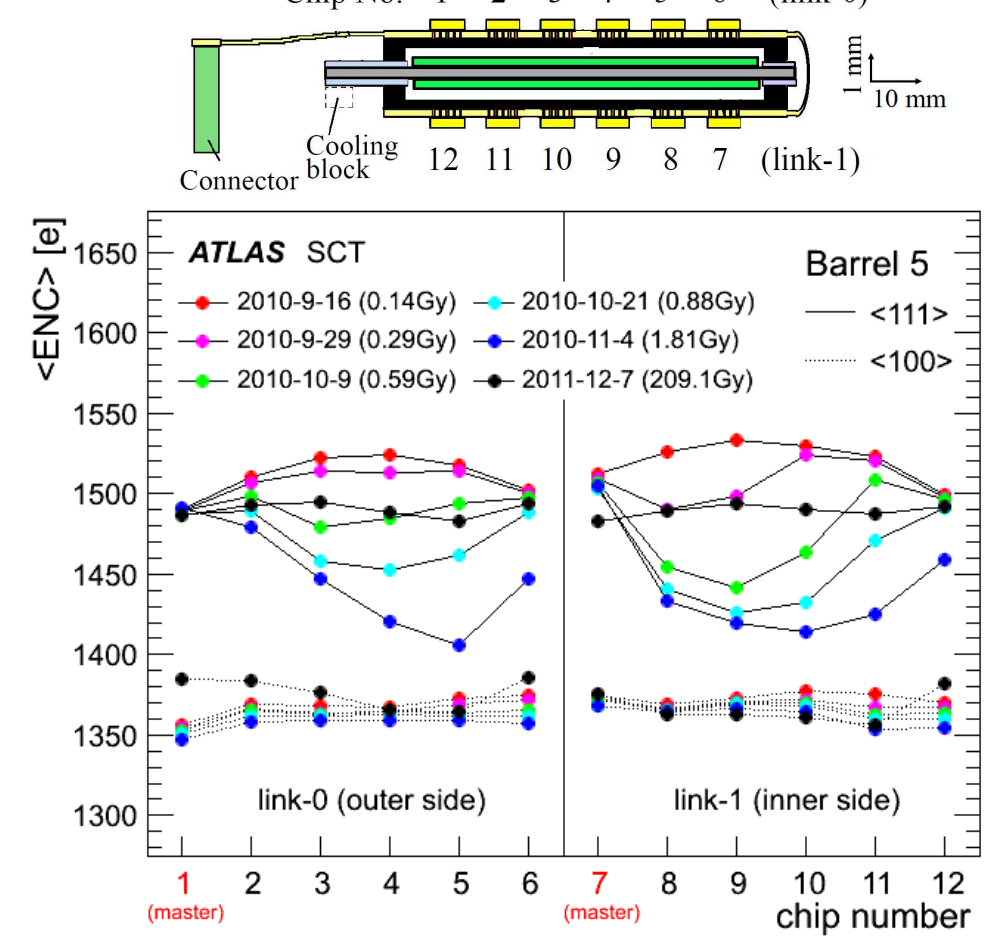

Figure 15. Chip-averaged noise as a function of chip number for several runs during 2010 (coloured points) and at the end of year 2011 (black points) for barrel 5. Chips with $<100>$ sensors are plotted separately. Chip numbers and locations are indicated in the top diagram.

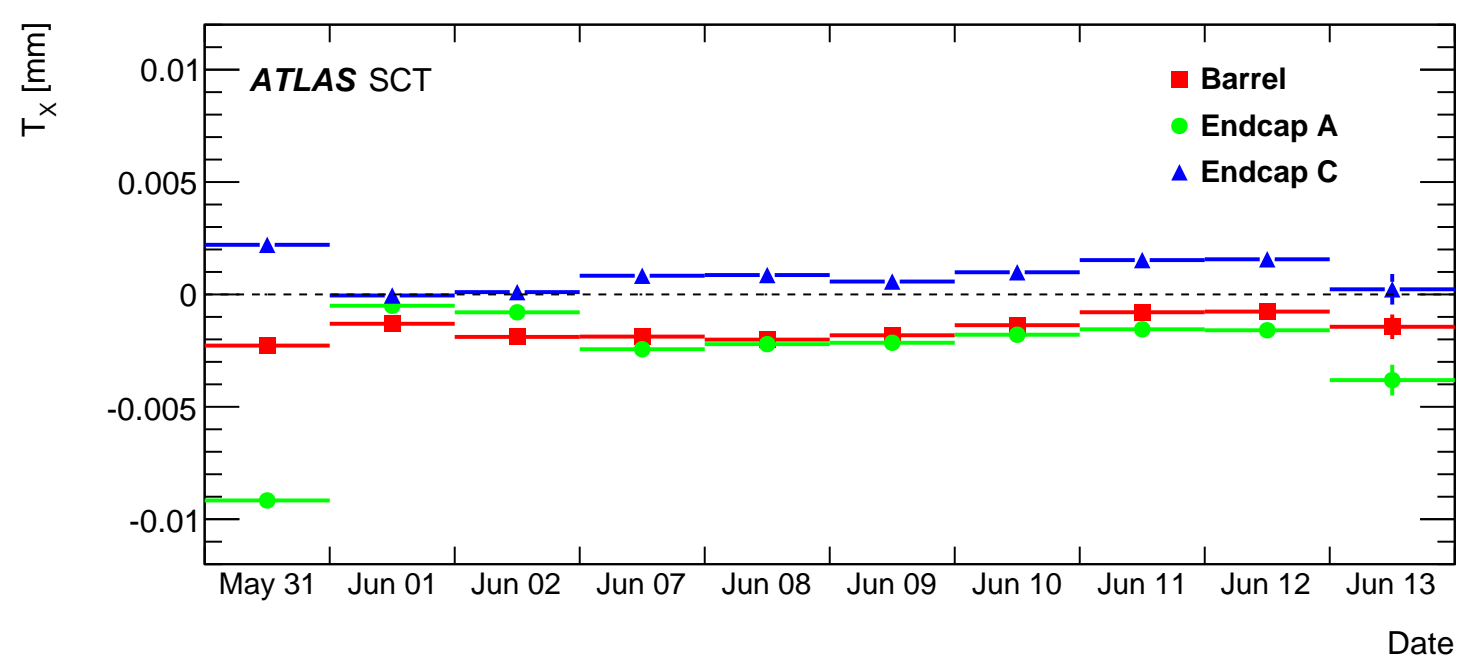

Figure 16. Subsystem-level track-based alignment corrections in the global $x$ coordinate, $T_{x}$, performed on a run-by-run basis starting from a common set of alignments. The subset shown covers a two-week period during May and June 2011. The first data points here show the effect on the alignment constants of the toroid-magnet ramping. 

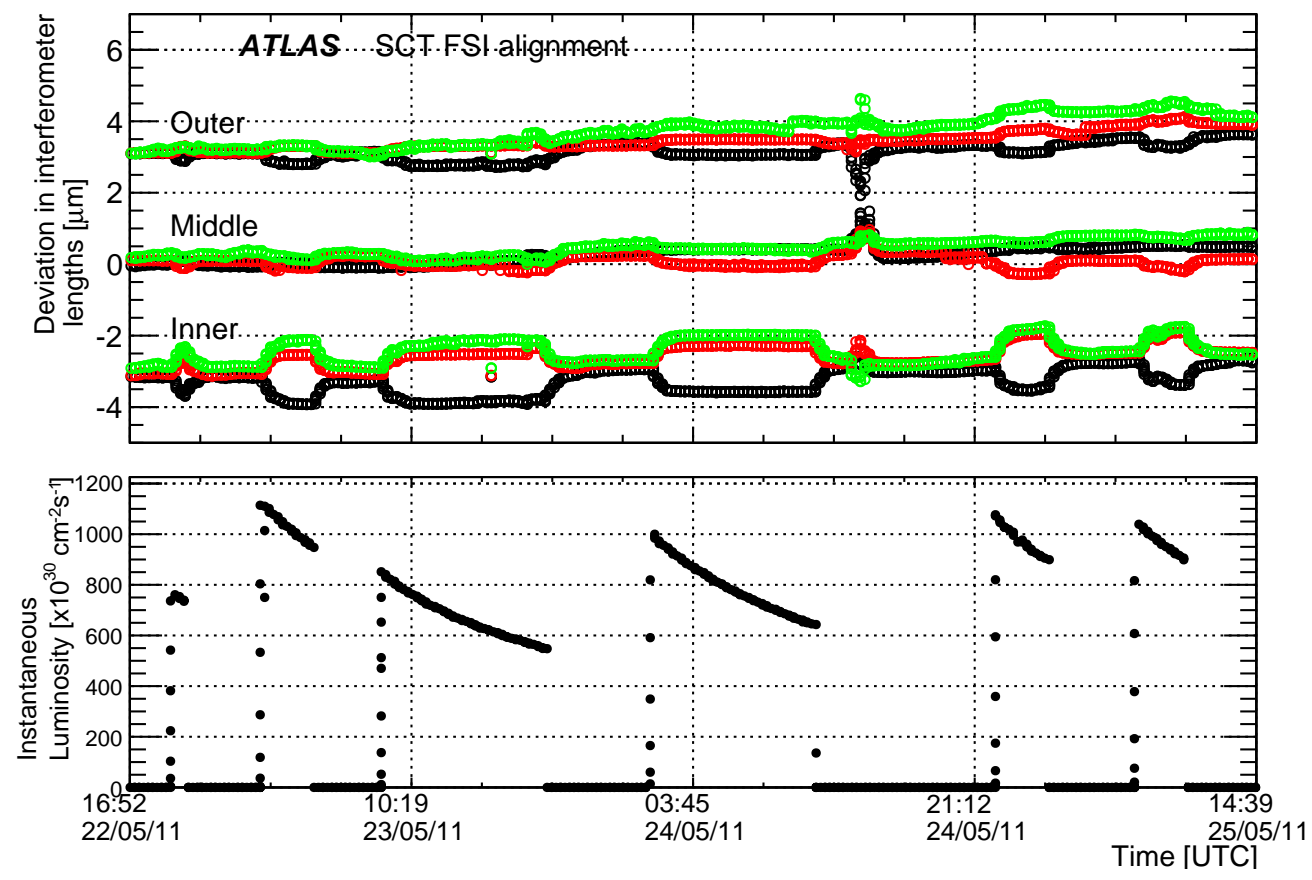

Figure 17. Length deviation of barrel-flange interferometers during three days of LHC collision data-taking in May 2011. In the upper plot, the lower three curves (shifted by $-3 \mu \mathrm{m}$ ) show the measurements from the inner layer, the three curves in the middle show the measurements from middle layer, the upper three curves (shifted by $+3 \mu \mathrm{m}$ ) show the measurements from the outer layer. The colours correspond to those in figure $4 \mathrm{c}$. The lower plot shows the instantaneous LHC luminosity.

Figure 18a shows the effect observed in the FSI during ramping of the solenoid magnetic field in December 2009. The upper plots show the current in the magnet and the temperature of the gas in the SCT; the lower plot shows the displacement of the barrel-flange grid lines. Displacements up to $3 \mu \mathrm{m}$ are observed while the field is ramping but the detector is observed to return to its initial position at the end of the ramping process. In figure 18b, the effect of the toroid field is shown for three barrel-flange lines. A displacement of $3 \mu \mathrm{m}$ is correlated in time with a toroidal current variation of $20 \mathrm{kA}$. This corresponds to the movement observed in the track-based alignment at the end of May 2011 shown in figure 16. Another movement of about $0.5 \mu \mathrm{m}$ can be correlated with the short luminosity pulse observed on the same day at around 20:00 $\mathrm{h}$.

\subsection{Intrinsic hit efficiency}

The intrinsic detector efficiency measures the probability of a hit being registered when a charged particle traverses the sensitive part of an operational detector element. Disabled sensors and chips are excluded from the measurement. Both a high intrinsic efficiency and a low non-operational fraction are essential to ensure good-quality tracking.

The intrinsic efficiencies of the modules are measured by extrapolating well-reconstructed tracks through the detector and counting the numbers of hits (clusters) on the track and 'holes' where a hit would be expected but is not found. The track extrapolation uses the full track fit described in section 4.1 to compute the intersections of the track with all modules along its trajectory. 

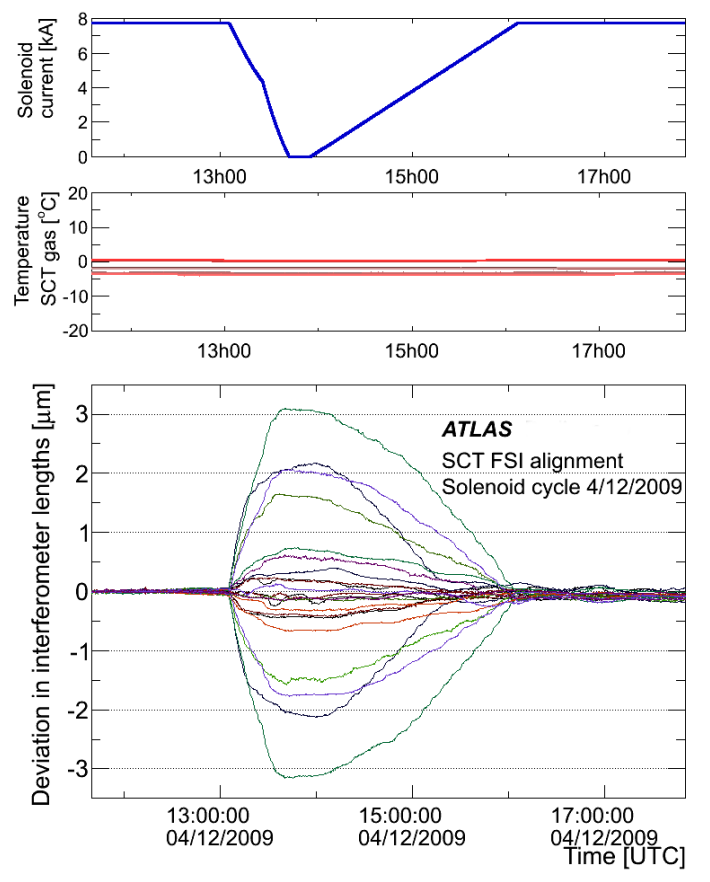

(a)

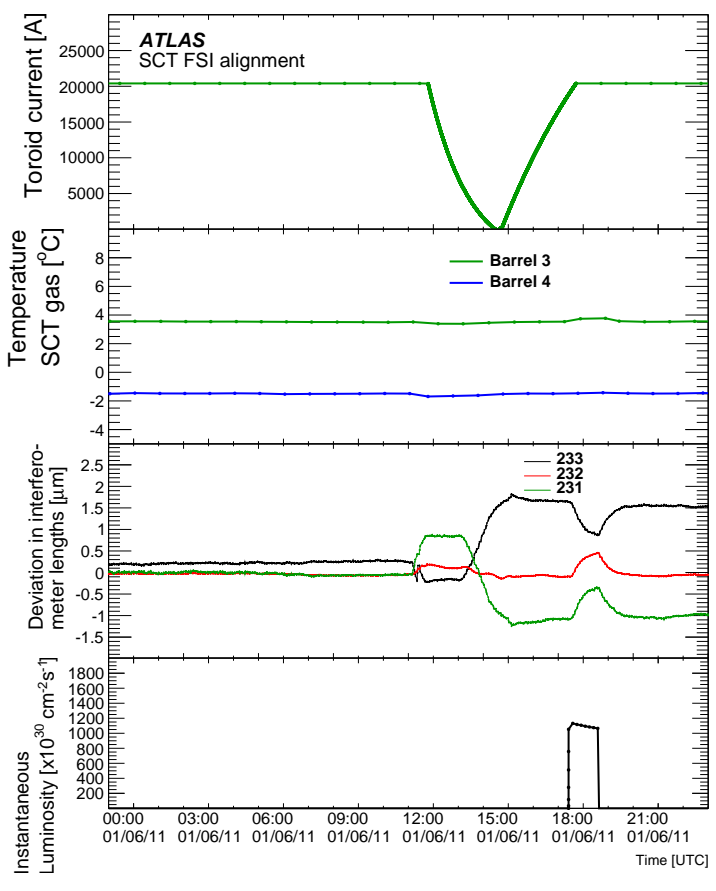

(b)

Figure 18. Length deviations of SCT barrel-flange interferometers (a) during a cycle of the ATLAS solenoid and (b) during a toroid-magnet ramping. In (a) the upper plot shows the solenoid current, the middle plot the temperature of the gas inside the SCT volume and the lower plot the measured deviations. In (b) the plots show, from top to bottom, the toroid current, the gas temperature, the measured deviations and the instantaneous luminosity.

If a module side does not have a cluster associated to the track and the intersection point is more than $3 \sigma$ from the edge of the sensitive area, the absence is called a hole. The efficiency, $\varepsilon$, is defined as the ratio of the number of clusters found to the number expected:

$$
\varepsilon=\frac{N_{\text {clusters }}}{N_{\text {clusters }}+N_{\text {holes }}}
$$

where $N_{\text {clusters }}$ is the number of clusters found and $N_{\text {holes }}$ is the number of holes.

Combined inner detector tracks used to measure the efficiency must have at least six hits in the SCT excluding the hit or hole under consideration, and transverse momentum $p_{\mathrm{T}}>1 \mathrm{GeV}$. Events containing more than 250 reconstructed tracks are excluded from the efficiency measurement, to reduce biases from reconstruction algorithms in high-occupancy events. To reduce any bias due to the track fitting and pattern recognition criteria, which may be affected by residual misalignments, clusters not already assigned to a track but within $200 \mu \mathrm{m}$ of an intersection are included in $N_{\text {clusters }}$ in equation (6.1) and removed from $N_{\text {holes. }}$. From a study of the residual distance of the second-nearest cluster to a track it is estimated that only $4.4 \%$ of such clusters are due to random associations or noise, and most result from hit-to-track assignment criteria applied during track reconstruction. The inclusion of these clusters increases the efficiency by $0.014 \%$. The distance for inclusion of unassigned clusters is varied in the assessment of systematic uncertainties. 


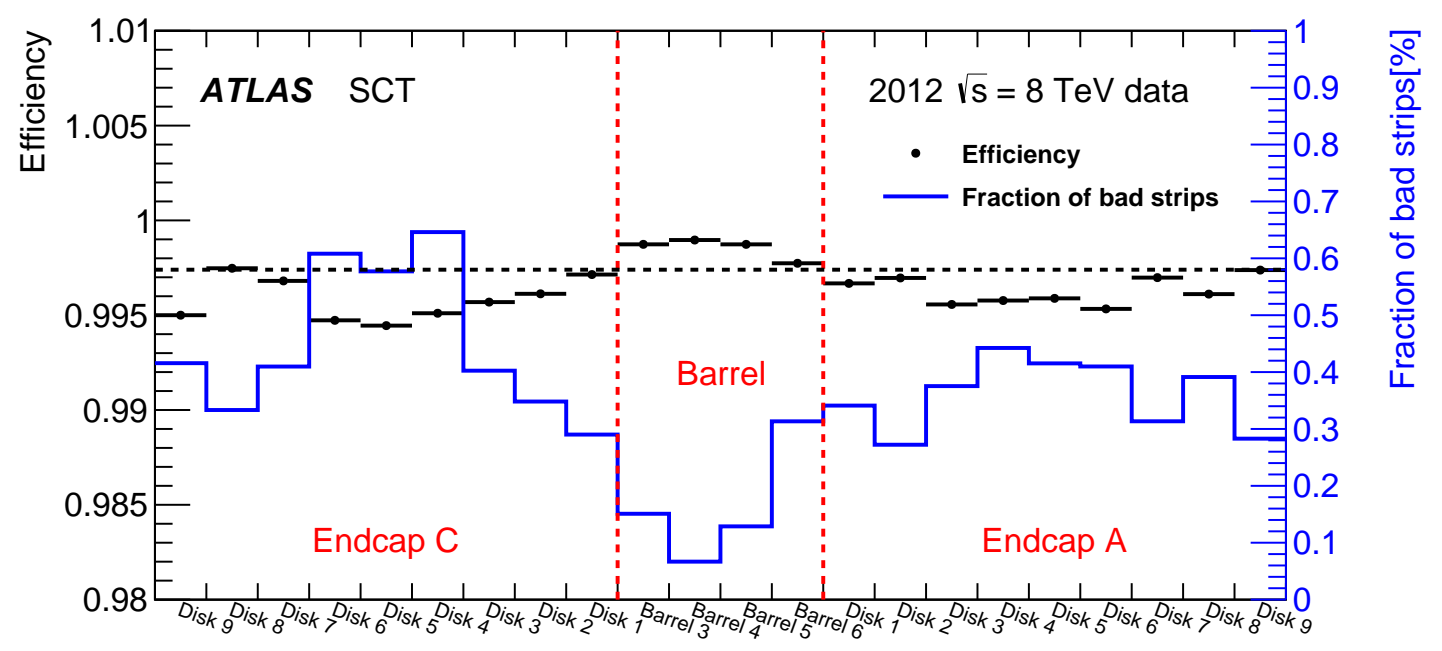

Figure 19. Mean intrinsic hit efficiency for each layer of the SCT measured in proton-proton data at $\sqrt{s}=$ $8 \mathrm{TeV}$ in 2012. The dashed line indicates the overall mean value. The blue line and right-hand axis show the fraction of strips in the layer which are disabled (dead or noisy).

Non-functioning complete module sides and chips (see section 3) are not included in the calculation of the intrinsic efficiency; these amount to $\sim 1 \%$ of the detector. The measured inefficiency contains a contribution from isolated dead strips, including those which are disabled in reconstruction due to noise, for which no correction is applied.

The measured efficiency for each barrel and each endcap disk in 2012 is shown in figure 19. These measurements use data recorded in a proton-proton run with low pile-up, with less than one interaction per bunch-crossing on average. The overall efficiency is $(99.74 \pm 0.04) \%$, where the error is systematic. The statistical error is negligible. The systematic uncertainty was estimated by varying the distance within which unassigned hits are included from zero to $500 \mu \mathrm{m}\left({ }_{-0.014}^{+0.019} \%\right)$ and by considering the change when only module sides for which a hit was registered in the other side are used in the measurement $(+0.036 \%)$. The overall systematic uncertainty is taken as the (symmetrised) sum in quadrature of these two contributions. As a cross-check, the efficiency was determined using different track selection criteria: varying the mimimum transverse momentum requirement between $0.5 \mathrm{GeV}$ and $2 \mathrm{GeV}$, reducing the requirement on the minimum number of other SCT hits from six to five, and placing requirements on the number of hits in the pixel or TRT detectors. These variations give values within the uncertainty estimated by requiring a hit on the other module side. The efficiencies measured in the barrel and endcap regions separately are $(99.86 \pm 0.03) \%$ and $(99.59 \pm 0.05) \%$ respectively. The efficiency measured in $2012 p p$ collisions in the barrel region is very similar to the value of $(99.78 \pm 0.01$ (stat.) \pm 0.01 (syst.))\% measured in cosmic-ray data in 2008 [5], indicating little change in detector performance over this period.

The variation in efficiency from layer to layer seen in figure 19 primarily results from differences in the proportion of isolated disabled strips in each layer. The fraction of such strips in each layer is also shown in figure 19, and a clear anti-correlation with efficiency is seen. While there is some dependence on the distribution of disabled strips (i.e. single, pairs or larger groups), 


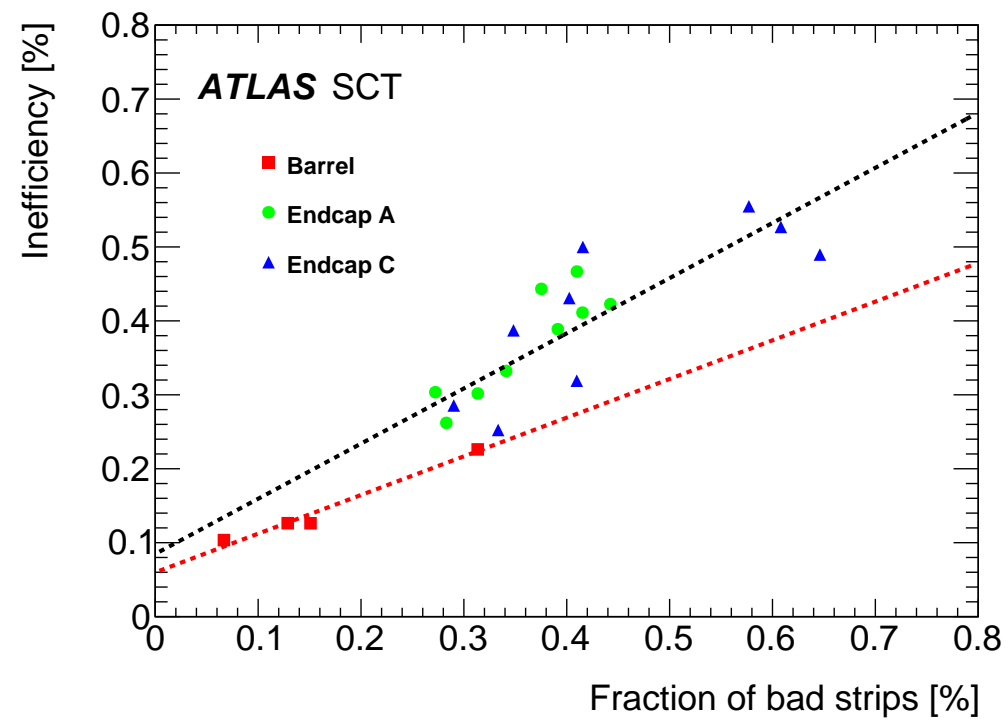

Figure 20. Mean intrinsic hit inefficiency for each layer of the SCT measured in proton-proton collision data at $\sqrt{s}=8 \mathrm{TeV}$ in 2012 as a function of the fraction of individual strips in the layer which are dead or disabled due to noise. The statistical errors on the inefficiency values are smaller than the size of the data points. The dashed lines show the results of linear fits for the barrel and endcaps separately.

on average the inefficiency is linearly dependent on the total fraction. This dependence is shown in figure 20, where it can be seen that the inefficiency is dominated by the contribution from disabled strips. A $1 \%$ fraction of disabled strips gives rise to an efficiency loss of about $0.52 \%$ in the barrel and $0.75 \%$ in the endcap. The lower dependence in the barrel arises from the larger mean cluster width.

\subsection{Lorentz angle}

Charge carriers in the silicon detectors are subject to the electric field, $\mathbf{E}$, generated by the bias voltage and oriented normal to the module plane, and to the magnetic field from the solenoid, $\mathbf{B}$. In the SCT endcap modules these fields are nearly parallel and the charge carriers drift directly towards the electrodes. In the barrel modules these fields are perpendicular and the charge carriers drift along the Lorentz angle, $\theta_{\mathrm{L}}$, with respect to the normal to the sensor plane. The value of Lorentz angle is given by:

$$
\tan \theta_{\mathrm{L}}=\mu_{\mathrm{H}} B=\gamma_{\mathrm{H}} \mu_{\mathrm{d}} B
$$

where $\mu_{\mathrm{H}}$ is the Hall mobility, the product of the charge-carrier mobility in silicon $\mu_{\mathrm{d}}$ and the Hall factor $\gamma_{\mathrm{H}}$, which is of order unity. The charge-carrier mobility depends on the bias voltage, the thickness of the depleted region and the temperature [44]. For fully depleted modules, the average shift in collected charge is approximately $10 \mu \mathrm{m}$, which is not negligible with respect to the detector resolution and alignment precision. Measurements of the Lorentz angle for the SCT sensors have previously been made in test-beams [43] and using cosmic-ray data collected prior to LHC beam operation [5]. The latter measurements were shown to be compatible with the model predictions for the charge-carrier mobility of ref. [44], within the uncertainties on these predictions. 


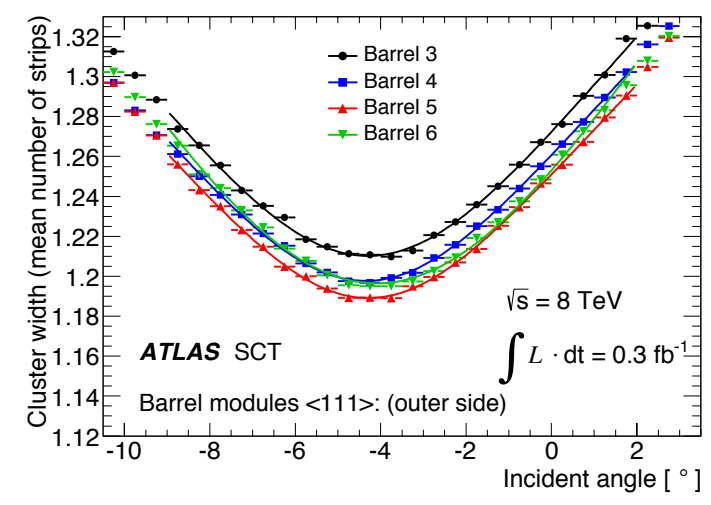

(a)

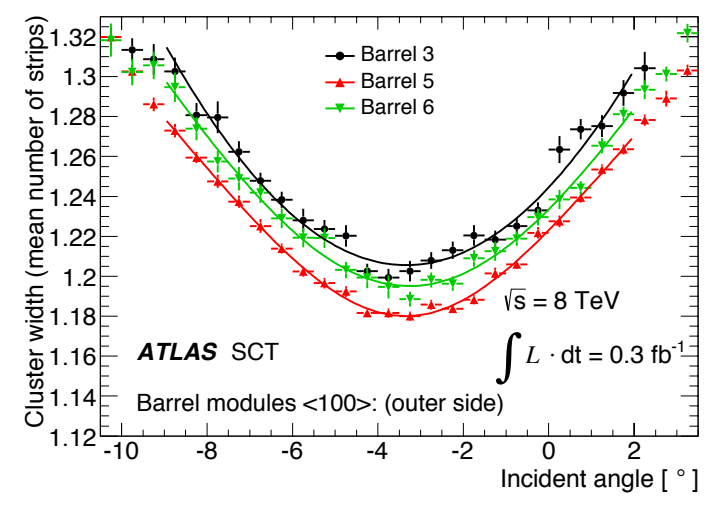

(b)

Figure 21. Cluster-size dependence on the particle incident angle for each SCT barrel for $(a)<111>$ sensors and (b) $<100>$ sensors. The displacement of the minimum from zero is a measurement of the Lorentz angle $\theta_{\mathrm{L}}$.

The very high number of tracks in the collision data now allows the Lorentz angle to be studied in more detail than was possible using cosmic-ray data. In particular, differences in the charge-carrier mobility mean that the expected Lorentz angle is different for sensors with different crystal lattice orientations. In the studies presented here, sensors with $<111>$ and $<100>$ crystal orientations are considered separately.

The Lorentz angle is measured from the dependence of the cluster size on the incident angle of the particle. When the incident angle equals the Lorentz angle, all charge carriers generated by the particle drift along the particle direction and, apart from charge diffusion, are collected at the same point on the sensor surface, giving a minimum cluster size. The tilt of the barrel modules with respect to the radial direction and correlations between particle transverse momentum and the incident angle for particles originating near the beam axis give rise to a range of possible incident angles for positive and negative particles. Reconstructed tracks are required to have $p_{\mathrm{T}}>400 \mathrm{MeV}$, limiting the possible range of incident angles to approximately $-30^{\circ}<\phi_{\text {local }}<-5^{\circ}$ for positive particles and $-15^{\circ}<\phi_{\text {local }}<10^{\circ}$ for negative particles. Thus, only the tracks of negatively charged particles are used to measure the Lorentz angle in collision data.

The dependence of the cluster size on the incident angle $\phi_{\text {local }}$ is shown in figure 21 for data from each barrel. The figures on the left and on the right show the results for $\langle 111\rangle$ and $<100\rangle$ sensors, respectively. Data are fitted using a convolution of the function:

$$
f\left(\phi_{\text {local }}\right)=a\left|\tan \phi_{\text {local }}-\tan \theta_{\mathrm{L}}\right|+b
$$

with a Gaussian distribution. The fitted parameters are the Lorentz angle, $\theta_{\mathrm{L}}$, the shape parameters, $a$ and $b$, and the width of the Gaussian. The electric field in the silicon, and thus the local Lorentz angle, varies with distance from the electrodes. The measured values are averages over particle paths within a sensor. Fits are performed separately for the inner and outer sides of each barrel layer for several datasets recorded during 2011 and 2012. Results from the two sides of a layer are compatible and are combined. The variation in Lorentz angle expected from the spread of 
module temperatures in a barrel of about $1^{\circ} \mathrm{C}$ is negligible. Systematic uncertainties arising from the choice of fit function are assessed by using an alternative asymmetric function, in which the slope parameter $a$ is allowed to take different values above and below the minimum. The mean difference in measured Lorentz angle in each layer is taken as a systematic uncertainty correlated among datasets, while the r.m.s. spread of the differences is used as an estimate of the systematic uncertainty uncorrelated among datasets. In addition, a fit was performed on cosmic-ray data with no magnetic field; the measured value of the Lorentz angle is compatible with zero, and the precision of this check $\left(0.12^{\circ}\right)$ is included as an additional systematic uncertainty, correlated among all measurements.

Figure 22 shows measured values of the Lorentz angle for the innermost barrel for runs during stable-beam periods in 2011 and 2012. The variation in measured Lorentz angle over this period is no more than $0.1^{\circ}$. The other barrel layers show similar stability.

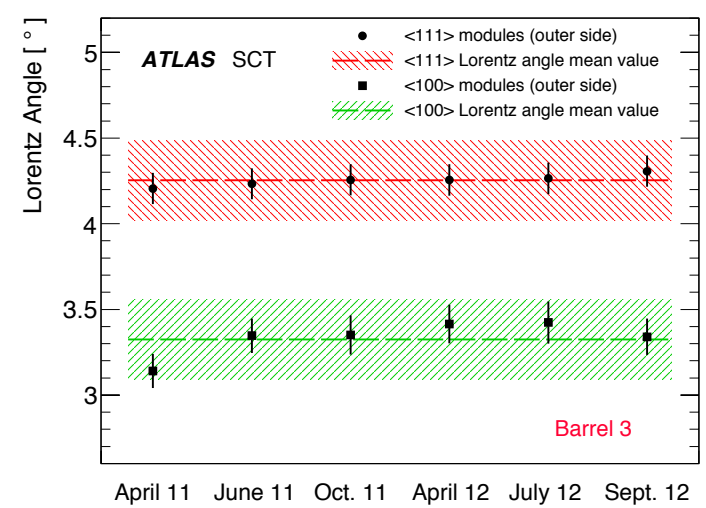

(a)

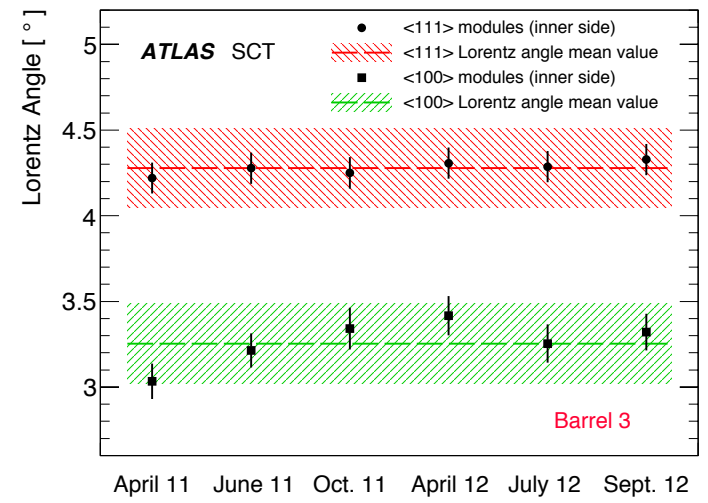

(b)

Figure 22. Average fitted values of the Lorentz angle for $<111>$ and $<100>$ sensors in the innermost barrel for stable-beam running periods in 2011 and 2012; (a) shows results for the outer modules sides, while (b) shows the inner module sides. The error bars show the combined statistical and uncorrelated systematic uncertainties. In each case, the dashed line shows the average over the full 2011 and 2012 datasets, with the correlated systematic uncertainty indicated by the shaded band.

The measured values of the Lorentz angle in the $2 \mathrm{~T}$ magnetic field, averaged over all datasets, are shown in tables 6 and 7 and in figure 23. They are compared with the expectation using the charge-carrier mobility from the models in refs. [44] and [45]. The main uncertainty in the model predictions arises from knowledge of the drift velocity; the uncertainty in this is assumed to be $5 \%$, based on measurements using the time-of-flight technique. Non-uniformity of the electric field and uncertainties in temperature and magnetic field also contribute. The measurements are compatible with the model predictions within at most twice the estimated uncertainties on these predictions. The measured values of the Lorentz angle for the $<100>$ sensors are approximately $1^{\circ}$ lower than those obtained for $<111>$ sensors. This is contrary to the expectation that a higher expected charge-carrier mobility in the $<100>$ sensors should result in a higher value of the Lorentz angle [45]. 
Table 6. Measured values of the Lorentz angle in $<111>$ modules, in a $2 \mathrm{~T}$ magnetic field at the average operational temperature in the period from 2011 to 2012, compared with the model expectations of Jacoboni et al. [44] and Becker et al. [45]. The uncertainties on the measurements include both statistical and systematic contributions; those on the model predictions arise from uncertainties in the charge-carrier mobility.

\begin{tabular}{|lcccc|}
\hline \multicolumn{4}{|c|}{$<111>$} \\
\hline Layer & $T\left[{ }^{\circ} \mathrm{C}\right]$ & Measured $\left.\theta_{\mathrm{L}}{ }^{\circ}\right]$ & Jacoboni et al. Model $\left.\theta_{\mathrm{L}}{ }^{\circ}\right]$ & Becker et al. Model $\left.\theta_{\mathrm{L}}{ }^{\circ}\right]$ \\
\hline Barrel 3 & -2.1 & $4.28 \pm 0.23$ & $3.88 \pm 0.29$ & $3.50 \pm 0.34$ \\
Barrel 4 & -1.7 & $4.26 \pm 0.22$ & $3.87 \pm 0.29$ & $3.50 \pm 0.33$ \\
Barrel 5 & -1.3 & $4.30 \pm 0.15$ & $3.86 \pm 0.29$ & $3.49 \pm 0.33$ \\
Barrel 6 & 5.6 & $4.07 \pm 0.15$ & $3.69 \pm 0.26$ & $3.35 \pm 0.30$ \\
\hline
\end{tabular}

Table 7. Measured values of the Lorentz angle in $<100>$ modules, in a $2 \mathrm{~T}$ magnetic field at the average operational temperature in the period from 2011 to 2012, compared with the model expectations of Becker et al. [45]. The uncertainties on the measurements include both statistical and systematic contributions; those on the model predictions arise from uncertainties in the charge-carrier mobility. Barrel 4 has no $<100>$ modules.

\begin{tabular}{|lccc|}
\hline \multicolumn{3}{|c|}{$<100>$} \\
\hline Layer & $T\left[{ }^{\circ} \mathrm{C}\right]$ & Measured $\left.\theta_{\mathrm{L}}{ }^{\circ}\right]$ & Becker et al. Model $\left.\theta_{\mathrm{L}}{ }^{\circ}\right]$ \\
\hline Barrel 3 & -2.1 & $3.25 \pm 0.23$ & $4.01 \pm 0.33$ \\
Barrel 5 & -1.3 & $3.28 \pm 0.15$ & $4.00 \pm 0.32$ \\
Barrel 6 & 5.6 & $3.24 \pm 0.15$ & $3.82 \pm 0.28$ \\
\hline
\end{tabular}

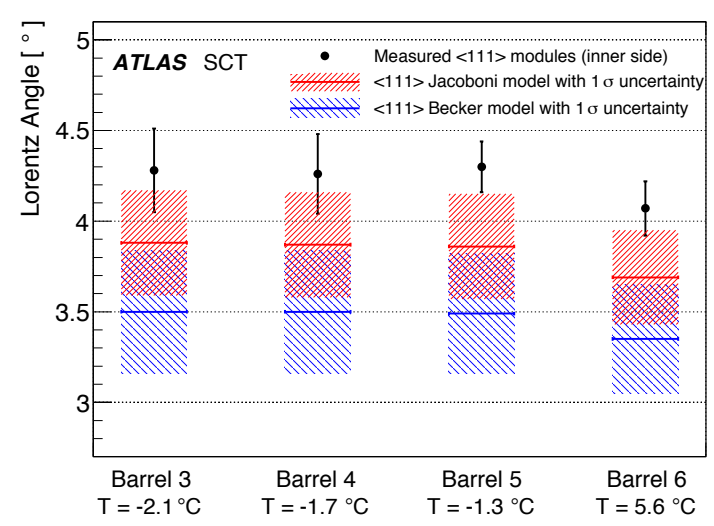

(a)

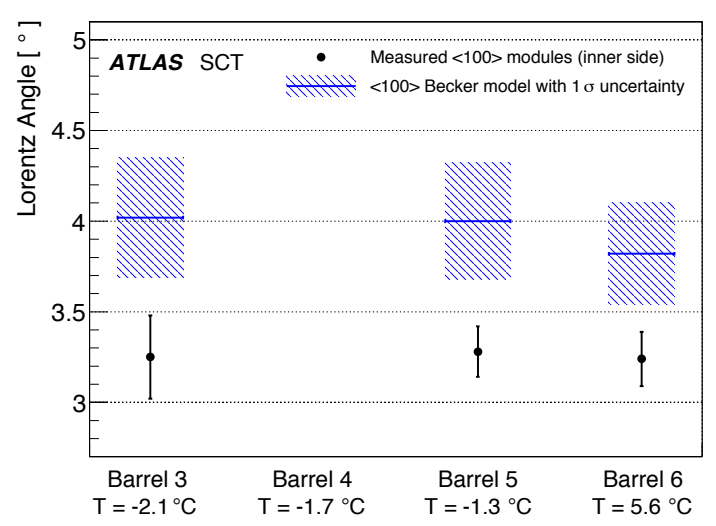

(b)

Figure 23. Measured values of the Lorentz angle $\theta_{\mathrm{L}}$ in each layer of the SCT barrel (black points) for (a) $<111>$ sensors and (b) $<100>$ sensors. The error bars include the statistical and systematic uncertainties. The red and blue lines show the expectations of the models of refs. [44] and [45] respectively, with the $1 \sigma$ uncertainties indicated by the hashed areas. The mean temperature of each layer is shown with the barrel number; a higher temperature is maintained in the outermost layer, barrel 6 , resulting in a lower expected Lorentz angle. 


\subsection{Energy loss and particle identification}

Although the SCT is not designed to perform measurements of energy loss, $\mathrm{d} E / \mathrm{d} x$, and particle identification, some discriminating power is available from the number of time bins above threshold and the number of strips in a cluster. A more heavily ionising particle which deposits more charge will produce a larger pulse height, which is more likely to be above threshold in the time bin corresponding to the bunch crossing after the trigger. Moreover, a shorter path length is required to deposit enough charge to be above threshold, so such a particle will tend to produce wider clusters. Both of these expectations are exploited by calculating a quantity related to the energy loss of a particle, $\mathrm{d} E / \mathrm{d} x_{\mathrm{SCT}}$, given by:

$$
\mathrm{d} E / \mathrm{d} x_{\mathrm{SCT}}=\frac{1}{N} \sum_{i} w_{i} \cos \alpha_{i}
$$

where the sum runs over all the strips in all clusters assigned to the track, $N$ is the number of clusters assigned to the track and $w_{i}$ is a weight depending on the number of time bins which are above threshold for that strip; by default $w_{i}$ is set equal to the number of time bins above threshold, except for illegal time-bin patterns, which are given a weight of zero. The factor of $\cos \alpha_{i}$, where $\alpha_{i}$ is the angle between the track and the normal to the silicon sensor, corrects for the path length of the particle within the silicon.

Figure 24a shows $\mathrm{d} E / \mathrm{d} x_{\mathrm{SCT}}$ as a function of momentum multiplied by charge for barrel-region tracks from minimum bias data collected in 2010. To increase the number of protons in the sample, the fraction of particles originating from secondary interactions in detector material is enhanced by selecting tracks with large impact parameters with respect to the primary interaction vertex. The protons can clearly be seen in the figure, forming a distinct band of highly ionising particles in the low-momentum positive-charge region. This band is not seen for negative particles: the number of anti-protons in the sample enhanced in the products of secondary interactions is expected to be much smaller.

Particle identification is performed using a likelihood method. The probability density functions for different particles are determined, in ranges of momentum, by fitting Gaussians to distributions of $\mathrm{d} E / \mathrm{d} x_{\mathrm{SCT}}$ for particles identified as protons, kaons or pions on the basis of $\mathrm{d} E / \mathrm{d} x$ measured in the pixel detector [46]. Figure 24b shows the efficiency for tagging protons, defined as the fraction of particles identified as protons using the pixel detector which are tagged as protons using the SCT, as a function of the mistag rate for kaons; the mistag rate for kaons is defined as the fraction of particles identified as kaons by the pixel detector which are tagged as protons by the SCT. In the momentum range $400-550 \mathrm{MeV}$, a tagging efficiency for protons of more than $90 \%$ can be achieved, with a mistag rate of less than $30 \%$ for kaons. The corresponding mistag rate for pions is less than $4 \%$.

Although the SCT has been shown to have some discriminating power, its use for particle identification is limited to momenta below about $600 \mathrm{MeV}$. However, the peak position of the $\mathrm{d} E / \mathrm{d} x_{\mathrm{SCT}}$ distribution for protons may become a useful tool for monitoring radiation damage to the sensors in future. The position of this peak was stable at the 5-10\% level during 2010-2012 when radiation damage was negligible. Variations arising from drifts in detector timing relative to the ATLAS trigger time were observed. Future use of this peak for monitoring depends on the 


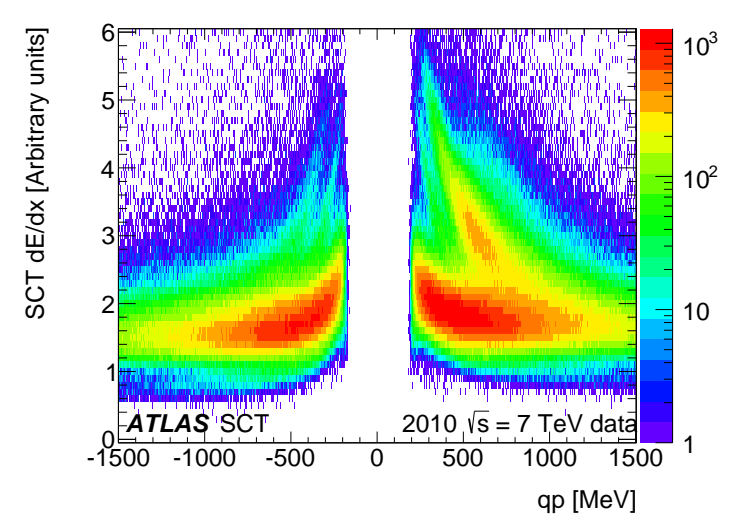

(a)

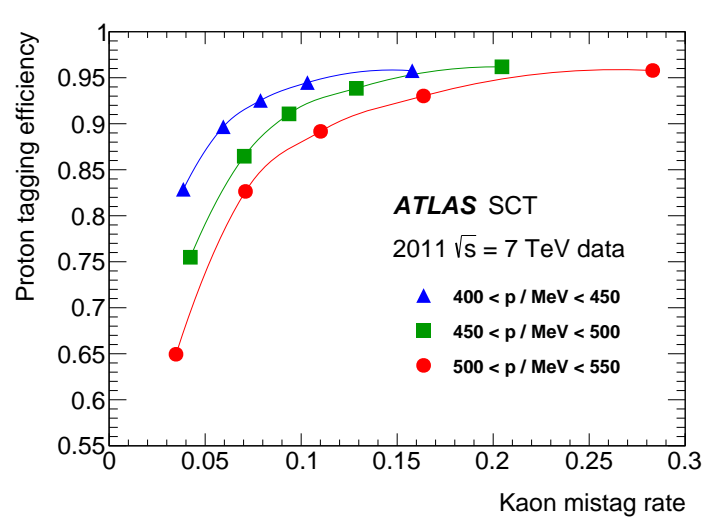

(b)

Figure 24. (a) Energy loss measured in the SCT (see text for definition) as a function of momentum multiplied by charge for a sample of tracks enhanced in products of secondary interactions (see text). Tracks are taken from minimum bias data collected during 2010, and required to be in the barrel region of the detector. (b) Proton tagging efficiency as a function of the kaon mistag rate measured in 2011 minimum bias data.

stability of the detector timing and threshold, and will require data recorded in level mode (see section 2.2) with time-bin information.

\subsection{Measurement of $\delta$-ray production}

Energy deposition by charged particles in silicon leads to the production of secondary electrons, called $\delta$-rays, that can travel distances of several hundred microns and produce secondary ionisation. These $\delta$-rays may give hits in neighbouring strips that were not traversed by the primary particle, and thus broaden clusters and bias the position measurement. It is thus important to check that the rate and spectrum of $\delta$-rays are simulated correctly.

The production of $\delta$-rays in the barrel sensors has been measured and compared to simulation by counting the number of clusters that are broader than expected from the angle of the track traversing the sensor. The measurement uses good-quality tracks with at least eight SCT hits from about 257 million minimum-bias events, together with about 300 million simulated events. Tracks sharing one or more clusters with another track are removed from the analysis; the remaining contamination of clusters arising from charge deposited by two or more primary particles is negligible.

The idea is illustrated in figure 25. Ignoring the effects of charge diffusion, the expected width, $w_{\mathrm{e}}$, of a cluster from a track with incident angle $\phi_{\text {local }}$ is given by

$$
w_{\mathrm{e}}=t\left(\tan \phi_{\text {local }}-\tan \theta_{\mathrm{L}}\right)
$$

where $t$ is the sensor thickness and $\theta_{\mathrm{L}}$ is the Lorentz angle. The emission of a $\delta$-ray may increase the observed width, $w_{\mathrm{o}}$, as illustrated in figure 25. To identify $\delta$-rays, clusters for which $w_{\mathrm{e}}<$ $80 \mu \mathrm{m}$ are selected. The primary charge from such a particle can extend over at most two strips, and observed clusters larger than this arise primarily from $\delta$-ray production. The production of a single $\delta$-ray adds strips to one side of the cluster, leading to a shift in the cluster centroid, and 
a shift in the track residual ${ }^{8}$ of approximately $\left(w_{\mathrm{o}}-w_{\mathrm{e}}\right) / 2$. An example residual distribution for clusters of width six strips is shown in figure 26a. The peak at $\sim 0.2 \mathrm{~mm}$ corresponding to single $\delta$-ray production is superimposed on a background centred around zero which arises from effects such as multiple $\delta$-ray production. The number of single $\delta$-rays is estimated by fitting such residual distributions to the sum of two Gaussian functions: one for the signal peak with a mean away from zero and one for the background with a mean near zero. The choice of function is empirical, and is found to model the distributions well, giving $\chi^{2} /$ d.o.f values between 1.5 and 1.8 .

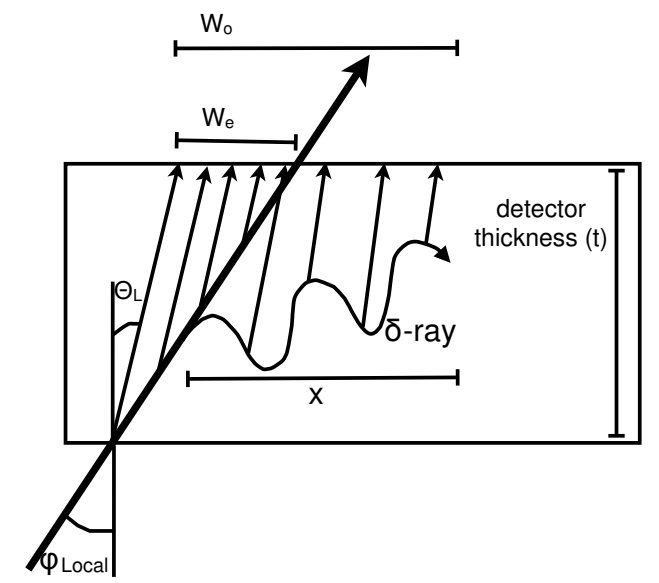

Expectation from track with $\delta$-ray

Figure 25. Sketch of the geometric meaning of expected cluster width $w_{\mathrm{e}}$ and observed width $w_{\mathrm{o}}$. Only the two-dimensional projection of the three-dimensional path length $L$ is shown. Here, $x$ is the distance traversed by a particular $\delta$-ray as described in equation (6.6).

In order to quantify $\delta$-ray production and study the dependence on various parameters, the differential probability for a particle to emit a $\delta$-ray that travels a distance $x$ in the $r-\phi$ plane (i.e. perpendicular to the strip direction) is modelled as:

$$
\mathrm{d} P_{\delta}=\frac{A L}{\rho} e^{-x / \rho} \mathrm{d} x
$$

where $\rho$ is the range (in the $r-\phi$ plane) of the $\delta$-rays, to be determined. The integrated probability from $x=0$ to $x=\infty$ is the normalisation $A L$, where the dependence on path length in silicon, $L$, is explicitly shown since $\delta$-ray production must scale with $L$. The range $\rho$ is obtained by performing Gaussian fits to the residual distributions to determine the number of single $\delta$-rays in bins of observed width, and fitting the resulting numbers to an exponential distribution. An example, for tracks with momentum $p>1.5 \mathrm{GeV}$ and path length in silicon $320<L<380 \mu \mathrm{m}$, is shown in figure $26 \mathrm{~b}$.

The rate of $\delta$-ray production, $A$, is obtained by integrating equation (6.6) to obtain the expected number of single $\delta$-rays in three consecutive $w_{\mathrm{o}}$ bins, $w_{\mathrm{o}}=4,5$ and 6 strips. Clusters with $w_{0}=3$ strips are not used because the signal peak is not well resolved leading to large uncertainties, while for larger values of $w_{\mathrm{o}}$ the statistics are limited.

\footnotetext{
${ }^{8}$ The cluster under consideration is removed from the track fit to remove any bias in the residual.
} 


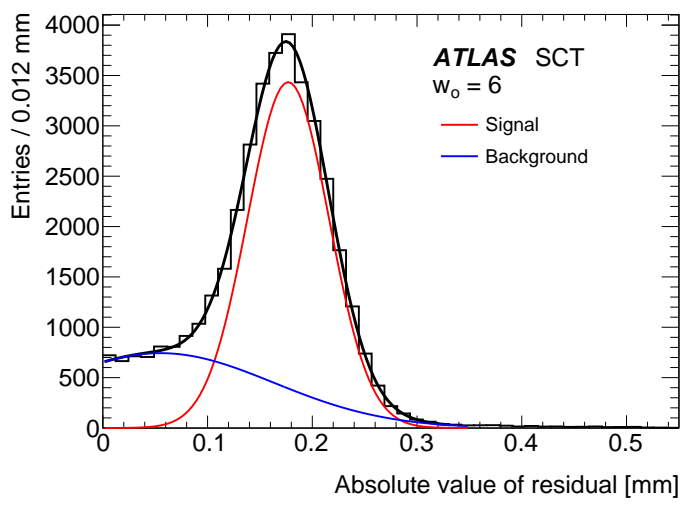

(a)

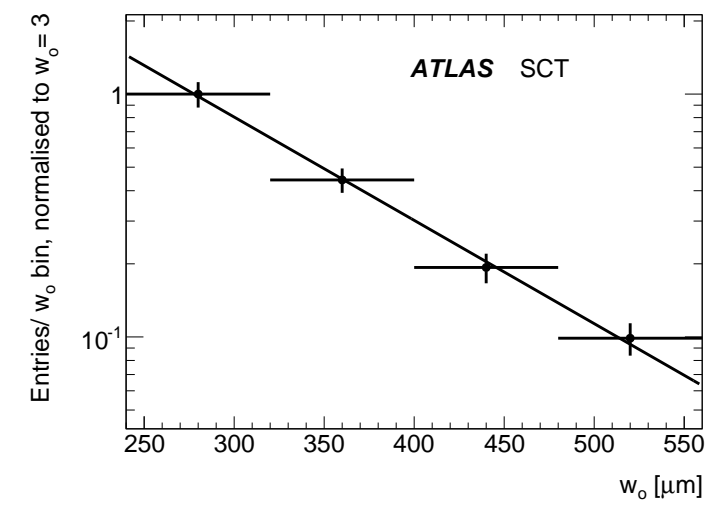

(b)

Figure 26. (a) Distribution of residual magnitude in data for clusters with expected width $w_{\mathrm{e}}<80 \mu \mathrm{m}$, observed width $w_{\mathrm{o}}=6$ strips, track path length in silicon between $275 \mu \mathrm{m}$ and $450 \mu \mathrm{m}$ and track momentum $p>1 \mathrm{GeV}$. The curves show the fit to the sum of two Gaussian distributions. (b) Distribution, normalised to the first bin, of the $\delta$-ray signal (from fits to residual peaks) in bins of $w_{\mathrm{o}}$ for tracks with $p>1.5 \mathrm{GeV}$ and path length in silicon between $320 \mu \mathrm{m}$ and $380 \mu \mathrm{m}$. The fit to an exponential function is superimposed.

The range and rate parameters, $\rho$ and $A$, are determined separately for each barrel, in bins of $L$ and track momentum. Values of both range and rate measured in the separate barrels and in different ranges of $L$ are consistent, and are combined.

The principal systematic uncertainty on these measurements arises from modelling of the background with a single Gaussian distribution, estimated to be $5 \%$ on both range and rate. An additional $3.5 \%$ arises from comparison of the measured values of $\rho$ for different barrels, $L$ and momentum ranges. The estimation of $A$ from the integral of equation (6.6) makes use of the fraction of particles crossing one rather than two strips, which is not directly observed, but is approximated as the ratio of one-strip to two-strip clusters. This approximation, which is valid if the $\delta$-ray production rate is low, adds an uncertainty of $2 \%$ to the rate. The overall systematic uncertainties on the range and rate measurements are $6.1 \%$ and $6.4 \%$ respectively.

Figure 27 shows the measured values of the rate of $\delta$-ray production and their range as a function of track momentum. Data and simulation are seen to be in good agreement, validating the description of $\delta$-ray production in the simulation. The rate is expected to decrease with increasing momentum by about $12 \%$ over the range studied. While the measurements are consistent with this expectation, the errors are too large to resolve this variation. By integrating equation (6.6), the overall probability of a particle emitting a $\delta$-ray with a range of more than $40 \mu \mathrm{m}$ (half the strip pitch) when traversing a typical silicon thickness of $300 \mu \mathrm{m}$ is found to be $1 \%$.

\section{Radiation effects}

The radiation environment in the ATLAS inner detector is complex and comprises a full spectrum of particles (pions, protons, neutrons, photons, etc.), with energies ranging from $\mathrm{TeV}$ down to thermal in the case of neutrons. Close to the interaction point the environment is dominated by particles coming directly from the proton-proton collisions, but at larger radii albedo particles from 


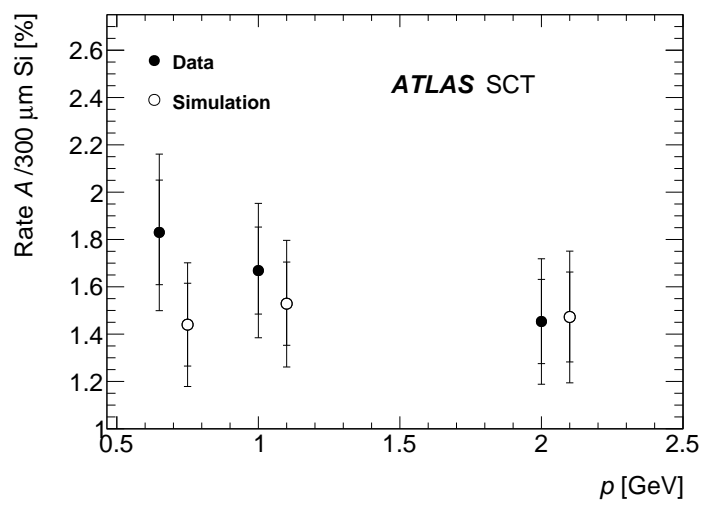

(a)

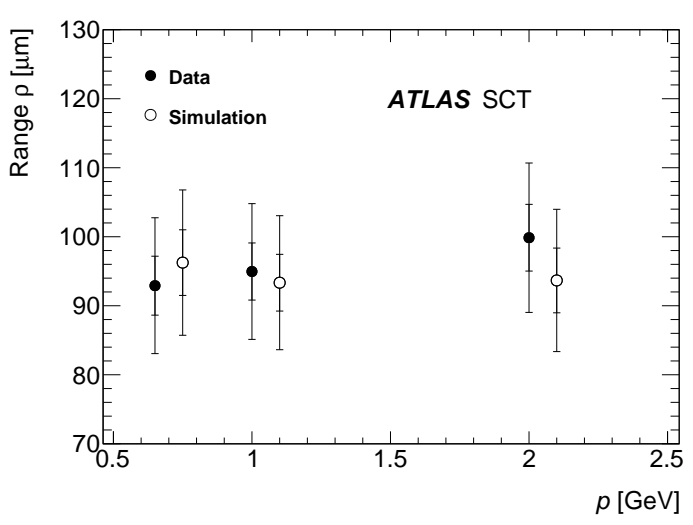

(b)

Figure 27. Measured values of (a) rate per $300 \mu \mathrm{m}$ path length and (b) range for $\delta$-ray production in silicon as a function of track momentum, $p$. The simulation points are artificially shifted by $0.1 \mathrm{GeV}$ for clarity.

high-energy hadron and electromagnetic cascades in the calorimeters dominate the radiation backgrounds in the inner detector. Advanced Monte Carlo event generator and particle transport codes are required to simulate these environments. A review of the radiation backgrounds expected in and around the ATLAS detector can be found in ref. [1]. Results from the most recent simulations for the inner detector are given in section 7.1.

The deleterious effects of radiation on the SCT include: increasing leakage currents; charge accumulation in silicon oxide layers; single-event upsets; decreasing signal-to-noise; changing depletion voltages; and radiation-induced activation of components. Typically both sensors and readout electronics are affected, but in the case of single-event upsets only the readout system is impacted. The SCT system was designed to operate for fluences and doses corresponding to an integrated luminosity of $700 \mathrm{fb}^{-1}$ at a centre-of-mass energy of $14 \mathrm{TeV}$. Up to December 2012 an integrated luminosity of $\sim 29 \mathrm{fb}^{-1}$ had been delivered to the ATLAS experiment, so the effects of radiation on the detectors are expected to be small. However, the large number of SCT modules has allowed robust statistical analyses to be performed. This is particularly true for the detector leakage-current measurements, described in section 7.2, which in turn were used to verify the Monte Carlo fluence predictions. Some degradation effects, such as the change in detector depletion voltage due to radiation-induced impurity states in the silicon, are being observed but are small and still to be studied in detail. Fluence and dose measurements in the inner detector volume were also obtained using a dedicated online radiation monitoring system, described in section 7.3. The particular importance of the radiation monitoring system is to provide ionising-dose information, which is not available from SCT measurements and is important for predicting degradation of the read-out chips.

Single-event upsets have impacted SCT operations, and studies and measurements to understand these effects are described in section 7.4. Some radiation effects were unexpected, such as the anomalous leakage-current increase observed in some CiS sensors leading to additional noise and DAQ issues. The impact of radiation effects on SCT operation and their mitigation is discussed in section 7.5. 


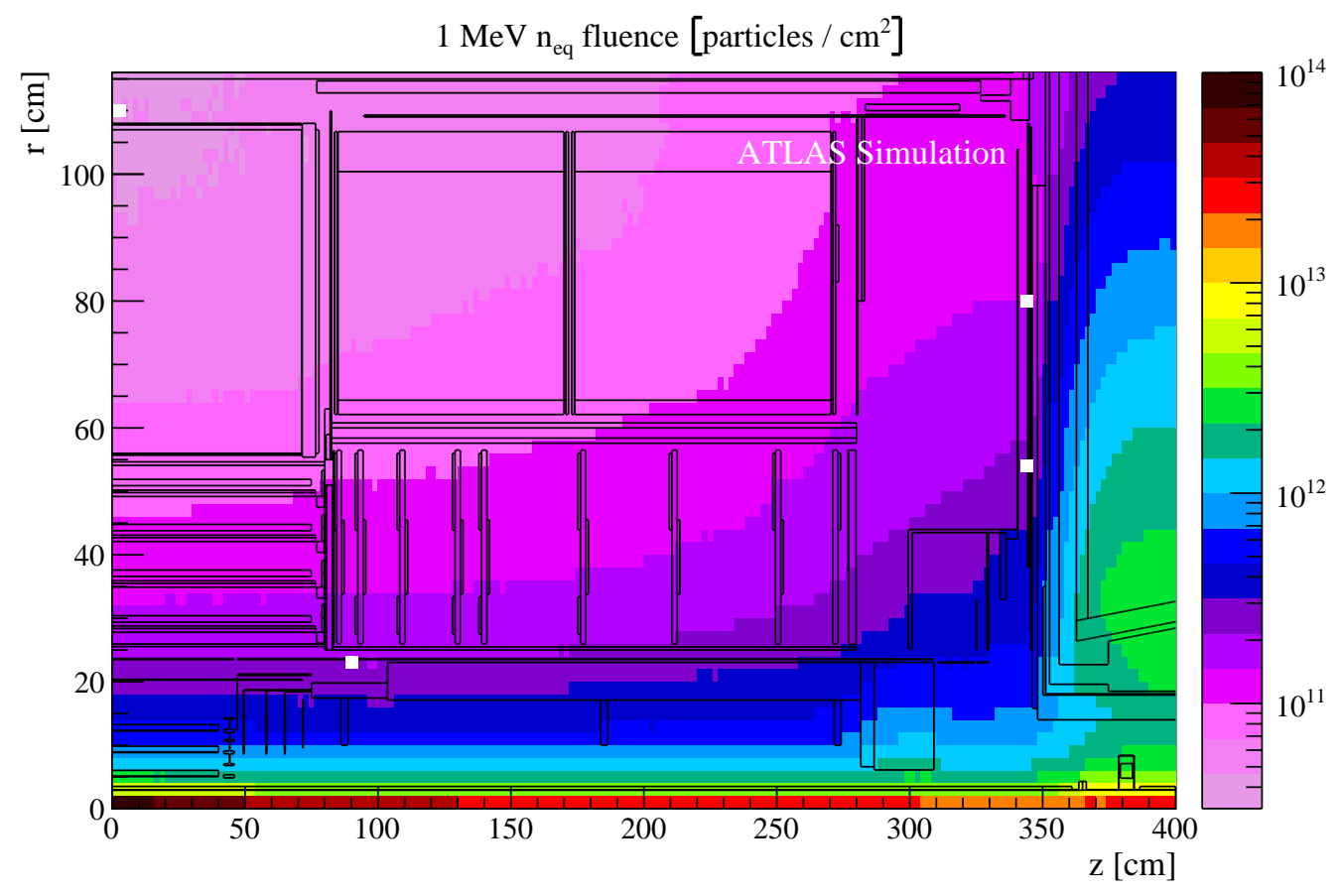

Figure 28. Quarter slice through the inner detector region of the geometry constructed in the FLUKA simulations. The colour contours represent the $1 \mathrm{MeV}$ neutron-equivalent fluences, corresponding to an integrated luminosity of $1 \mathrm{fb}^{-1}$. The four white squares show the positions of the radiation monitors.

\subsection{Simulations and predictions}

Radiation background predictions in the inner detector have been performed with the FLUKA particle transport code $[47,48]$. A detailed description of the geometry and material of the ATLAS detector, shielding and beam-line is constructed within the FLUKA framework. Simulations are performed using the same criteria as described in refs. [1, 49], which also give definitions and explanations of the radiation quantities used. The PYTHIA8 event generator [27] was used to simulate the inelastic proton-proton collisions, for both $\sqrt{s}=7 \mathrm{TeV}$ and $8 \mathrm{TeV}$ corresponding to 2011 and 2012 running respectively. In past studies, the PHOJET [50] event generator was used, but PYTHIA8 is now the preferred choice to take advantage of the significant effort by its authors and the experiments to model and tune PYTHIA8 to describe the $\sqrt{s}=7-8 \mathrm{TeV}$ LHC collision data. The predictions for the fluences in the inner detector are typically $\sim 5 \%$ higher with PYTHIA8 than with PHOJET. A proton-proton inelastic cross section of $71.4 \mathrm{mb}$ at $\sqrt{s}=7 \mathrm{TeV}$ is predicted by PYTHIA8, consistent with measurements [51-54].

The $1 \mathrm{MeV}$ neutron-equivalent fluences [49] for $\sqrt{s}=7 \mathrm{TeV}$, normalised to one $\mathrm{fb}^{-1}$, are shown in figure 28. The rise in the contours towards the endcaps is due to the increasing fluence of albedo neutrons from secondary interactions in the endcap calorimeter material. Average values of the fluences by region are given in table 8. The barrel values are averaged over the full length in $z$ of the layers, while the endcap values are averaged over each ring of a disk (see figure 2). Statistical uncertainties on the predicted fluences are typically a few percent. Comparisons were also made with simulations from the ATLAS GEANT4 framework. Although higher fluence values were 
Table 8. FLUKA predictions $(\sqrt{s}=7 \mathrm{TeV})$ for $1 \mathrm{MeV}$ neutron-equivalent fluences in the SCT. The radiation backgrounds originating from proton-proton collisions are symmetric in $\pm z$ in the inner detector. Statistical uncertainties are typically 3-5\%. Fluences and doses at $\sqrt{s}=8 \mathrm{TeV}$ are predicted to be $\sim 5 \%$ higher than for $\sqrt{s}=7 \mathrm{TeV}[55]$.

\begin{tabular}{|cccccc|}
\hline \multicolumn{5}{|c|}{$1 \mathrm{MeV}$ fluences per $\mathrm{fb}^{-1}\left(\times 10^{11}\right)\left[\mathrm{cm}^{-2}\right]$} \\
Barrel & & Disk & Outer & Middle & Inner \\
\hline 3 & 1.66 & 1 & 1.04 & 1.29 & - \\
4 & 1.30 & 2 & 1.03 & 1.26 & 1.66 \\
5 & 1.07 & 3 & 1.02 & 1.27 & 1.66 \\
6 & 0.92 & 4 & 1.05 & 1.30 & 1.68 \\
& & 5 & 1.08 & 1.32 & 1.73 \\
& & 6 & 1.15 & 1.41 & 1.81 \\
& & 7 & 1.26 & 1.54 & - \\
& & 8 & 1.45 & 1.79 & - \\
& & 9 & 1.66 & - & - \\
\hline
\end{tabular}

Table 9. FLUKA predictions $(\sqrt{s}=7 \mathrm{TeV})$ for $1 \mathrm{MeV}$ neutron-equivalent fluences, ionising dose and thermal neutron fluence at the four different radiation-monitor locations; the positions of these are given in the second and third columns. Fluences and doses at $\sqrt{s}=8 \mathrm{TeV}$ are predicted to be $\sim 5 \%$ higher than for $\sqrt{s}=7 \mathrm{TeV}$.

\begin{tabular}{|lccccc|}
\hline & & \multicolumn{4}{c|}{ Fluence and ionising-dose predictions per $\mathrm{fb}^{-1}$} \\
Location & $r[\mathrm{~cm}]$ & $z[\mathrm{~cm}]$ & $\phi_{1 \mathrm{MeV}}\left[\mathrm{cm}^{-2}\right]$ & Dose $[\mathrm{Gy}]$ & $n_{\text {thermal }}\left[\mathrm{cm}^{-2}\right]$ \\
\hline Pixel support tube & 23 & 90 & $2.3 \times 10^{11}$ & 109 & $0.63 \times 10^{11}$ \\
ID end plate & 54 & 344 & $2.4 \times 10^{11}$ & 48 & $1.02 \times 10^{11}$ \\
ID end plate & 80 & 344 & $1.5 \times 10^{11}$ & 23 & $0.87 \times 10^{11}$ \\
Cryostat wall & 110 & 1 & $0.46 \times 10^{11}$ & 5.1 & $0.40 \times 10^{11}$ \\
\hline
\end{tabular}

predicted, differences were typically less than $30 \%$ [55]. Table 9 shows the corresponding FLUKA predictions for $1 \mathrm{MeV}$ neutron-equivalent fluence, ionising dose and thermal neutron fluence at the positions of the four radiation monitors, the locations of which can be seen in figure 28 .

\subsection{Detector leakage currents}

The increase in detector leakage current is proportional to the $1 \mathrm{MeV}$ neutron-equivalent fluence and is sensitive to temperature and annealing effects. Leakage-current predictions are obtained from two models, the Hamburg/Dortmund model [56, 57] and the Harper model [58], each using different assumptions for the temperature-dependent annealing behaviour (see appendix A for details). Both models include self-annealing effects with various time constants. As a consequence, the history of sensor temperature must be carefully tracked including warm-up periods, even if they are short, and this is described in section 7.2.1. The sensor temperature during the periods without cooling was assumed to be the same as the enviromental gas temperature, which was $17.5^{\circ} \mathrm{C}$ during the 2011-2012 winter shutdown. 
The largest uncertainty in the leakage-current predictions comes from the $1 \mathrm{MeV}$ neutronequivalent fluence simulation, so the comparison of measurement with prediction is an important check of the fluence simulations.

\subsubsection{Temperature and leakage-current measurement}

The measured leakage-current values $I_{\mathrm{HV}}$ are normalised to those at a temperature of $0^{\circ} \mathrm{C}, I_{\text {norm }}$, using the temperature scaling formula for the silicon bulk generation current:

$$
\left(\frac{I_{\text {norm }}}{I_{\mathrm{HV}}}\right)=\left(\frac{T_{\text {norm }}}{T_{\text {meas }}}\right)^{2} \exp \left[-\frac{E_{\text {gen }}}{2 k_{\mathrm{B}}}\left(\frac{1}{T_{\text {norm }}}-\frac{1}{T_{\text {meas }}}\right)\right]
$$

where $T_{\text {meas }}\left(T_{\text {norm }}\right)$ is the sensor (normalisation) temperature, $E_{\text {gen }}$ the effective generation energy of $1.21 \mathrm{eV}$ [59] and $k_{\mathrm{B}}$ is Boltzmann's constant. The sensor temperature is deduced from the temperature measured by thermistors mounted on the hybrid circuits. For the barrel modules, FEM thermal simulations predict the average sensor temperature to be $3.7 \pm 1.0^{\circ} \mathrm{C}$ below the thermistor temperature, and to vary by only $\sim 0.2^{\circ} \mathrm{C}$ across the sensor. Predicted temperature differences for the endcap sensors have large uncertainties. Furthermore a systematic difference in the raw leakage current between the two sides $\mathrm{A}$ and $\mathrm{C}$ exists, the reason for which is currently under investigation. Therefore results are presented below for the barrel only.

Figure 29 shows module-by-module distributions of high voltage, hybrid temperature and raw leakage current per module as well as the normalised leakage current per unit volume at $0^{\circ} \mathrm{C}$ for the innermost barrel of the SCT. Periodic increases in raw leakage current, which arise from higher temperatures in one cooling loop, disappear after temperature normalisation, resulting in a distribution which is quite flat along $z$, a reflection of the flat pseudorapidity distribution of secondary particles in minimum-bias events. Similar flat distributions are seen in all other barrel layers.

\subsubsection{Leakage-current evolution and comparison with predictions}

In 2010 and 2011, when the leakage currents were small, electrical noise from the FSI alignment system interfered with the measurements, and the study of the leakage currents was limited to periods when the FSI system was off. No such restrictions were necessary in 2012.

Figure 30 shows the average leakage current of each barrel layer compared with the predictions of the Hamburg/Dortmund model [56, 57]. The coloured bands indicate $1 \sigma$ uncertainties on the model predictions. They were calculated by varying each parameter of the model by $\pm 1 \sigma$ and then adding the deviations in quadrature. Uncertainties on temperature measurements $\left( \pm 1^{\circ} \mathrm{C}\right)$ and delivered luminosities $( \pm 3.7 \%)$ are also taken into account; the uncertainty on the FLUKA fluence simulations is not included. The Harper model [58] predicts quite similar profiles but with about $15 \%$ larger values systematically. The total uncertainties on the model predictions are at the level of $\pm 20 \%$. The major uncertainties come from two parameters $\left(k_{0 \mathrm{I}}\right.$ and $\left.E_{\mathrm{I}}\right)$ in the Hamburg/Dortmund model (see appendix A for details), but from one current-damage constant $\left(A_{1}\right)$ with an annealing time of 833 days at $-7^{\circ} \mathrm{C}$ in the Harper model. For all barrel layers, the data are within the uncertainties of the model predictions over a period of 2.5 years and four orders of magnitude in leakage current. This strongly suggests that the radiation fluences are well described by the FLUKA predictions. 


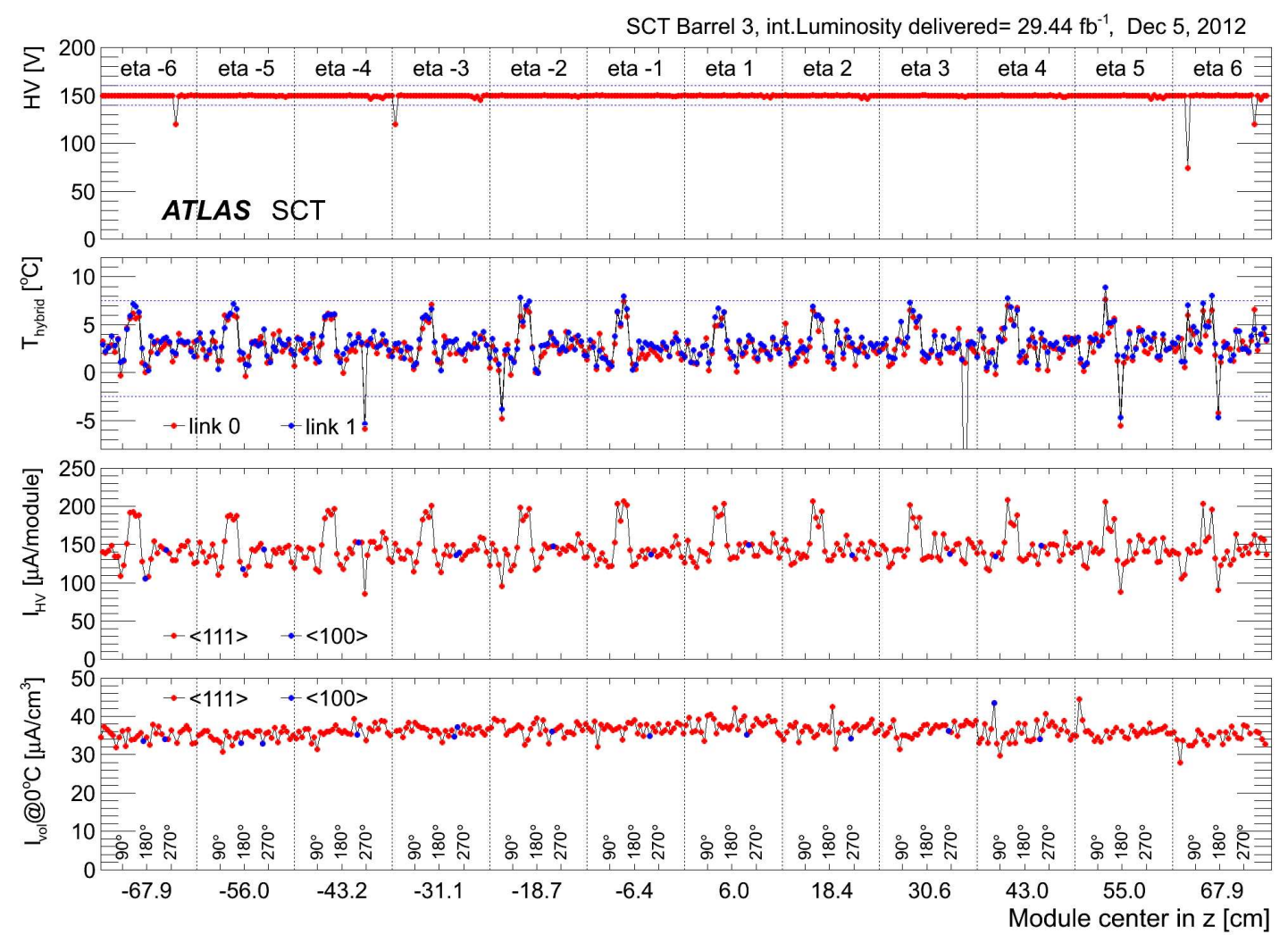

Figure 29. From top to bottom: high voltage (HV), hybrid temperature $\left(T_{\text {hybrid }}\right)$, measured leakage current $\left(I_{\mathrm{HV}}\right)$ and normalised leakage current per unit volume at $0^{\circ} \mathrm{C}\left(I_{\mathrm{vol}}\right)$ for each module of barrel 3 as of December 2012. The temperature of each module is measured by two thermistors; values from those mounted on the inner (outer) sides of modules are shown in blue (red).

\subsection{Online radiation monitor measurements}

In addition to the SCT leakage-current measurements, the online radiation monitoring system also offers important information on fluences and doses in the inner detector volume. Details can be found in refs. $[60,61]$ and only a brief description is given here. The radiation-monitor packages provide measurements of non-ionising energy loss (NIEL), total ionising dose (TID) and thermal neutron fluence. NIEL is monitored using $p-i-n$ diodes and two methods are employed. The first measures the increase in the forward voltage for a given forward current and is used for determining NIEL in the fluence range $10^{9}$ to $5 \cdot 10^{12} \mathrm{n} / \mathrm{cm}^{2}$. This method exploits the increase in silicon resistivity from radiation due to degradation in minority-carrier lifetime. The second method measures the increase in leakage current in a way similar to the SCT silicon sensors. TID is measured with radiation-sensitive p-MOSFET transistors (RadFETs). Ionising radiation creates electron-hole pairs in the silicon oxide; positive charge is trapped in the gate oxide, and an increasing negative gate voltage must be applied for a given drain current. The increase in gate voltage provides a measure of the total ionising dose. The thickness of the oxide layer defines the sensitivity and range of doses that can be measured and different RadFETs are used to cover the required dynamic range. Measurements of TID are of particular interest for predicting degradation of the SCT front- 


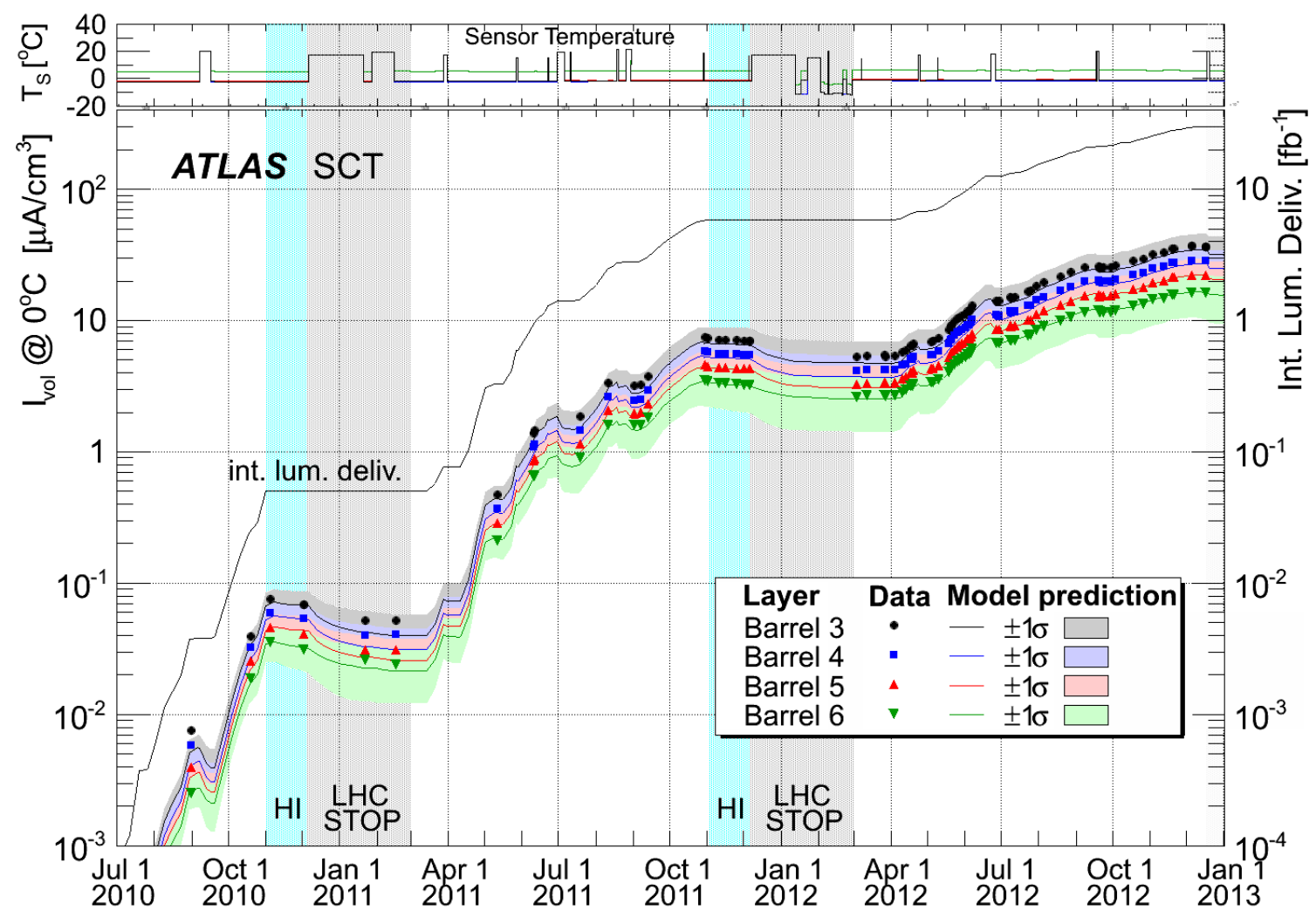

Figure 30. Comparison between data (points) and Hamburg/Dortmund model predictions (lines with uncertainties shown by the coloured bands) of the leakage current per unit volume at $0^{\circ} \mathrm{C}\left(I_{\mathrm{vol}}\right)$ of the four barrel layers. The integrated luminosity and the average sensor temperatures are also shown. The blue shading and label 'HI' indicate periods of heavy-ion running, while extended periods with no beam in the LHC during which the SCT was off are shaded grey.

end chips, and the radiation monitors provide the only online information about TID near to the SCT. Thermal neutron fluence is estimated from the decrease in common-emitter-current gain of $n-p-n$ bipolar transistors, which are the same as those used in the SCT read-out chips. The gain in these transistors is degraded due to bulk damage caused by fast hadrons and by thermal neutrons. Since the $1 \mathrm{MeV}$ equivalent fluence of fast hadrons is known from measurements with diodes, the contribution of thermal neutrons can be estimated from the gain degradation [60].

The measured voltages and leakage currents are converted to fluences and doses using parameterisations based on calibrated irradiation data [60]. Figure 31 shows measurements of TID and NIEL at the four sets of locations shown in figure 28 and listed in table 9. The NIEL measurements for the pixel support tube and inner detector end-plate locations are obtained from the reverse current increase, whereas the values for the cryostat wall are obtained using the forward bias method. The measurements are average values from sensors at the same $r$ and $|z|$ placed at different azimuth angles $\phi$. The shaded bands represent the r.m.s. of the measurements with a $20 \%$ systematic error added in quadrature. The systematic error is dominated by the sensor calibration precision from the irradiations and the measured sensor temperature. The predicted fluence and dose are shown as dotted lines and are obtained by multiplying the values from table 9 by the delivered integrated 
luminosity at a certain date. Very good agreement between measurement and simulation can be seen, especially for TID.

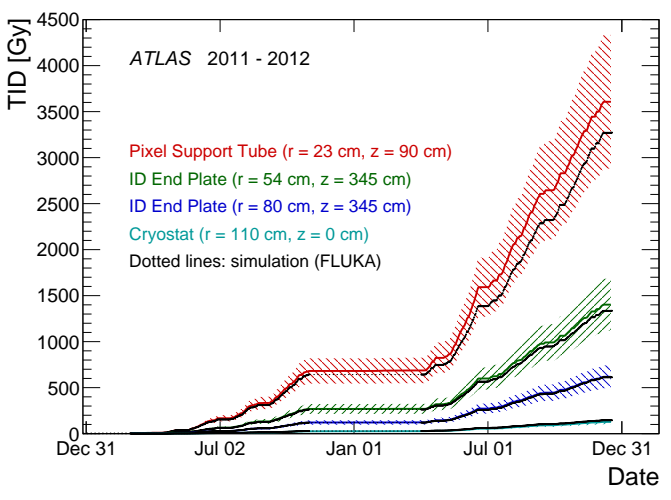

(a)

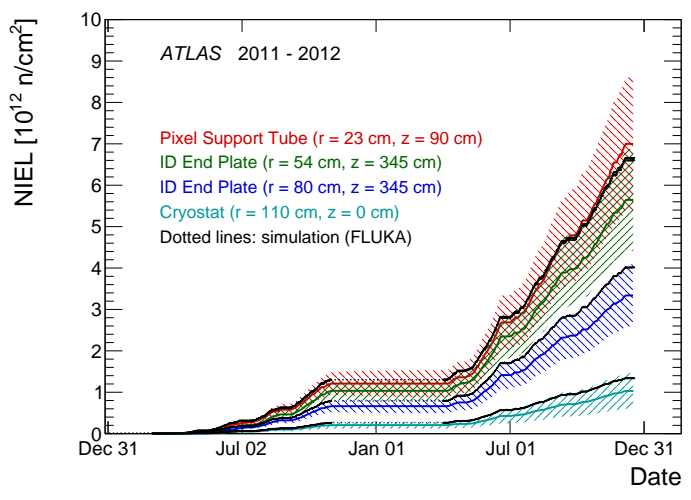

(b)

Figure 31. (a) Total ionising dose (TID) and (b) non-ionising energy loss (NIEL) in $1 \mathrm{MeV}$ neutronequivalent fluence measured at four different sets of locations in the inner detector. Each location is drawn with a different colour. Coloured bands represent measurement uncertainties. The simulated values at each location are shown with black dotted lines. The simulated values of NIEL for the pixel support tube and ID end plate $(r=54 \mathrm{~cm})$ are very similar.

The base current in transistors increases with increasing integrated luminosity. At the end of 2012 it has increased by about $10 \mathrm{nA}$ from an initial value of $70 \mathrm{nA}$ (at $10 \mu \mathrm{A}$ collector current) in the most exposed transistors on the pixel support tube and inner detector end-plates. This increase is consistent with a thermal neutron fluence of the order of $10^{12} \mathrm{n} / \mathrm{cm}^{2}$, which is in agreement with the FLUKA calculations in table 9.

\subsection{Single-event upsets}

As well as causing damage to the sensors and on-detector readout electronics, radiation can also cause 'soft errors', or single-event upsets (SEUs), in the SCT on-detector electronics. SEUs can induce bit flips in the static registers of the ABCD chips, and can also affect the on-detector $p-i-n$ diodes. In both cases clear SEU signals have been identified in data from the barrel modules, as summarised below; more details can be found in ref. [62].

The registers in the ABCD chips are not protected against SEU, and test-beam studies have measured a small but non-zero SEU cross-section [63]. In the SCT, a direct read-back of register values is not possible, so the analysis of SEU rates uses the sudden increase in occupancy from individual chips which can arise when a bit is flipped in the threshold registers. In particular, the threshold registers use an 8-bit DAC, with the fifth bit usually set to one. When this bit is flipped, the chip outputs the maximum number of strips (128) for each trigger. A few problematic chips which gave spurious SEU-like noise bursts were removed from the analysis. A verification of the SEU hypothesis is provided by studying the correlation between SEU rate and module occupancy. Figure 32a shows the SEU rate as a function of cluster occupancy in a module; occupancy is proportional to flux. The data show the expected linear behaviour. The apparent saturation observed 
at high occupancy partially arises from the requirement in the analysis that there be at most one SEU per chip per run.

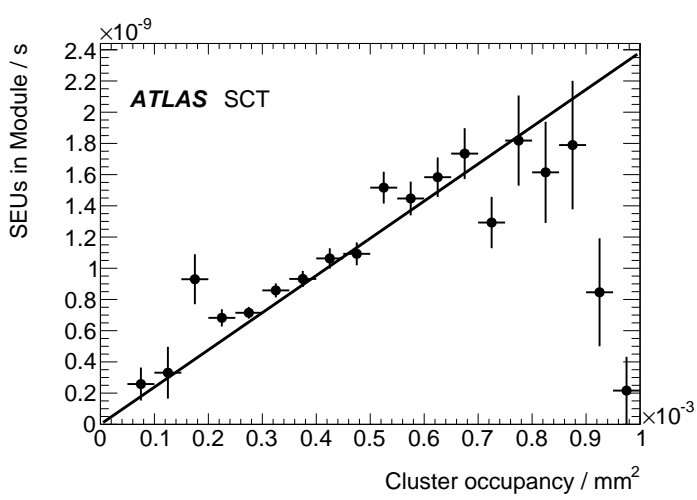

(a)

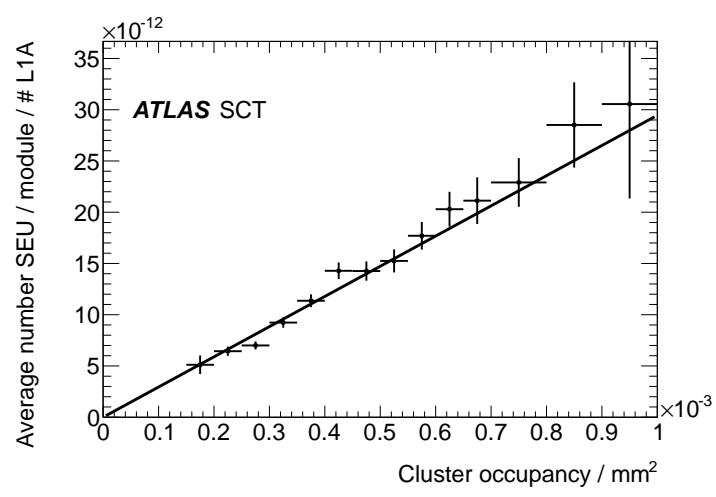

(b)

Figure 32. (a) SEU rate in the ABCD DAC register versus module cluster occupancy in a run. (b) Mean number of SEUs per module per level-1 trigger (L1A) in a run versus cluster occupancy in that module and run. Note that in each case same module contributes to several bins depending on the cluster occupancy recorded in different runs. The lines show the results of linear fits through the origin.

Another measurable effect of SEUs is the desynchronisation of individual modules from the rest of the ATLAS readout. Triggers are transmitted to the modules via optical links, and testbeam studies have demonstrated that an SEU arising from a relatively small energy deposition in the receiving $p-i-n$ diode can cause a bit flip in a discriminator [64] and may lead to a missing trigger. Desynchronisation is flagged when the trigger count in the ROD differs from the trigger count received from the module, and the mismatch persists until the module is reset. Figure $32 \mathrm{~b}$ shows the average number of SEUs per module per level-1 trigger in a run versus cluster occupancy for that module in the run. This shows the expected linear trend, confirming that these errors are genuine SEUs. Further confirmation of the SEUs hypothesis comes from the observation that the number of SEUs in a module correlates with decreasing $p-i-n$ diode current, as expected and measured in the test-beam studies [64].

\subsection{Impact of radiation on detector operation and its mitigation}

The SCT power-supply system incorporates adjustable trip limits for various parameters which are set to ensure that faults are quickly identified and addressed. Most parameters are stable, but as described in section 7.2, significant increases in leakage currents were observed in the SCT throughout 2011 and 2012. Throughout 2010, module trip limits were maintained at $5 \mu \mathrm{A}$, which was comfortably above leakage currents for unirradiated modules, but low enough to flag powersupply issues. The limits were increased to $50 \mu \mathrm{A}$ and then $100 \mu \mathrm{A}$ in 2011 and 2012 respectively, to track the evolution of the current.

During running early in 2012 a significant fraction (27\%) of the modules with CiSmanufactured sensors started to exhibit anomalously high and varying leakage currents after a few hours of proton-proton collisions. Figure 33a shows the leakage-current profile with time for 


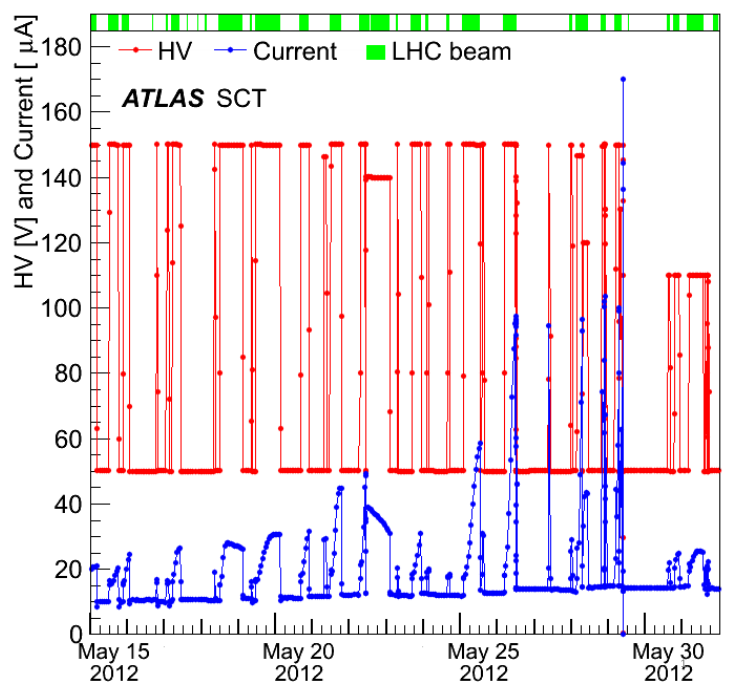

(a)

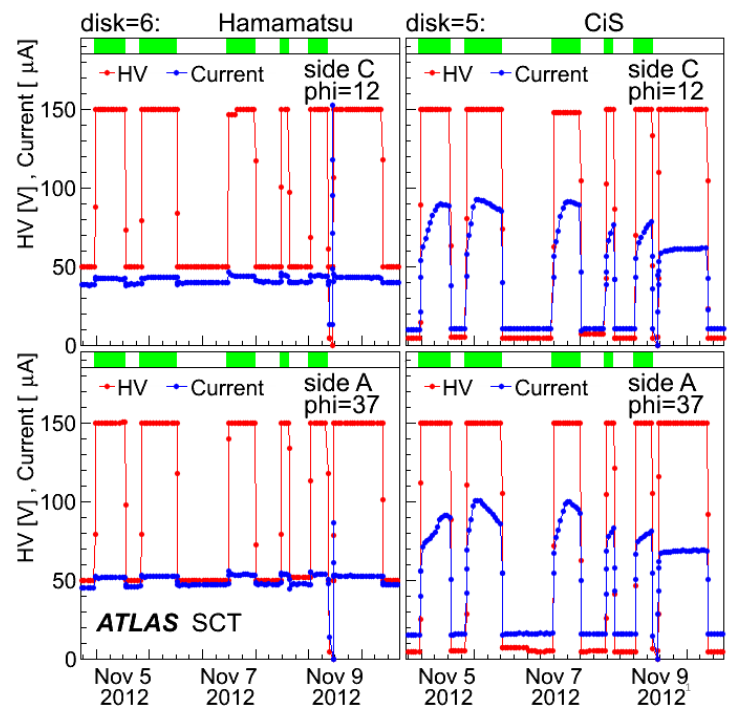

(b)

Figure 33. (a) Leakage current (blue) observed in May 2012 together with high voltage (red) for one endcap module showing anomalous behaviour. (b) Leakage current (blue) and high voltage (red), observed in November 2012, of typical endcap modules with sensors manufactured by Hamamatsu (left) and CiS (right). Periods with LHC colliding beams are indicated by the green bands at the top of the figure in each case.

one such module, illustrating the leakage current with the module at standby $(50 \mathrm{~V})$ and fully on $(150 \mathrm{~V})$. In this example the current increases significantly during the course of each run; at the same time there was an abnormally high increase in noise sufficient to provoke a ROD busy signal and prevent data taking. The behaviour was mitigated by reducing the HV by typically $30-40 \mathrm{~V}$ specifically for the modules associated with the high noise; for those modules the HV still remained above the highest depletion voltage of the sensors within the module so that hit efficiency was not impacted.

Empirically it was found that setting all CiS modules to $5 \mathrm{~V}$ during standby (instead of $50 \mathrm{~V}$ ) prevented the more severe current increases associated with the noise problems, though the current profile during the run still varied widely. The modules which had previously provoked ROD busy signals continued to be operated with the reduced HV. Figure $33 \mathrm{~b}$ shows typical beam-time behaviours of HV and current in late 2012. Modules with Hamamatsu sensors (left) show the expected flat current profiles while those with CiS sensors (right) exhibit varying current behaviours during beam time. The last run in these plots corresponds to a day-long calibration run with no beam and all currents stayed constant. The cause of the current anomalies has not been understood, though it is clearly correlated with the presence of radiation. The behaviour may evolve as the sensors experience further radiation damage in future.

The online monitoring flags very noisy modules caused by SEUs in the ABCD registers, allowing shifters to do prompt resets. Even if a shifter fails to respond, the regular module resets limit the data loss to a tolerable level (typically the loss of one chip for up to 30 minutes). The 
DAQ detects trigger synchronisation errors and resets the counters and pipelines for the module with errors. This procedure takes about $30 \mathrm{~s}$ and therefore reduces the data loss resulting from SEUs in the $p-i-n$ diodes to a negligible level, as illustrated in figure 7.

\section{Conclusions}

The operation and performance of the ATLAS semiconductor tracker during 2009-2013 are described in this paper. During this period, more than $99 \%$ of detector modules were operational, and more than $99 \%$ of data collected by the ATLAS experiment had good SCT data quality. The frequency-scannning interferometry system showed the position of the detector to be stable at the micron level over long periods of time. Measurements of the increase in leakage currents with time are consistent with the radiation-damage predictions. The differences between data and simulation are typically less than $30 \%$. This level of agreement exceeds expectations, and provides confidence in the fluence predictions. The verification of the simulations will be repeated at higher beam energies in future. Single event upsets have been identified and measured in the barrel module data, and a strategy for their mitigation implemented.

The detector occupancy was found to vary linearly with the number of interactions per bunch crossing, up to the maximum of 70 interactions per crossing, where it is less than $2 \%$ in the innermost barrel layer (which has highest occupancy). The intrinsic hit efficiency of the detector was measured to be $(99.74 \pm 0.04) \%$, and the noise occupancies of almost all chips remained below the design requirement of $5 \times 10^{-4}$.

Measured values of the Lorentz angle are compatible with model predictions within at most twice the estimated uncertainties on those predictions. The measured values for sensors with $<100>$ crystal orientation are approximately $1^{\circ}$ lower than for those with $<111>$ crystal orientation, contrary to the expectation that a higher expected charge-carrier mobility in the sensors with $<100>$ crystal orientation should result in a higher value of the Lorentz angle.

Despite the binary readout, some particle identification from energy-loss measurements is possible: the discriminating power arises from the number of time bins above threshold and cluster widths. The position of the proton energy-loss peak was found to be stable at the 5-10\% level during 2010-2012. The position of this peak may become a useful tool for monitoring radiation damage in future. The production of $\delta$-rays in the silicon sensors was measured, and is found to be in good agreement with expectations.

\section{Acknowledgments}

We thank CERN for the very successful operation of the LHC, as well as the support staff from our institutions without whom ATLAS could not be operated efficiently.

We acknowledge the support of ANPCyT, Argentina; YerPhI, Armenia; ARC, Australia; BMWF and FWF, Austria; ANAS, Azerbaijan; SSTC, Belarus; CNPq and FAPESP, Brazil; NSERC, NRC and CFI, Canada; CERN; CONICYT, Chile; CAS, MOST and NSFC, China; COLCIENCIAS, Colombia; MSMT CR, MPO CR and VSC CR, Czech Republic; DNRF, DNSRC and Lundbeck Foundation, Denmark; EPLANET, ERC and NSRF, European Union; IN2P3-CNRS, 
CEA-DSM/IRFU, France; GNSF, Georgia; BMBF, DFG, HGF, MPG and AvH Foundation, Germany; GSRT and NSRF, Greece; ISF, MINERVA, GIF, I-CORE and Benoziyo Center, Israel; INFN, Italy; MEXT and JSPS, Japan; CNRST, Morocco; FOM and NWO, Netherlands; BRF and RCN, Norway; MNiSW and NCN, Poland; GRICES and FCT, Portugal; MNE/IFA, Romania; MES of Russia and ROSATOM, Russian Federation; JINR; MSTD, Serbia; MSSR, Slovakia; ARRS and MIZŠ, Slovenia; DST/NRF, South Africa; MINECO, Spain; SRC and Wallenberg Foundation, Sweden; SER, SNSF and Cantons of Bern and Geneva, Switzerland; NSC, Taiwan; TAEK, Turkey; STFC, the Royal Society and Leverhulme Trust, United Kingdom; DOE and NSF, United States of America.

The crucial computing support from all WLCG partners is acknowledged gratefully, in particular from CERN and the ATLAS Tier-1 facilities at TRIUMF (Canada), NDGF (Denmark, Norway, Sweden), CC-IN2P3 (France), KIT/GridKA (Germany), INFN-CNAF (Italy), NL-T1 (Netherlands), PIC (Spain), ASGC (Taiwan), RAL (U.K.) and BNL (U.S.A.) and in the Tier-2 facilities worldwide.

\section{A Estimation of the bulk leakage current using models}

It is assumed that the whole period from the beginning of the experiment to the present time is divided into $n$ time segments. During each time segment with a time interval of $\delta t_{i}[\mathrm{~s}]$, a sensor at a bulk temperature of $T_{i}$ is supposed to receive a $1 \mathrm{MeV}$ neutron-equivalent fluence of $\delta \Phi_{i}^{\mathrm{eq}}\left[\mathrm{cm}^{-2}\right]$. The prediction of the leakage current $I_{n}\left(T_{\text {ref }}\right)\left[\mathrm{A} / \mathrm{cm}^{3}\right]$ at the end of time segment $n$ can be calculated using the annealing formulae given below, where $T_{\text {ref }}$ is the reference temperature used in the model. For the comparison with data, the temperature scaling in equation (7.1) is used to convert the predicted values to those at $0^{\circ} \mathrm{C}$.

\section{Hamburg/Dortmund model:}

the Hamburg/Dortmund model for the bulk leakage current is based on the work by M. Moll [56] and O. Krasel [57].

$$
\begin{array}{rlrl}
I_{n}\left(T_{\mathrm{ref}}\right) & \equiv I_{n}^{\mathrm{exp}}+I_{n}^{\mathrm{log}}=\sum_{i=1}^{n} \delta \Phi_{i}^{\mathrm{eq}} \cdot \alpha_{\mathrm{I}} \cdot \exp \left(-\frac{t_{n, i}^{\mathrm{I}}}{\tau_{\mathrm{I}}\left(T_{\mathrm{ref}}\right)}\right)+\sum_{i=1}^{n} \delta \Phi_{i}^{\mathrm{eq}}\left(\alpha_{0}^{*}-\beta \log \left(t_{n, i}^{\log } / t_{0}\right)\right) \\
t_{n, i}^{\mathrm{I}} & =\sum_{j=i}^{n} \delta t_{j} \frac{\tau_{\mathrm{I}}\left(T_{\mathrm{ref}}\right)}{\tau_{\mathrm{I}}\left(T_{j}\right)}, & t_{n, i}^{\log }=\sum_{j=i}^{n} \delta t_{j} \Theta_{\mathrm{A}}\left(T_{j}\right) \\
\frac{1}{\tau_{\mathrm{I}}(T)}=k_{0 \mathrm{I}} \cdot \exp \left(-\frac{E_{\mathrm{I}}}{k_{\mathrm{B}} T}\right), & \Theta_{\mathrm{A}}(T)=\exp \left(-\frac{E_{\mathrm{I}}^{*}}{k_{\mathrm{B}}}\left[\frac{1}{T}-\frac{1}{T_{\mathrm{ref}}}\right]\right)
\end{array}
$$

where $k_{\mathrm{B}}$ is Boltzmann's constant, $\alpha_{\mathrm{I}}=(1.23 \pm 0.06) \times 10^{-17} \mathrm{~A} / \mathrm{cm}, k_{0 \mathrm{I}}=1.2_{-1.0}^{+5.3} \times 10^{13} \mathrm{~s}^{-1}$, $E_{\mathrm{I}}=(1.11 \pm 0.03) \mathrm{eV}, \alpha_{0}^{*}=7.07 \times 10^{-17} \mathrm{~A} / \mathrm{cm}, \beta=3.29 \times 10^{-18} \mathrm{~A} / \mathrm{cm}, E_{\mathrm{I}}^{*}=(1.30 \pm$ $0.14) \mathrm{eV}, T_{\text {ref }}=21^{\circ} \mathrm{C}$ and $t_{0}=1 \mathrm{~min}$. The uncertainties on $\alpha_{0}^{*}$ and $\beta$ cannot be found in the references and are set to $\pm 10 \%$. 
2. Harper model:

the Harper model for the bulk leakage current is based on the work by R. Harper [58].

$$
\begin{aligned}
I_{n}\left(T_{\mathrm{ref}}\right) & \equiv \sum_{i=1}^{n} g_{n, i} \cdot \alpha\left(T_{\mathrm{ref}}\right) \cdot \delta \Phi_{i}^{\mathrm{eq}} \\
g_{n, i} & =\sum_{k=1}^{5}\left\{A_{k} \frac{\tau_{k}}{\Theta_{\mathrm{A}}\left(T_{i}\right) \delta t_{i}}\left[1-\exp \left(\frac{-\Theta_{\mathrm{A}}\left(T_{i}\right) \delta t_{i}}{\tau_{k}}\right)\right] \exp \left(-\frac{1}{\tau_{k}} \sum_{j=i+1}^{n} \Theta_{\mathrm{A}}\left(T_{j}\right) \delta t_{j}\right)\right\}
\end{aligned}
$$

where $\alpha\left(T_{\text {ref }}=20^{\circ} \mathrm{C}\right)=(4.81 \pm 0.13) \times 10^{-17} \mathrm{~A} / \mathrm{cm}$ is the current-related damage constant. The annealing effects are divided into five different components ( $k=1$ to 5 ) with annealing time constants of $\tau_{k}$ and amplitudes $A_{k}$ as given in table 10. $\Theta_{\mathrm{A}}(T)$ is the Arrhenius function used to scale the annealing time. It gives the annealing rate at a temperature $T$ relative to the rate at the reference temperature of $T_{\text {ref }}$ :

$$
\Theta_{\mathrm{A}}(T)=\exp \left(\frac{E_{\mathrm{I}}}{k_{\mathrm{B}}}\left[\frac{1}{T_{\text {ref }}}-\frac{1}{T}\right]\right), \quad E_{\mathrm{I}}=1.09 \pm 0.14 \mathrm{eV} .
$$

Table 10. Annealing time constants and amplitudes at $T_{\text {ref }}=20^{\circ} \mathrm{C}$ of the Harper model.

\begin{tabular}{|ccc|}
\hline$k$ & $\tau_{k}[\mathrm{~min}]$ & $A_{k}$ \\
\hline 1 & $(1.2 \pm 0.2) \times 10^{6}$ & $0.42 \pm 0.11$ \\
2 & $(4.1 \pm 0.6) \times 10^{4}$ & $0.10 \pm 0.01$ \\
3 & $(3.7 \pm 0.3) \times 10^{3}$ & $0.23 \pm 0.02$ \\
4 & $124 \pm 2.5$ & $0.21 \pm 0.02$ \\
5 & $8 \pm 5$ & $0.04 \pm 0.03$ \\
\hline
\end{tabular}

\section{References}

[1] ATLAS collaboration, The ATLAS Experiment at the CERN Large Hadron Collider, 2008 JINST 3 S08003.

[2] L. Evans and P. Bryant, LHC Machine, 2008 JINST 3 S08001.

[3] ATLAS collaboration, Observation of a new particle in the search for the Standard Model Higgs boson with the ATLAS detector at the LHC, Phys. Lett. B 716 (2012) 1 [arXiv:1207.7214].

[4] CMS collaboration, Observation of a new boson at a mass of $125 \mathrm{GeV}$ with the CMS experiment at the LHC, Phys. Lett. B 716 (2012) 30 [arXiv:1207.7235].

[5] ATLAS collaboration, The ATLAS Inner Detector commissioning and calibration, Eur. Phys. J. C 70 (2010) 787 [arXiv: 1004 .5293].

[6] ATLAS collaboration, Improved luminosity determination in pp collisions at $\sqrt{s}=7 \mathrm{TeV}$ using the ATLAS detector at the LHC, Eur. Phys. J. C 73 (2013) 2518 [arXiv: 1302 .4393].

[7] A. Abdesselam et al., The barrel modules of the ATLAS semiconductor tracker, Nucl. Instrum. Meth. A $568(2006) 642$. 
[8] A. Ahmad et al., The Silicon microstrip sensors of the ATLAS semiconductor tracker, Nucl. Instrum. Meth. A 578 (2007) 98.

[9] A. Abdesselam et al., The ATLAS semiconductor tracker end-cap module, Nucl. Instrum. Meth. A 575 (2007) 353.

[10] F. Campabadal et al., Design and performance of the ABCD3TA ASIC for readout of silicon strip detectors in the ATLAS semiconductor tracker, Nucl. Instrum. Meth. A 552 (2005) 292.

[11] A. Abdesselam et al., The Data acquisition and calibration system for the ATLAS semiconductor tracker, 2008 JINST 3 P01003.

[12] A. Abdesselam et al., The optical links of the ATLAS SemiConductor tracker, 2007 JINST 2 P09003.

[13] D. Attree et al., The evaporative cooling system for the ATLAS inner detector, 2008 JINST 3 P07003.

[14] A. Abdesselam et al., The detector control system of the ATLAS SemiConductor tracker during macro-assembly and integration, 2008 JINST 3 P02007.

[15] A. Barriuso Poy et al., The detector control system of the ATLAS experiment, 2008 JINST 3 P05006.

[16] B. Hallgren, H. Boterenbrood, H.J. Burckhart and H. Kvedalen, The embedded local monitor board (ELMB) in the LHC front-end I/O control system, in proceedings of $7^{\text {th }}$ workshop on Electronics for LHC Experiments, Stockholm, Sweden (2001), pg. 325, http://cdsweb.cern.ch/record/530675.

[17] R.L. Bates et al., The ATLAS SCT grounding and shielding concept and implementation, 2012 JINST 7 P03005.

[18] S.M.Gibson et al. on behalf of the ATLAS SCT collaboration, A novel method for ATLAS FSI alignment based on rapid, direct phase monitoring, ATL-INDET-PROC-2010-037 (2010).

[19] O. Beltramello et al., The detector safety system of the ATLAS experiment, 2009 JINST 4 P09012.

[20] D. Dobos and H. Pernegger on behalf of the RD42, ATLAS DiAmOND PIXEL UPGRADE and ATLAS BEAM CONDITIONS MONITOR collaborations, Diamond pixel modules and the ATLAS beam conditions monitor, Nucl. Instrum. Meth. A 628 (2011) 246.

[21] V. Cindro et al., The ATLAS beam conditions monitor, 2008 JINST 3 P02004.

[22] B. Maček on behalf of the ATLAS BCM/BLM group, Performance of the ATLAS Beam Diagnostic Systems, IEEE Nucl. Sci. Symp. Conf. Rec. 2010 (2010) 1565.

[23] A.R.Weidberg on behalf of the ATLAS collaboration, VCSEL reliability in ATLAS and development of robust arrays, 2012 JINST 7 C01098.

[24] ATLAS collaboration, The Athena Framework, in ATLAS Computing: technical design report, pg. 27, CERN-LHCC-2005-022.

[25] T. Cornelissen et al., Concepts, Design and Implementation of the ATLAS New Tracking (NEWT), ATL-SOFT-PUB-2007-007 (2007).

[26] T.G. Cornelissen et al., The global $\chi^{2}$ track fitter in ATLAS, J. Phys. Conf. Ser. 119 (2008) 032013.

[27] T. Sjöstrand, S. Mrenna and P.Z. Skands, A Brief Introduction to PYTHIA 8.1, Comput. Phys. Commun. 178 (2008) 852 [arXiv: 0710.3820].

[28] ATLAS collaboration, Summary of ATLAS PYTHIA 8 tunes, ATL-PHYS-PUB-2012-003 (2012).

[29] ATLAS collaboration, Alignment requirements, ATLAS Inner Detector Design Report, vol.I, CERN-LHCC-1997-0016. 
[30] ATLAS collaboration, Alignment Performance of the ATLAS Inner Detector Tracking System in 7 TeV proton-proton collisions at the LHC, ATLAS-CONF-2010-067 (2010).

[31] S.-C.Hsu on behalf of the ATLAS collaboration, Alignment of the ATLAS inner detector tracking system, Nucl. Phys. Proc. Suppl. 215 (2011) 92.

[32] GEANT4 collaboration, S. Agostinelli et al., GEANT4 - A Simulation toolkit, Nucl. Instrum. Meth. A 506 (2003) 250.

[33] ATLAS collaboration, The ATLAS Simulation Infrastructure, Eur. Phys. J. C 70 (2010) 823 [arXiv: 1005.4568].

[34] S. Ramo, Currents induced by electron motion, Proc. Ire. 27 (1939) 584.

[35] V. Radeka, Low Noise Techniques in Detectors, Ann. Rev. Nucl. Part. Sci. 38 (1988) 217.

[36] G. Cavalleri, E. Gatti, G. Fabri and V. Svelto, Extension of Ramo's theorem as applied to induced charge in semiconductor detectors, Nucl. Instrum. Meth. 92 (1971) 137.

[37] A. Valassi et al., COOL, LCG Conditions Database for the LHC Experiments: Development and Deployment Status, IEEE Nucl. Sci. Symp. Conf. Rec. 2008 (2008) 3021.

[38] S. Kolos, A. Corso-Radu, H. Hadavand, M. Hauschild and R. Kehoe, A software framework for Data Quality Monitoring in ATLAS, J. Phys. Conf. Ser. 119 (2008) 022033.

[39] C. Cuenca Almenar et al., ATLAS online data quality monitoring, Nucl. Phys. Proc. Suppl. 215 (2011) 304.

[40] J. Adelman et al., ATLAS offline data quality monitoring, J. Phys. Conf. Ser. 219 (2010) 042018.

[41] T. Golling, H.S. Hayward, P.U.E. Onyisi, H.J. Stelzer and P. Waller, The ATLAS Data Quality Defect Database System, Eur. Phys. J. C 72 (2012) 1960 [arXiv: 1110.6119].

[42] Y. Unno et al., Beamtest of nonirradiated and irradiated ATLAS SCT microstrip modules at KEK, IEEE Trans. Nucl. Sci. 49 (2002) 1868.

[43] F. Campabadal et al., Beam tests of ATLAS SCT silicon strip detector modules, Nucl. Instrum. Meth. A 538 (2005) 384.

[44] C. Jacoboni, C. Canali, G. Ottaviani and A. Alberigi Quaranta, A review of some charge transport properties of silicon, Solid-State Electronics 20 (1977) 77.

[45] J. Becker, E. Fretwurst and R. Klanner, Measurements of charge carrier mobilities and drift velocity saturation in bulk silicon of $\langle 111>$ and $<100>$ crystal orientation at high electric fields, Solid-State Electronics 56 (2011) 104.

[46] ATLAS collaboration, $d E / d x$ measurement in the ATLAS Pixel Detector and its use for particle identification, ATLAS-CONF-2011-016 (2011).

[47] A. Ferrari, P.R. Sala, A. Fasso and J. Ranft, FLUKA: A multi-particle transport code (Program version 2005), CERN-2005-010, SLAC-R-773, INFN-TC-05-11.

[48] G. Battistoni et al., The FLUKA code: description and benchmarking, AIP Conf. Proc. 896 (2007) 31.

[49] S. Baranov, M. Bosman, I. Dawson, V. Hedberg, A. Nisati and M. Shupe, Estimation of Radiation Background, Impact on Detectors, Activation and Shielding Optimization in ATLAS, ATL-GEN-2005-001 (2005).

[50] R. Engel, Photoproduction within the two component dual parton model. 1. Amplitudes and cross-sections, Z. Phys. C 66 (1995) 203. 
[51] ATLAS collaboration, Measurement of the Inelastic Proton-Proton Cross-Section at $\sqrt{s}=7 \mathrm{TeV}$ with the ATLAS Detector, Nature Commun. 2 (2011) 463 [arXiv: 1104.0326].

[52] CMS collaboration, Measurement of the inelastic proton-proton cross section at $\sqrt{s}=7 \mathrm{TeV}$, Phys. Lett. B 722 (2013) 5 [arXiv:1210.6718].

[53] TOTEM collaboration, G.Antchev et al., First measurement of the total proton-proton cross section at the LHC energy of $\sqrt{s}=7$ TeV, Europhys. Lett. 96 (2011) 21002 [arXiv:1110.1395].

[54] ALICE collaboration, B. Abelev et al., Measurement of inelastic, single-and double-diffraction cross sections in proton-proton collisions at the LHC with ALICE, Eur. Phys. J. C 73 (2013) 2456 [arXiv: 1208.4968].

[55] I. Dawson on behalf of the ATLAS INNER DETECTOR, Radiation background simulation and verification at the LHC: Examples from the ATLAS experiment and its upgrades, PoS (Vertex 2012) 015.

[56] M. Moll, Radiation Damage in Silicon Particle Detectors, Thesis, Universitität Hamburg, Germany (1999), DESY-THESIS-1999-040.

[57] O. Krasel, Charge Collection in Irradiated Silicon-Detectors, Thesis, Universitität Dortmund, Germany (2004), http://hdl.handle.net/2003/2354.

[58] R. Harper, Radiation Damage studies of the silicon microstrip detectors and finding an intermediate mass Higgs Boson at ATLAS, Ph.D. Thesis, Sheffield University, U.K. (2001).

[59] A. Chilingarov, Temperature dependence of the current generated in Si bulk, 2013 JINST 8 P10003.

[60] I. Mandić, V. Cindro, A. Gorišek, G. Kramberger and M. Mikuž, Online integrating radiation monitoring system for the ATLAS detector at the Large Hadron Collider, IEEE Trans. Nucl. Sci. 54 (2007) 1143.

[61] I. Mandić et al., First Results from the Online Radiation Dose Monitoring System in ATLAS experiment, IEEE Nucl. Sci. Symp. Conf. Rec. 2011 (2011) 1107.

[62] A. Dafinca, J. Henderson and A.R. Weidberg on behalf of the ATLAS SCT collaboration, Single event upset studies using the ATLAS SCT, 2014 JINST 9 C01050.

[63] L. Eklund et al., SEU rate estimates for the ATLAS/SCT front-end ASIC, Nucl. Instrum. Meth. A 515 (2003) 415.

[64] J.D. Dowell et al., Single event upset studies with the optical links of the ATLAS semiconductor tracker, Nucl. Instrum. Meth. A 481 (2002) 575. 


\section{The ATLAS collaboration}

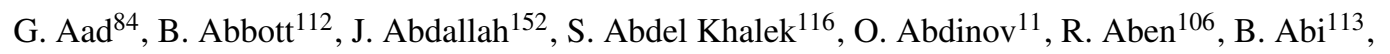
M. Abolins ${ }^{89}$, O.S. AbouZeid ${ }^{159}$, H. Abramowicz ${ }^{154}$, H. Abreu ${ }^{137}$, R. Abreu ${ }^{30}$, Y. Abulaiti ${ }^{147 a, 147 b}$, B.S. Acharya ${ }^{165 a, 165 b, a}$, L. Adamczyk ${ }^{38 a}$, D.L. Adams ${ }^{25}$, J. Adelman ${ }^{177}$, S. Adomeit ${ }^{99}$, T. Adye ${ }^{130}$, T. Agatonovic-Jovin ${ }^{13 b}$, J.A. Aguilar-Saavedra ${ }^{125 f, 125 a}$, M. Agustoni ${ }^{17}$, S.P. Ahlen ${ }^{22}$, A. Ahmad ${ }^{149}$, F. Ahmadov ${ }^{64, b}$, G. Aielli ${ }^{134 a, 134 b}$, T.P.A. Åkesson ${ }^{80}$, G. Akimoto ${ }^{156}$, A.V. Akimov ${ }^{95}$, G.L. Alberghi ${ }^{20 a, 20 b}$, J. Albert ${ }^{170}$, S. Albrand ${ }^{55}$, M.J. Alconada Verzini ${ }^{70}$, M. Aleksa ${ }^{30}$, I.N. Aleksandrov ${ }^{64}$, C. Alexa ${ }^{26 a}$, G. Alexander ${ }^{154}$, G. Alexandre ${ }^{49}$, T. Alexopoulos ${ }^{10}$, M. Alhroob ${ }^{165 a, 165 c}$, G. Alimonti ${ }^{90 a}$, L. Alio $^{84}$, J. Alison ${ }^{31}$, B.M.M. Allbrooke ${ }^{18}$, L.J. Allison ${ }^{71}$, P.P. Allport ${ }^{73}$, S.E. Allwood-Spiers ${ }^{53}$, J. Almond ${ }^{83}$, A. Aloisio ${ }^{103 a, 103 b}$, A. Alonso ${ }^{36}$, F. Alonso $^{70}$, C. Alpigiani ${ }^{75}$, A. Altheimer ${ }^{35}$, B. Alvarez Gonzalez ${ }^{89}$, M.G. Alviggi ${ }^{103 a, 103 b}$, K. Amako ${ }^{65}$, Y. Amaral Coutinho ${ }^{24 a}$, C. Amelung ${ }^{23}$, D. Amidei ${ }^{88}$,

S.P. Amor Dos Santos ${ }^{125 a, 125 c}$, A. Amorim ${ }^{125 a, 125 b}$, S. Amoroso ${ }^{48}$, N. Amram ${ }^{154}$, G. Amundsen ${ }^{23}$, C. Anastopoulos $^{140}$, L.S. Ancu ${ }^{49}$, N. Andari ${ }^{30}$, T. Andeen ${ }^{35}$, C.F. Anders ${ }^{58 b}$, G. Anders ${ }^{30}$, K.J. Anderson ${ }^{31}$, A. Andreazza ${ }^{90 a}, 90 b$, V. Andrei ${ }^{58 a}$, X.S. Anduaga ${ }^{70}$, S. Angelidakis ${ }^{9}$, I. Angelozzi ${ }^{106}$, P. Anger ${ }^{44}$, A. Angerami ${ }^{35}$, F. Anghinolfi ${ }^{30}$, A.V. Anisenkov ${ }^{108}$, N. Anjos ${ }^{125 a}$, A. Annovi ${ }^{47}$, A. Antonaki ${ }^{9}$, M. Antonelli ${ }^{47}$, A. Antonov ${ }^{97}$, J. Antos ${ }^{145 b}$, F. Anulli ${ }^{133 a}$, M. Aoki ${ }^{65}$, L. Aperio Bella ${ }^{18}$, R. Apolle ${ }^{119, c}$, G. Arabidze ${ }^{89}$, I. Aracena ${ }^{144}$, Y. Arai ${ }^{65}$, J.P. Araque ${ }^{125 a}$, A.T.H. Arce ${ }^{45}$, J-F. Arguin ${ }^{94}$, S. Argyropoulos ${ }^{42}$, M. Arik ${ }^{19 a}$, A.J. Armbruster ${ }^{30}$, O. Arnaez ${ }^{82}$, V. Arnal ${ }^{81}$, H. Arnold ${ }^{48}$, O. Arslan ${ }^{21}$, A. Artamonov ${ }^{96}$, G. Artoni ${ }^{23}$, S. Asai ${ }^{156}$, N. Asbah ${ }^{94}$, A. Ashkenazi ${ }^{154}$, S. Ask ${ }^{28}$, B. Åsman ${ }^{147 a, 147 b}$, L. Asquith 6 , K. Assamagan ${ }^{25}$, R. Astalos ${ }^{145 a}$, M. Atkinson ${ }^{166}$, N.B. Atlay ${ }^{142}$, B. Auerbach ${ }^{6}$, K. Augsten ${ }^{127}$, M. Aurousseau ${ }^{146 b}$, G. Avolio $^{30}$, G. Azuelos ${ }^{94, d}$, Y. Azuma ${ }^{156}$, M.A. Baak ${ }^{30}$, C. Bacci ${ }^{135 a, 135 b}$, H. Bachacou ${ }^{137}$, K. Bachas ${ }^{155}$, M. Backes ${ }^{30}$, M. Backhaus ${ }^{30}$, J. Backus Mayes ${ }^{144}$, E. Badescu ${ }^{26 a}$, P. Bagiacchi ${ }^{133 a, 133 b}$, P. Bagnaia ${ }^{133 a, 133 b}$, Y. Bai ${ }^{33 a}$, T. Bain ${ }^{35}$, J.T. Baines ${ }^{130}$, O.K. Baker ${ }^{177}$, S. Baker ${ }^{77}$, P. Balek ${ }^{128}$, F. Balli ${ }^{137}$, E. Banas ${ }^{39}$, Sw. Banerjee ${ }^{174}$, D. Banfi ${ }^{30}$, A. Bangert ${ }^{151}$, A.A.E. Bannoura ${ }^{176}$, V. Bansal ${ }^{170}$, H.S. Bansil ${ }^{18}$, L. Barak ${ }^{173}$, S.P. Baranov ${ }^{95}$, E.L. Barberio ${ }^{87}$, D. Barberis ${ }^{50 a, 50 b}$, M. Barbero ${ }^{84}$, T. Barillari ${ }^{100}$, M. Barisonzi ${ }^{176}$, T. Barklow ${ }^{144}$, N. Barlow ${ }^{28}$, B.M. Barnett ${ }^{130}$, R.M. Barnett ${ }^{15}$, Z. Barnovska ${ }^{5}$, A. Baroncelli1 ${ }^{135 a}$, G. Barone ${ }^{49}$, A.J. Barr ${ }^{119}$, F. Barreiro ${ }^{81}$,

J. Barreiro Guimarães da Costa ${ }^{57}$, R. Bartoldus ${ }^{144}$, A.E. Barton ${ }^{71}$, P. Bartos ${ }^{145 a}$, V. Bartsch ${ }^{150}$, A. Bassalat ${ }^{116}$, A. Basye ${ }^{166}$, R.L. Bates ${ }^{53}$, L. Batkova ${ }^{145 a}$, J.R. Batley ${ }^{28}$, M. Battistin ${ }^{30}$, F. Bauer ${ }^{137}$, H.S. Bawa ${ }^{144, e}$, T. Beau ${ }^{79}$, P.H. Beauchemin ${ }^{162}$, R. Beccherle ${ }^{123 a, 123 b}$, P. Bechtle $^{21}$, H.P. Beck ${ }^{17}$, K. Becker ${ }^{176}$, S. Becker ${ }^{99}$, M. Beckingham ${ }^{139}$, C. Becot ${ }^{116}$, A.J. Beddall ${ }^{19 c}$, A. Beddall ${ }^{19 c}$, S. Bedikian ${ }^{177}$, V.A. Bednyakov ${ }^{64}$, C.P. Bee ${ }^{149}$, L.J. Beemster ${ }^{106}$, T.A. Beermann ${ }^{176}$, M. Begel ${ }^{25}$, K. Behr ${ }^{119}$,

C. Belanger-Champagne ${ }^{86}$, P.J. Bell ${ }^{49}$, W.H. Bell ${ }^{49}$, G. Bella ${ }^{154}$, L. Bellagamba ${ }^{20 a}$, A. Bellerive ${ }^{29}$, M. Bellomo ${ }^{85}$, A. Belloni ${ }^{57}$, K. Belotskiy ${ }^{97}$, O. Beltramello ${ }^{30}$, O. Benary ${ }^{154}$, D. Benchekroun ${ }^{136 a}$, K. Bendtz ${ }^{147 a, 147 b}$, N. Benekos ${ }^{166}$, Y. Benhammou ${ }^{154}$, E. Benhar Noccioli ${ }^{49}$, J.A. Benitez Garcia ${ }^{160 b}$, D.P. Benjamin ${ }^{45}$, J.R. Bensinger ${ }^{23}$, K. Benslama ${ }^{131}$, S. Bentvelsen ${ }^{106}$, D. Berge ${ }^{106}$,

E. Bergeaas Kuutmann ${ }^{16}$, N. Berger ${ }^{5}$, F. Berghaus ${ }^{170}$, E. Berglund ${ }^{106}$, J. Beringer ${ }^{15}$, J. Bernabéu ${ }^{168}$, C. Bernard ${ }^{22}$, P. Bernat ${ }^{77}$, C. Bernius ${ }^{78}$, F.U. Bernlochner ${ }^{170}$, T. Berry ${ }^{76}$, P. Berta ${ }^{128}$, C. Bertella ${ }^{84}$, F. Bertolucci123a,123b, M.I. Besana ${ }^{90 a}$, G.J. Besjes ${ }^{105}$, O. Bessidskaia ${ }^{147 a, 147 b}$, N. Besson ${ }^{137}$, C. Betancourt ${ }^{48}$, S. Bethke ${ }^{100}$, W. Bhimji ${ }^{46}$, R.M. Bianchi ${ }^{124}$, L. Bianchini' ${ }^{23}$, M. Bianco ${ }^{30}$, O. Biebel ${ }^{99}$, S.P. Bieniek ${ }^{77}$, K. Bierwagen ${ }^{54}$, J. Biesiada ${ }^{15}$, M. Biglietti135a, J. Bilbao De Mendizabal ${ }^{49}$, H. Bilokon ${ }^{47}$, M. Bindi ${ }^{54}$, S. Binet ${ }^{116}$, A. Bingul ${ }^{19 c}$, C. Bini1 ${ }^{133 a, 133 b}$, C.W. Black ${ }^{151}$, J.E. Black ${ }^{144}$, K.M. Black ${ }^{22}$, D. Blackburn ${ }^{139}$, R.E. Blair ${ }^{6}$, J.-B. Blanchard ${ }^{137}$, T. Blazek ${ }^{145 a}$, I. Bloch ${ }^{42}$, C. Blocker ${ }^{23}$, W. Blum ${ }^{82, *}$, U. Blumenschein ${ }^{54}$, G.J. Bobbink ${ }^{106}$, V.S. Bobrovnikov ${ }^{108}$, S.S. Bocchetta ${ }^{80}$, A. Bocci ${ }^{45}$, C.R. Boddy ${ }^{119}$, M. Boehler ${ }^{48}$, J. Boek ${ }^{176}$, T.T. Boek ${ }^{176}$, J.A. Bogaerts ${ }^{30}$, A.G. Bogdanchikov ${ }^{108}$, A. Bogouch ${ }^{91, *}$, C. Bohm ${ }^{147 a}$, J. Bohm ${ }^{126}$, V. Boisvert ${ }^{76}$, T. Bold ${ }^{38 a}$, V. Boldea ${ }^{26 a}$, A.S. Boldyrev ${ }^{98}$, M. Bomben ${ }^{79}$, M. Bona ${ }^{75}$, M. Boonekamp ${ }^{137}$, A. Borisov ${ }^{129}$, G. Borissov ${ }^{71}$, M. Borri ${ }^{83}$, S. Borroni ${ }^{42}$, J. Bortfeldt ${ }^{99}$, 


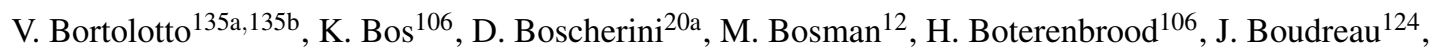
J. Bouffard ${ }^{2}$, E.V. Bouhova-Thacker ${ }^{71}$, D. Boumediene ${ }^{34}$, C. Bourdarios ${ }^{116}$, N. Bousson ${ }^{113}$, S. Boutouil ${ }^{136 d}$, A. Boveia ${ }^{31}$, J. Boyd ${ }^{30}$, I.R. Boyko ${ }^{64}$, I. Bozovic-Jelisavcic ${ }^{13 b}$, J. Bracinik ${ }^{18}$, P. Branchini ${ }^{135 a}$, A. Brandt ${ }^{8}$, G. Brandt ${ }^{15}$, O. Brandt ${ }^{58 a}$, U. Bratzler ${ }^{157}$, B. Brau ${ }^{85}$, J.E. Brau ${ }^{115}$, H.M. Braun ${ }^{176, *}$, S.F. Brazzale ${ }^{165 a, 165 c}$, B. Brelier ${ }^{159}$, K. Brendlinger ${ }^{121}$, A.J. Brennan ${ }^{87}$, R. Brenner ${ }^{167}$, S. Bressler ${ }^{173}$, K. Bristow ${ }^{146 c}$, T.M. Bristow ${ }^{46}$, D. Britton ${ }^{53}$, F.M. Brochu ${ }^{28}$, I. Brock ${ }^{21}$, R. Brock ${ }^{89}$, C. Bromberg ${ }^{89}$, J. Bronner ${ }^{100}$, G. Brooijmans ${ }^{35}$, T. Brooks ${ }^{76}$, W.K. Brooks ${ }^{32 b}$, J. Brosamer ${ }^{15}$, E. Brost ${ }^{115}$, G. Brown ${ }^{83}$, J. Brown ${ }^{55}$, P.A. Bruckman de Renstrom ${ }^{39}$, D. Bruncko ${ }^{145 b}$, R. Bruneliere ${ }^{48}$, S. Brunet $^{60}$, A. Bruni ${ }^{20 a}$, G. Bruni ${ }^{20 a}$, M. Bruschi ${ }^{20 a}$, L. Bryngemark $^{80}$, T. Buanes $^{14}$, Q. Buat ${ }^{143}$, F. Bucci $^{49}$, P. Buchholz ${ }^{142}$, R.M. Buckingham ${ }^{119}$, A.G. Buckley ${ }^{53}$, S.I. Buda ${ }^{26 a}$, I.A. Budagov ${ }^{64}$, F. Buehrer ${ }^{48}$, L. Bugge ${ }^{118}$, M.K. Bugge ${ }^{118}$, O. Bulekov ${ }^{97}$, A.C. Bundock ${ }^{73}$, H. Burckhart ${ }^{30}$, S. Burdin ${ }^{73}$, B. Burghgrave ${ }^{107}$, S. Burke ${ }^{130}$, I. Burmeister ${ }^{43}$, E. Busato ${ }^{34}$, D. Büscher ${ }^{48}$, V. Büscher ${ }^{82}$, P. Bussey ${ }^{53}$, C.P. Buszello ${ }^{167}$, B. Butler ${ }^{57}$, J.M. Butler ${ }^{22}$, A.I. Butt ${ }^{3}$, C.M. Buttar ${ }^{53}$, J.M. Butterworth ${ }^{77}$, P. Butti ${ }^{106}$, W. Buttinger ${ }^{28}$, A. Buzatu ${ }^{53}$, M. Byszewski ${ }^{10}$, S. Cabrera Urbán ${ }^{168}$, D. Caforio ${ }^{20 a, 20 b}$, O. Cakir ${ }^{4 a}$, P. Calafiura ${ }^{15}$, A. Calandri ${ }^{137}$, G. Calderini ${ }^{79}$, P. Calfayan ${ }^{99}$, R. Calkins ${ }^{107}$, L.P. Caloba ${ }^{24 a}$, D. Calvet ${ }^{34}$, S. Calvet ${ }^{34}$, R. Camacho Toro ${ }^{49}$, S. Camarda ${ }^{42}$, D. Cameron ${ }^{118}$, L.M. Caminada ${ }^{15}$,

R. Caminal Armadans ${ }^{12}$, S. Campana ${ }^{30}$, M. Campanelli ${ }^{77}$, A. Campoverde ${ }^{149}$, V. Canale ${ }^{103 a, 103 b}$, A. Canepa ${ }^{160 a}$, J. Cantero ${ }^{81}$, R. Cantrill ${ }^{76}$, T. Cao ${ }^{40}$, M.D.M. Capeans Garrido $^{30}$, I. Caprini $^{26 a}$, M. Caprini ${ }^{26 a}$, M. Capua ${ }^{37 a, 37 b}$, R. Caputo ${ }^{82}$, R. Cardarelli ${ }^{134 a}$, T. Carli $^{30}$, G. Carlino $^{103 a}$, L. Carminati90a,90b, S. Caron ${ }^{105}$, E. Carquin ${ }^{32 a}$, G.D. Carrillo-Montoya ${ }^{146 c}$, A.A. Carter ${ }^{75}$, J.R. Carter ${ }^{28}$, J. Carvalho ${ }^{125 a, 125 c}$, D. Casadei ${ }^{77}$, M.P. Casado ${ }^{12}$, E. Castaneda-Miranda ${ }^{146 b}$, A. Castelli ${ }^{106}$, V. Castillo Gimenez ${ }^{168}$, N.F. Castro ${ }^{125 a}$, P. Catastini ${ }^{57}$, A. Catinaccio ${ }^{30}$, J.R. Catmore ${ }^{118}$, A. Cattai ${ }^{30}$, G. Cattani ${ }^{134 a, 134 b}$, S. Caughron ${ }^{89}$, V. Cavaliere ${ }^{166}$, D. Cavalli ${ }^{90 a}$, M. Cavalli-Sforza ${ }^{12}$, V. Cavasinni ${ }^{123 a, 123 b}$, F. Ceradini ${ }^{135 a, 135 b}$, B. Cerio ${ }^{45}$, K. Cerny ${ }^{128}$, A.S. Cerqueira ${ }^{24 b}$, A. Cerri ${ }^{150}$, L. Cerrito ${ }^{75}$, F. Cerutti ${ }^{15}$, M. Cerv ${ }^{30}$, A. Cervelli ${ }^{17}$, S.A. Cetin ${ }^{19 b}$, A. Chafaq ${ }^{136 a}$, D. Chakraborty ${ }^{107}$, I. Chalupkova ${ }^{128}$, K. Chan ${ }^{3}$, P. Chang ${ }^{166}$, B. Chapleau ${ }^{86}$, J.D. Chapman ${ }^{28}$, D. Charfeddine ${ }^{116}$, D.G. Charlton ${ }^{18}$, C.C. Chau ${ }^{159}$, C.A. Chavez Barajas ${ }^{150}$, S. Cheatham ${ }^{86}$, A. Chegwidden ${ }^{89}$, S. Chekanov ${ }^{6}$, S.V. Chekulaev ${ }^{160 a}$, G.A. Chelkov ${ }^{64}$, M.A. Chelstowska ${ }^{88}$, C. Chen ${ }^{63}$, H. Chen ${ }^{25}$, K. Chen ${ }^{149}$, L. Chen ${ }^{33 d, f}$, S. Chen ${ }^{33 c}$, X. Chen ${ }^{146 c}$, Y. Chen ${ }^{35}$, H.C. Cheng ${ }^{88}$, Y. Cheng ${ }^{31}$, A. Cheplakov ${ }^{64}$, R. Cherkaoui El Moursli136e, V. Chernyatinn ${ }^{25, *}$, E. Cheu ${ }^{7}$, L. Chevalier ${ }^{137}$, V. Chiarella ${ }^{47}$, G. Chiefari ${ }^{103 a, 103 b}$, J.T. Childers ${ }^{6}$, A. Chilingarov ${ }^{71}$, G. Chiodini ${ }^{72 a}$, A.S. Chisholm ${ }^{18}$, R.T. Chislett $^{77}$, A. Chitan ${ }^{26 a}$, M.V. Chizhov ${ }^{64}$, S. Chouridou 9 , B.K.B. Chow ${ }^{99}$, I.A. Christidi ${ }^{77}$, D. Chromek-Burckhart ${ }^{30}$, M.L. Chu ${ }^{152}$, J. Chudoba ${ }^{126}$, J.J. Chwastowski ${ }^{39}$, L. Chytka ${ }^{114}$, G. Ciapetti ${ }^{133 a, 133 b}$, A.K. Ciftci ${ }^{4 a}$, R. Ciftci ${ }^{4 a}$, D. Cinca ${ }^{62}$, V. Cindro ${ }^{74}$, A. Ciocio ${ }^{15}$, P. Cirkovic ${ }^{13 b}$, Z.H. Citron ${ }^{173}$, M. Citterio ${ }^{90 a}$, M. Ciubancan ${ }^{26 a}$, A. Clark ${ }^{49}$, P.J. Clark ${ }^{46}$, R.N. Clarke ${ }^{15}$, W. Cleland ${ }^{124}$, J.C. Clemens ${ }^{84}$, C. Clement ${ }^{147 a, 147 b}$, Y. Coadou ${ }^{84}$, M. Cobal ${ }^{165 a, 165 c}$, A. Coccaro ${ }^{139}$, J. Cochran ${ }^{63}$, L. Coffey ${ }^{23}$, J.G. Cogan ${ }^{144}$, J. Coggeshall ${ }^{166}$, B. Cole ${ }^{35}$, S. Cole ${ }^{107}$, A.P. Colijn ${ }^{106}$, C. Collins-Tooth ${ }^{53}$, J. Collot ${ }^{55}$, T. Colombo ${ }^{58 c}$, G. Colon ${ }^{85}$, G. Compostella ${ }^{100}$, P. Conde Muiño ${ }^{125 a, 125 b}$, E. Coniavitis ${ }^{167}$, M.C. Conidi ${ }^{12}$, S.H. Connell ${ }^{146 b}$, I.A. Connelly ${ }^{76}$, S.M. Consonni ${ }^{90 a}, 90 b$, V. Consorti ${ }^{48}$, S. Constantinescu $^{26 a}$, C. Conta ${ }^{120 a, 120 b}$, G. Conti ${ }^{57}$, F. Conventi ${ }^{103 a, g}$, M. Cooke ${ }^{15}$, B.D. Cooper ${ }^{77}$, A.M. Cooper-Sarkar ${ }^{119}$, N.J. Cooper-Smith ${ }^{76}$, K. Copic ${ }^{15}$, T. Cornelissen ${ }^{176}$, M. Corradi ${ }^{20 a}$, F. Corriveau ${ }^{86, h}$, A. Corso-Radu ${ }^{164}$,

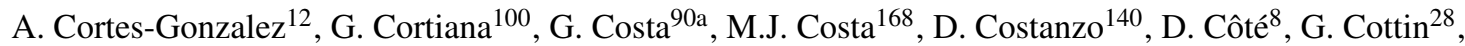
G. Cowan ${ }^{76}$, B.E. Cox $^{83}$, K. Cranmer ${ }^{109}$, G. Cree ${ }^{29}$, S. Crépé-Renaudin ${ }^{55}$, F. Crescioli ${ }^{79}$, M. Crispin Ortuzar ${ }^{119}$, M. Cristinziani ${ }^{21}$, V. Croft ${ }^{105}$, G. Crosetti ${ }^{37 a, 37 b}$, C.-M. Cuciuc ${ }^{26 a}$, C. Cuenca Almenar ${ }^{177}$, T. Cuhadar Donszelmann ${ }^{140}$, J. Cummings ${ }^{177}$, M. Curatolo ${ }^{47}$, C. Cuthbert ${ }^{151}$, H. Czirr ${ }^{142}$, P. Czodrowski ${ }^{3}$, Z. Czyczula ${ }^{177}$, S. D’Auria ${ }^{53}$, M. D’Onofrio ${ }^{73}$, M.J. Da Cunha Sargedas De Sousa ${ }^{125 a, 125 b}$, C. Da Via ${ }^{83}$, W. Dabrowski ${ }^{38 a}$, A. Dafinca ${ }^{119}$, T. Dai ${ }^{88}$,

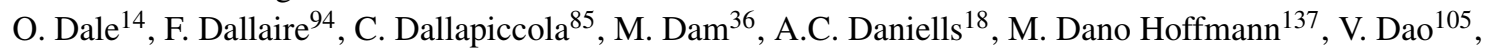




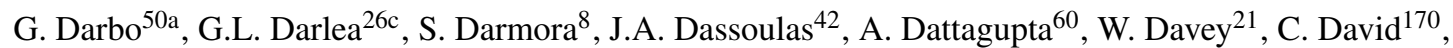
T. Davidek ${ }^{128}$, E. Davies ${ }^{119, c}$, M. Davies ${ }^{154}$, O. Davignon ${ }^{79}$, A.R. Davison ${ }^{77}$, P. Davison ${ }^{77}$, Y. Davygora ${ }^{58 a}$, E. Dawe ${ }^{143}$, I. Dawson ${ }^{140}$, R.K. Daya-Ishmukhametova ${ }^{23}$, K. De ${ }^{8}$, R. de Asmundis ${ }^{103 a}$, S. De Castro ${ }^{20 a, 20 b}$, S. De $\mathrm{Cecco}^{79}$, J. de Graat ${ }^{99}$, N. De Groot ${ }^{105}$, P. de Jong ${ }^{106}$, H. De la Torre ${ }^{81}$, F. De Lorenzi ${ }^{63}$, L. De Nooij ${ }^{106}$, D. De Pedis ${ }^{133 a}$, A. De Salvo ${ }^{133 a}$, U. De Sanctis ${ }^{165 a}{ }^{165 b}$, A. De Santo ${ }^{150}$, J.B. De Vivie De Regie ${ }^{116}$, G. De Zorzi ${ }^{133 a}, 133 b$, W.J. Dearnaley ${ }^{71}$, R. Debbe ${ }^{25}$, C. Debenedetti ${ }^{46}$, B. Dechenaux ${ }^{55}$, D.V. Dedovich ${ }^{64}$, J. Degenhardt ${ }^{121}$, I. Deigaard ${ }^{106}$, J. Del Peso ${ }^{81}$, T. Del Prete ${ }^{123 a, 123 b}$, F. Deliot ${ }^{137}$, C.M. Delitzsch ${ }^{49}$, M. Deliyergiyev ${ }^{74}$, A. Dell'Acqua ${ }^{30}$, L. Dell' Asta ${ }^{22}$, M. Dell'Orso ${ }^{123 a, 123 b}$, M. Della Pietra ${ }^{103 a, g}$, D. della Volpe ${ }^{49}$, M. Delmastro ${ }^{5}$, P.A. Delsart ${ }^{55}$, C. Deluca $^{106}$, S. Demers ${ }^{177}$, M. Demichev ${ }^{64}$, A. Demilly ${ }^{79}$, S.P. Denisov ${ }^{129}$, D. Derendarz ${ }^{39}$, J.E. Derkaoui ${ }^{136 d}$, F. Derue ${ }^{79}$, P. Dervan $^{73}$, K. Desch $^{21}$, C. Deterre ${ }^{42}$, P.O. Deviveiros ${ }^{106}$, A. Dewhurst ${ }^{130}$, S. Dhaliwal ${ }^{106}$, A. Di Ciaccio ${ }^{134 a, 134 b}$, L. Di Ciaccio 5 , A. Di Domenico ${ }^{133 a, 133 b}$, C. Di Donato ${ }^{103 a, 103 b}$, A. Di Girolamo ${ }^{30}$, B. Di Girolamo ${ }^{30}$, A. Di Mattia ${ }^{153}$, B. Di Micco ${ }^{135 a, 135 b}$, R. Di Nardo ${ }^{47}$, A. Di Simone ${ }^{48}$, R. Di Sipio ${ }^{20 a, 20 b}$, D. Di Valentino ${ }^{29}$, M.A. Diaz ${ }^{32 a}$, E.B. Diehl ${ }^{88}$, J. Dietrich ${ }^{42}$, T.A. Dietzsch ${ }^{58 a}$, S. Diglio ${ }^{84}$,

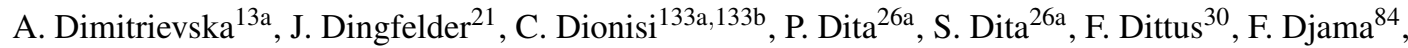
T. Djobava ${ }^{51 b}$, M.A.B. do Vale ${ }^{24 c}$, A. Do Valle Wemans ${ }^{125 a, 125 g}$, T.K.O. Doan ${ }^{5}$, D. Dobos ${ }^{30}$, E. Dobson ${ }^{77}$, C. Doglioni ${ }^{49}$, T. Doherty ${ }^{53}$, T. Dohmae ${ }^{156}$, J. Dolejsi $^{128}$, Z. Dolezal ${ }^{128}$, B.A. Dolgoshein ${ }^{97, *}$,

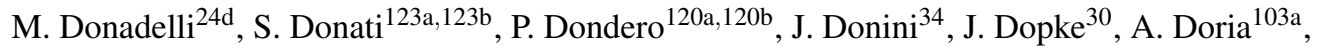
A. Dos Anjos ${ }^{174}$, M.T. Dova ${ }^{70}$, A.T. Doyle ${ }^{53}$, M. Dris ${ }^{10}$, J. Dubbert ${ }^{88}$, S. Dube $^{15}$, E. Dubreuil ${ }^{34}$, E. Duchovni ${ }^{173}$, G. Duckeck ${ }^{99}$, O.A. Ducu ${ }^{26 a}$, D. Duda ${ }^{176}$, A. Dudarev ${ }^{30}$, F. Dudziak ${ }^{63}$, L. Duflot ${ }^{116}$,

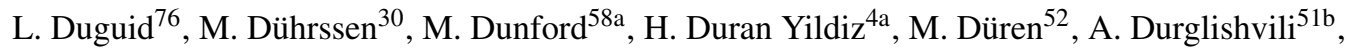
M. Dwuznik ${ }^{38 a}$, M. Dyndal ${ }^{38 a}$, J. Ebke ${ }^{99}$, W. Edson ${ }^{2}$, N.C. Edwards ${ }^{46}$, W. Ehrenfeld ${ }^{21}$, T. Eifert ${ }^{144}$, G. Eigen ${ }^{14}$, K. Einsweiler ${ }^{15}$, T. Ekelof ${ }^{167}$, M. El Kacimi ${ }^{136 c}$, M. Ellert ${ }^{167}$, S. Elles ${ }^{5}$, F. Ellinghaus ${ }^{82}$, N. Ellis ${ }^{30}$, J. Elmsheuser ${ }^{99}$, M. Elsing ${ }^{30}$, D. Emeliyanov ${ }^{130}$, Y. Enari ${ }^{156}$, O.C. Endner ${ }^{82}$, M. Endo ${ }^{117}$, R. Engelmann ${ }^{149}$, J. Erdmann ${ }^{177}$, A. Ereditato ${ }^{17}$, D. Eriksson ${ }^{147 a}$, G. Ernis ${ }^{176}$, J. Ernst ${ }^{2}$, M. Ernst ${ }^{25}$, J. Ernwein ${ }^{137}$, D. Errede ${ }^{166}$, S. Errede ${ }^{166}$, E. Ertel ${ }^{82}$, M. Escalier ${ }^{116}$, H. Esch $^{43}$, C. Escobar ${ }^{124}$, B. Esposito ${ }^{47}$, A.I. Etienvre ${ }^{137}$, E. Etzion ${ }^{154}$, H. Evans ${ }^{60}$, L. Fabbri ${ }^{20 a}{ }^{20 b}$, G. Facini ${ }^{30}$, R.M. Fakhrutdinov ${ }^{129}$, S. Falciano ${ }^{133 a}$, J. Faltova ${ }^{128}$, Y. Fang ${ }^{33 a}$, M. Fanti ${ }^{90 a, 90 b}$, A. Farbin ${ }^{8}$, A. Farilla ${ }^{135 a}$, T. Farooque ${ }^{12}$, S. Farrell ${ }^{164}$, S.M. Farrington ${ }^{171}$, P. Farthouat ${ }^{30}$, F. Fassi ${ }^{168}$, P. Fassnacht ${ }^{30}$, D. Fassouliotis ${ }^{9}$, A. Favareto ${ }^{50 a, 50 b}$, L. Fayard ${ }^{116}$, P. Federic ${ }^{145 a}$, O.L. Fedin ${ }^{122, i}$, W. Fedorko ${ }^{169}$, M. Fehling-Kaschek ${ }^{48}$, S. Feigl ${ }^{30}$, L. Feligioni ${ }^{84}$, C. Feng ${ }^{33 d}$, E.J. Feng ${ }^{6}$, H. Feng ${ }^{88}$, A.B. Fenyuk ${ }^{129}$, S. Fernandez Perez ${ }^{30}$, S. Ferrag ${ }^{53}$, J. Ferrando ${ }^{53}$, A. Ferrari ${ }^{167}$, P. Ferrari ${ }^{106}$, R. Ferrari ${ }^{120 a}$, D.E. Ferreira de Lima $^{53}$, A. Ferrer ${ }^{168}$, D. Ferrere ${ }^{49}$, C. Ferretti ${ }^{88}$, A. Ferretto Parodi ${ }^{50 a, 50 b}$, M. Fiascaris ${ }^{31}$, F. Fiedler ${ }^{82}$, A. Filipčič ${ }^{74}$, M. Filipuzzi ${ }^{42}$, F. Filthaut ${ }^{105}$, M. Fincke-Keeler ${ }^{170}$, K.D. Finelli ${ }^{151}$, M.C.N. Fiolhais ${ }^{125 a, 125 c}{ }^{\text {, L. Fiorini }}{ }^{168}$, A. Firan ${ }^{40}$, J. Fischer ${ }^{176}$, W.C. Fisher ${ }^{89}$, E.A. Fitzgerald ${ }^{23}$, M. Flechl ${ }^{48}$, I. Fleck ${ }^{142}$, P. Fleischmann ${ }^{175}$, S. Fleischmann ${ }^{176}$, G.T. Fletcher ${ }^{140}$, G. Fletcher ${ }^{75}$, T. Flick ${ }^{176}$, A. Floderus ${ }^{80}$, L.R. Flores Castillo ${ }^{174}$, A.C. Florez Bustos ${ }^{160 b}$, M.J. Flowerdew ${ }^{100}$, A. Formica ${ }^{137}$, A. Forti ${ }^{83}$, D. Fortin ${ }^{160 a}$, D. Fournier ${ }^{16}$, H. Fox ${ }^{71}$, S. Fracchia ${ }^{12}$, P. Francavilla ${ }^{79}$, M. Franchini ${ }^{20 a, 20 b}$, S. Franchino ${ }^{30}$, D. Francis ${ }^{30}$, M. Franklin ${ }^{57}$, S. Franz ${ }^{61}$, M. Fraternali ${ }^{120 a, 120 b}$, S.T. French ${ }^{28}$, C. Friedrich $^{42}$, F. Friedrich ${ }^{44}$, D. Froidevaux ${ }^{30}$, J.A. Frost ${ }^{28}$, C. Fukunaga ${ }^{157}$, E. Fullana Torregrosa ${ }^{82}$, B.G. Fulsom ${ }^{144}$, J. Fuster ${ }^{168}$, C. Gabaldon ${ }^{55}$, O. Gabizon ${ }^{173}$, A. Gabrielli20a,20b ${ }^{20}$ A. Gabrielli ${ }^{133 a, 133 b}$, S. Gadatsch ${ }^{106}$, S. Gadomski ${ }^{49}$, G. Gagliardi ${ }^{50 a, 50 b}$, P. Gagnon ${ }^{60}$, C. Galea ${ }^{105}$, B. Galhardo ${ }^{125 a, 125 c}$, E.J. Gallas ${ }^{119}$, V. Gallo ${ }^{17}$, B.J. Gallop ${ }^{130}$, P. Gallus ${ }^{127}$, G. Galster ${ }^{36}$, K.K. Gan ${ }^{110}$, R.P. Gandrajula ${ }^{62}$, J. Gao ${ }^{33 b, f}$, Y.S. Gao ${ }^{144, e}$, F.M. Garay Walls ${ }^{46}$, F. Garberson ${ }^{177}$, C. García ${ }^{168}$, C. Garcia Argos ${ }^{168}$, J.E. García Navarro ${ }^{168}$, M. Garcia-Sciveres ${ }^{15}$, R.W. Gardner ${ }^{31}$, N. Garelli ${ }^{144}$, V. Garonne ${ }^{30}$, C. Gatti ${ }^{47}$, G. Gaudio ${ }^{120 a}$, B. Gaur ${ }^{142}$, L. Gauthier ${ }^{94}$, P. Gauzzi ${ }^{133 a, 133 b}$, I.L. Gavrilenko ${ }^{95}$, C. Gay ${ }^{169}$, G. Gaycken ${ }^{21}$, E.N. Gazis ${ }^{10}$, P. Ge ${ }^{33 d}$, Z. Gecse ${ }^{169}$, C.N.P. Gee ${ }^{130}$, D.A.A. Geerts ${ }^{106}$, Ch. Geich-Gimbel ${ }^{21}$, K. Gellerstedt ${ }^{147 a, 147 b}$, C. Gemme ${ }^{50 a}$,

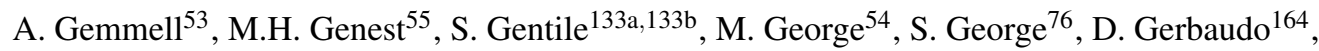


A. Gershon ${ }^{154}$, H. Ghazlane ${ }^{136 b}$, N. Ghodbane ${ }^{34}$, B. Giacobbe ${ }^{20 a}$, S. Giagu ${ }^{133 a, 133 b}$, V. Giangiobbe ${ }^{12}$, P. Giannetti ${ }^{123 a, 123 b}$, F. Gianotti ${ }^{30}$, B. Gibbard ${ }^{25}$, S.M. Gibson ${ }^{76}$, M. Gilchriese ${ }^{15}$, T.P.S. Gillam ${ }^{28}$, D. Gillberg ${ }^{30}$, G. Gilles ${ }^{34}$, D.M. Gingrich ${ }^{3, d}$, N. Giokaris ${ }^{9}$, M.P. Giordani ${ }^{165 a, 165 c}$, R. Giordano ${ }^{103 a, 103 b}$, F.M. Giorgi ${ }^{16}$, P.F. Giraud ${ }^{137}$, D. Giugni ${ }^{90 a}$, C. Giuliani ${ }^{48}$, M. Giulini ${ }^{58 b}$, B.K. Gjelsten ${ }^{118}$, I. Gkialas ${ }^{155, j}$, L.K. Gladilin ${ }^{98}$, C. Glasman ${ }^{81}$, J. Glatzer ${ }^{30}$, P.C.F. Glaysher ${ }^{46}$, A. Glazov ${ }^{42}$, G.L. Glonti ${ }^{64}$, M. Goblirsch-Kolb ${ }^{100}$, J.R. Goddard ${ }^{75}$, J. Godfrey ${ }^{143}$, J. Godlewski ${ }^{30}$, C. Goeringer ${ }^{82}$, S. Goldfarb ${ }^{88}$, T. Golling ${ }^{177}$, D. Golubkov ${ }^{129}$, A. Gomes ${ }^{125 a, 125 b, 125 d}$, L.S. Gomez Fajardo ${ }^{42}$, R. Gonçalo ${ }^{125 a}$, J. Goncalves Pinto Firmino Da Costa ${ }^{42}$, L. Gonella ${ }^{21}$, S. González de la Hoz ${ }^{168}$, G. Gonzalez Parra ${ }^{12}$, M.L. Gonzalez Silva ${ }^{27}$, S. Gonzalez-Sevilla ${ }^{49}$, M.J. Goodrick ${ }^{28}$, L. Goossens ${ }^{30}$, P.A. Gorbounov ${ }^{96}$, H.A. Gordon ${ }^{25}$, I. Gorelov ${ }^{104}$, G. Gorfine ${ }^{176}$, B. Gorini ${ }^{30}$, E. Gorini ${ }^{72 a, 72 b}$, A. Gorišek ${ }^{74}$, E. Gornicki ${ }^{39}$, A.T. Goshaw ${ }^{6}$, C. Gössling ${ }^{43}$, M.I. Gostkin ${ }^{64}$, M. Gouighri ${ }^{136 a}$, D. Goujdami ${ }^{136 c}$, M.P. Goulette ${ }^{49}$, A.G. Goussiou ${ }^{139}$, C. Goy ${ }^{5}$, S. Gozpinar ${ }^{23}$, H.M.X. Grabas ${ }^{137}$, L. Graber ${ }^{54}$, I. Grabowska-Bold ${ }^{38 a}$, P. Grafström ${ }^{20 a, 20 b}$, K-J. Grahn ${ }^{42}$, J. Gramling ${ }^{49}$, E. Gramstad ${ }^{118}$, S. Grancagnolo ${ }^{16}$, V. Grassi ${ }^{149}$, V. Gratchev ${ }^{122}$, H.M. Gray ${ }^{30}$, E. Graziani ${ }^{135 a}$, O.G. Grebenyuk ${ }^{122}$, Z.D. Greenwood ${ }^{78, k}$, K. Gregersen ${ }^{77}$, I.M. Gregor ${ }^{42}$, P. Grenier ${ }^{144}$, J. Griffiths ${ }^{8}$, N. Grigalashvili ${ }^{64}$, A.A. Grillo ${ }^{138}$, K. Grimm ${ }^{71}$, S. Grinstein ${ }^{12, l}$, Ph. Gris ${ }^{34}$, Y.V. Grishkevich ${ }^{98}$, J.-F. Grivaz ${ }^{116}$, J.P. Grohs ${ }^{44}$, A. Grohsjean ${ }^{42}$, E. Gross ${ }^{173}$,

J. Grosse-Knetter ${ }^{54}$, G.C. Grossi ${ }^{134 a, 134 b}$, J. Groth-Jensen ${ }^{173}$, Z.J. Grout ${ }^{150}$, K. Grybel ${ }^{142}$, L. Guan ${ }^{33 b}$, F. Guescini ${ }^{49}$, D. Guest ${ }^{177}$, O. Gueta ${ }^{154}$, C. Guicheney ${ }^{34}$, E. Guido ${ }^{50 a, 50 b}$, T. Guillemin ${ }^{116}$, S. Guindon ${ }^{2}$, U. Gul $^{53}$, C. Gumpert ${ }^{44}$, J. Gunther ${ }^{127}$, J. Guo ${ }^{35}$, S. Gupta ${ }^{119}$, P. Gutierrez ${ }^{112}$, N.G. Gutierrez Ortiz ${ }^{53}$, C. Gutschow ${ }^{77}$, N. Guttman ${ }^{154}$, C. Guyot ${ }^{137}$, C. Gwenlan ${ }^{119}$, C.B. Gwilliam ${ }^{73}$, A. Haas ${ }^{109}$, C. Haber ${ }^{15}$, H.K. Hadavand ${ }^{8}$, N. Haddad ${ }^{136 e}$, P. Haefner ${ }^{21}$, S. Hageboeck ${ }^{21}$, Z. Hajduk ${ }^{39}$, H. Hakobyan ${ }^{178}$, M. Haleem ${ }^{42}$, D. Hall ${ }^{119}$, G. Halladjian ${ }^{89}$, K. Hamacher ${ }^{176}$, P. Hamal ${ }^{14}$, K. Hamano ${ }^{87}$, M. Hamer ${ }^{54}$, A. Hamilton ${ }^{146 a}$, S. Hamilton ${ }^{162}$, P.G. Hamnett ${ }^{42}$, L. Han ${ }^{33 b}$, K. Hanagaki ${ }^{117}$, K. Hanawa ${ }^{156}$, M. Hance ${ }^{15}$,

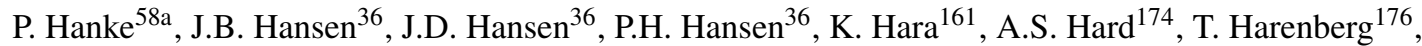
S. Harkusha ${ }^{91}$, D. Harper ${ }^{88}$, R.D. Harrington ${ }^{46}$, O.M. Harris ${ }^{139}$, P.F. Harrison ${ }^{171}$, F. Hartjes ${ }^{106}$, S. Hasegawa ${ }^{102}$, Y. Hasegawa ${ }^{141}$, A Hasib ${ }^{112}$, S. Hassani ${ }^{137}$, S. Haug ${ }^{17}$, M. Hauschild ${ }^{30}$, R. Hauser ${ }^{89}$, M. Havranek ${ }^{126}$, C.M. Hawkes ${ }^{18}$, R.J. Hawkings ${ }^{30}$, A.D. Hawkins ${ }^{80}$, T. Hayashi ${ }^{161}$, D. Hayden ${ }^{89}$, C.P. Hays ${ }^{119}$, H.S. Hayward ${ }^{73}$, S.J. Haywood ${ }^{130}$, S.J. Head ${ }^{18}$, T. Heck ${ }^{82}$, V. Hedberg ${ }^{80}$, L. Heelan ${ }^{8}$, S. Heim ${ }^{121}$, T. Heim ${ }^{176}$, B. Heinemann ${ }^{15}$, L. Heinrich ${ }^{109}$, S. Heisterkamp ${ }^{36}$, J. Hejbal ${ }^{126}$, L. Helary ${ }^{22}$, C. Heller ${ }^{99}$, M. Heller ${ }^{30}$, S. Hellman ${ }^{147 a, 147 b}$, D. Hellmich ${ }^{21}$, C. Helsens ${ }^{30}$, J. Henderson ${ }^{119}$, R.C.W. Henderson ${ }^{71}$, C. Hengler ${ }^{42}$, A. Henrichs ${ }^{177}$, A.M. Henriques Correia ${ }^{30}$, S. Henrot-Versille ${ }^{116}$, C. Hensel ${ }^{54}$, G.H. Herbert ${ }^{16}$, Y. Hernández Jiménez ${ }^{168}$, R. Herrberg-Schubert ${ }^{16}$, G. Herten ${ }^{48}$, R. Hertenberger ${ }^{99}$, L. Hervas ${ }^{30}$, G.G. Hesketh ${ }^{77}$, N.P. Hessey ${ }^{106}$, R. Hickling ${ }^{75}$, E. Higón-Rodriguez ${ }^{168}$, E. Hill ${ }^{170}$, J.C. Hill ${ }^{28}$, K.H. Hiller ${ }^{42}$, S. Hillert ${ }^{21}$, S.J. Hillier ${ }^{18}$, I. Hinchliffe ${ }^{15}$, E. Hines ${ }^{121}$, M. Hirose ${ }^{117}$, D. Hirschbuehl ${ }^{176}$, J. Hobbs ${ }^{149}$, N. Hod ${ }^{106}$, M.C. Hodgkinson ${ }^{140}$, P. Hodgson ${ }^{140}$, A. Hoecker ${ }^{30}$, M.R. Hoeferkamp ${ }^{104}$, J. Hoffman ${ }^{40}$, D. Hoffmann ${ }^{84}$, J.I. Hofmann ${ }^{58 a}$, M. Hohlfeld ${ }^{82}$, T.R. Holmes ${ }^{15}$, T.M. Hong ${ }^{121}$, L. Hooft van Huysduynen ${ }^{109}$, J-Y. Hostachy ${ }^{55}$, S. Hou ${ }^{152}$, A. Hoummada ${ }^{136 a}$, J. Howard ${ }^{119}$,

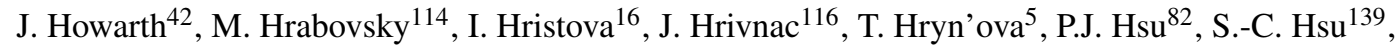

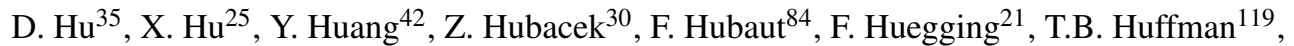
E.W. Hughes ${ }^{35}$, G. Hughes ${ }^{71}$, M. Huhtinen ${ }^{30}$, T.A. Hülsing ${ }^{82}$, M. Hurwitz ${ }^{15}$, N. Huseynov ${ }^{64, b}$, J. Huston ${ }^{89}$, J. Huth $^{57}$, G. Iacobucci ${ }^{49}$, G. Iakovidis ${ }^{10}$, I. Ibragimov ${ }^{142}$, L. Iconomidou-Fayard ${ }^{116}$, J. Idarraga ${ }^{116}$, E. Ideal ${ }^{177}$, P. Iengo ${ }^{103 a}$, O. Igonkina ${ }^{106}$, T. Iizawa ${ }^{172}$, Y. Ikegami ${ }^{65}$, K. Ikematsu ${ }^{142}$, M. Ikeno ${ }^{65}$,

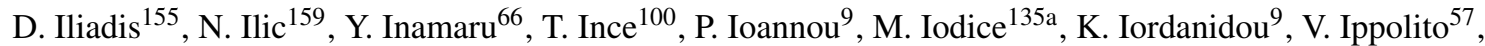
A. Irles Quiles ${ }^{168}$, C. Isaksson ${ }^{167}$, M. Ishino ${ }^{67}$, M. Ishitsuka ${ }^{158}$, R. Ishmukhametov ${ }^{110}$, C. Issever ${ }^{119}$, S. Istin ${ }^{19 a}$, J.M. Iturbe Ponce ${ }^{83}$, J. Ivarsson ${ }^{80}$, A.V. Ivashin ${ }^{129}$, W. Iwanski ${ }^{39}$, H. Iwasaki ${ }^{65}$, J.M. Izen ${ }^{41}$, V. Izzo ${ }^{103 a}$, B. Jackson ${ }^{121}$, J.N. Jackson ${ }^{73}$, M. Jackson ${ }^{73}$, P. Jackson ${ }^{1}$, M.R. Jaekel ${ }^{30}$, V. Jain ${ }^{2}$, K. Jakobs ${ }^{48}$, S. Jakobsen ${ }^{30}$, T. Jakoubek ${ }^{126}$, J. Jakubek ${ }^{127}$, D.O. Jamin ${ }^{152}$, D.K. Jana ${ }^{78}$, E. Jansen ${ }^{77}$, H. Jansen ${ }^{30}$, J. Janssen $^{21}$, M. Janus ${ }^{171}$, G. Jarlskog ${ }^{80}$, N. Javadov ${ }^{64, b}$, T. Javůrek ${ }^{48}$, L. Jeanty ${ }^{15}$, G.-Y. Jeng ${ }^{151}$, 
D. Jennens ${ }^{87}$, P. Jenni ${ }^{48, m}$, J. Jentzsch ${ }^{43}$, C. Jeske ${ }^{171}$, S. Jézéquel ${ }^{5}$, H. Ji $^{174}$, W. Ji ${ }^{82}$, J. Jia ${ }^{149}$, Y. Jiang ${ }^{33 b}$, M. Jimenez Belenguer ${ }^{42}$, S. Jin ${ }^{33 a}$, A. Jinaru ${ }^{26 a}$, O. Jinnouchi ${ }^{158}$, M.D. Joergensen ${ }^{36}$, K.E. Johansson ${ }^{147 a}$, P. Johansson ${ }^{140}$, K.A. Johns ${ }^{7}$, K. Jon-And ${ }^{147 a, 147 b}$, G. Jones ${ }^{171}$, R.W.L. Jones ${ }^{71}$, T.J. Jones ${ }^{73}$,

J. Jongmanns $^{58 a}$, P.M. Jorge ${ }^{125 a, 125 b}$, J. Joseph ${ }^{15}$, K.D. Joshi ${ }^{83}$, J. Jovicevic ${ }^{148}$, X. Ju ${ }^{174}$, C.A. Jung ${ }^{43}$, R.M. Jungst ${ }^{30}$, P. Jussel ${ }^{61}$, A. Juste Rozas ${ }^{12, l}$, M. Kaci ${ }^{168}$, A. Kaczmarska ${ }^{39}$, M. Kado ${ }^{116}$, H. Kagan ${ }^{110}$, M. Kagan ${ }^{144}$, E. Kajomovitz ${ }^{45}$, S. Kama ${ }^{40}$, N. Kanaya ${ }^{156}$, M. Kaneda ${ }^{30}$, S. Kaneti ${ }^{28}$, T. Kanno ${ }^{158}$, V.A. Kantserov ${ }^{97}$, J. Kanzaki ${ }^{65}$, B. Kaplan ${ }^{109}$, A. Kapliy ${ }^{31}$, D. Kar ${ }^{53}$, K. Karakostas ${ }^{10}$, N. Karastathis ${ }^{10}$, M. Karnevskiy ${ }^{82}$, S.N. Karpov ${ }^{64}$, K. Karthik ${ }^{109}$, V. Kartvelishvili ${ }^{71}$, A.N. Karyukhin ${ }^{129}$, L. Kashif ${ }^{174}$, G. Kasieczka ${ }^{58 b}$, R.D. Kass ${ }^{110}$, A. Kastanas ${ }^{14}$, Y. Kataoka ${ }^{156}$, A. Katre ${ }^{49}$, J. Katzy ${ }^{42}$, V. Kaushik ${ }^{7}$, K. Kawagoe ${ }^{69}$, T. Kawamoto ${ }^{156}$, G. Kawamura ${ }^{54}$, S. Kazama ${ }^{156}$, V.F. Kazanin ${ }^{108}$, M.Y. Kazarinov ${ }^{64}$, R. Keeler ${ }^{170}$, P.T. Keener ${ }^{121}$, R. Kehoe ${ }^{40}$, M. Keil ${ }^{54}$, J.S. Keller ${ }^{42}$, H. Keoshkerian ${ }^{5}$, O. Kepka ${ }^{126}$, B.P. Kerševan ${ }^{74}$, S. Kersten ${ }^{176}$, K. Kessoku ${ }^{156}$, J. Keung ${ }^{159}$, F. Khalil-zada ${ }^{11}$, H. Khandanyan ${ }^{147 a, 147 b}$, A. Khanov ${ }^{113}$, A. Khodinov ${ }^{97}$, A. Khomich ${ }^{58 a}$, T.J. Khoo ${ }^{28}$, G. Khoriauli ${ }^{21}$, A. Khoroshilov ${ }^{176}$, V. Khovanskiy ${ }^{96}$, E. Khramov ${ }^{64}$, J. Khubua ${ }^{51 b}$, H.Y. Kim ${ }^{8}$, H. Kim ${ }^{147 a, 147 b}$, S.H. Kim ${ }^{161}$, N. Kimura ${ }^{172}$, O. Kind ${ }^{16}$, B.T. King ${ }^{73}$, M. King ${ }^{168}$, R.S.B. King ${ }^{19}$, S.B. King ${ }^{169}$, J. Kirk ${ }^{130}$, A.E. Kiryunin ${ }^{100}$, T. Kishimoto ${ }^{66}$, D. Kisielewska ${ }^{38 a}$, F. Kiss ${ }^{48}$, T. Kitamura ${ }^{66}$, T. Kittelmann ${ }^{124}$, K. Kiuchi ${ }^{161}$, E. Kladiva ${ }^{145 b}$, M. Klein ${ }^{73}$, U. Klein ${ }^{73}$, K. Kleinknecht ${ }^{82}$, P. Klimek ${ }^{147 a, 147 b}$, A. Klimentov ${ }^{25}$, R. Klingenberg ${ }^{43}$, J.A. Klinger ${ }^{83}$, T. Klioutchnikova ${ }^{30}$, P.F. Klok ${ }^{105}$, E.-E. Kluge ${ }^{58 a}$, P. Kluit ${ }^{106}$, S. Kluth ${ }^{100}$, E. Kneringer ${ }^{61}$, E.B.F.G. Knoops ${ }^{84}$, A. Knue ${ }^{53}$, T. Kobayashi ${ }^{156}$, M. Kobel ${ }^{44}$, M. Kocian ${ }^{144}$, P. Kodys ${ }^{128}$, P. Koevesarki ${ }^{21}$, T. Koffas ${ }^{29}$, E. Koffeman ${ }^{106}$, L.A. Kogan ${ }^{119}$, S. Kohlmann ${ }^{176}$, Z. Kohout ${ }^{127}$, T. Kohriki ${ }^{65}$, T. Koi ${ }^{144}$, H. Kolanoski ${ }^{16}$, I. Koletsou ${ }^{5}$, J. Koll ${ }^{89}$, A.A. Komar ${ }^{95, *}$, Y. Komori ${ }^{156}$, T. Kondo ${ }^{65}$, N. Kondrashova ${ }^{42}$, K. Köneke ${ }^{48}$, A.C. König ${ }^{105}$, S. König ${ }^{82}$, T. Kono ${ }^{65, n}$, R. Konoplich ${ }^{109, o}$, N. Konstantinidis ${ }^{77}$, R. Kopeliansky ${ }^{153}$, S. Koperny ${ }^{38 a}$, L. Köpke ${ }^{82}$, A.K. Kopp ${ }^{48}$, K. Korcyl ${ }^{39}$, K. Kordas ${ }^{155}$, A. Korn ${ }^{77}$, A.A. Korol ${ }^{108, p}$, I. Korolkov ${ }^{12}$, E.V. Korolkova ${ }^{140}$, V.A. Korotkov ${ }^{129}$, O. Kortner ${ }^{100}$, S. Kortner ${ }^{100}$, V.V. Kostyukhin ${ }^{21}$, S. Kotov ${ }^{100}$, V.M. Kotov ${ }^{64}$, A. Kotwal ${ }^{45}$, C. Kourkoumelis 9 , V. Kouskoura ${ }^{155}$, A. Koutsman ${ }^{160 a}$, R. Kowalewski ${ }^{170}$, T.Z. Kowalski ${ }^{38 a}$, W. Kozanecki ${ }^{137}$, A.S. Kozhin ${ }^{129}$, V. Kral ${ }^{127}$, V.A. Kramarenko ${ }^{98}$, G. Kramberger ${ }^{74}$, D. Krasnopevtsev ${ }^{97}$, M.W. Krasny ${ }^{79}$, A. Krasznahorkay ${ }^{30}$, J.K. Kraus ${ }^{21}$, A. Kravchenko ${ }^{25}$, S. Kreiss ${ }^{109}$, M. Kretz ${ }^{58 c}$, J. Kretzschmar ${ }^{73}$, K. Kreutzfeldt ${ }^{52}$, P. Krieger ${ }^{159}$, K. Kroeninger ${ }^{54}$, H. Kroha ${ }^{100}$, J. Kroll ${ }^{121}$, J. Kroseberg ${ }^{21}$, J. Krstic ${ }^{13 a}$, U. Kruchonak ${ }^{64}$, H. Krüger ${ }^{21}$, T. Kruker ${ }^{17}$, N. Krumnack ${ }^{63}$, Z.V. Krumshteyn ${ }^{64}$, A. Kruse ${ }^{174}$, M.C. Kruse ${ }^{45}$, M. Kruskal ${ }^{22}$, P. Kubik ${ }^{128}$, T. Kubota ${ }^{87}$, S. Kuday ${ }^{4 a}$, S. Kuehn ${ }^{48}$, A. Kugel ${ }^{58 c}$, A. Kuhl ${ }^{138}$, T. Kuhl ${ }^{42}$, V. Kukhtin ${ }^{64}$, Y. Kulchitsky ${ }^{91}$, S. Kuleshov ${ }^{32 b}$, M. Kuna ${ }^{133 a, 133 b}$, J. Kunkle ${ }^{121}$, A. Kupco ${ }^{126}$, H. Kurashige ${ }^{66}$, Y.A. Kurochkin ${ }^{91}$, R. Kurumida ${ }^{66}$, V. Kus ${ }^{126}$, E.S. Kuwertz ${ }^{148}$, M. Kuze ${ }^{158}$, J. Kvita ${ }^{114}$, A. La Rosa ${ }^{49}$, L. La Rotonda ${ }^{37 a, 37 b}$, C. Lacasta ${ }^{168}$, F. Lacava133a,133b ${ }^{\text {, J. Lacey }}{ }^{29}$, H. Lacker ${ }^{16}$, D. Lacour ${ }^{79}$, V.R. Lacuesta ${ }^{168}$, E. Ladygin ${ }^{64}$, R. Lafaye ${ }^{5}$, B. Laforge ${ }^{79}$, T. Lagouri ${ }^{177}$, S. Lai ${ }^{48}$, H. Laier ${ }^{58 a}$, L. Lambourne ${ }^{77}$, S. Lammers ${ }^{60}$, C.L. Lampen ${ }^{7}$, W. Lampl ${ }^{7}$, E. Lançon ${ }^{137}$, U. Landgraf ${ }^{48}$, M.P.J. Landon ${ }^{75}$,

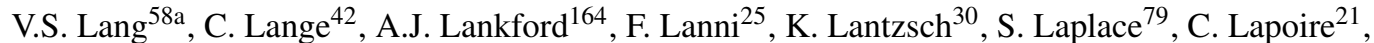

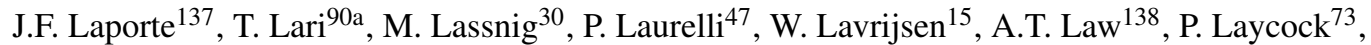
B.T. Le ${ }^{55}$, O. Le Dortz ${ }^{79}$, E. Le Guirriec ${ }^{84}$, E. Le Menedeu ${ }^{12}$, T. LeCompte ${ }^{6}$, F. Ledroit-Guillon ${ }^{55}$, C.A. Lee ${ }^{152}$, H. Lee ${ }^{106}$, J.S.H. Lee ${ }^{117}$, S.C. Lee ${ }^{152}$, L. Lee ${ }^{177}$, G. Lefebvre ${ }^{79}$, M. Lefebvre ${ }^{170}$, F. Legger ${ }^{99}$, C. Leggett ${ }^{15}$, A. Lehan ${ }^{73}$, M. Lehmacher ${ }^{21}$, G. Lehmann Miotto ${ }^{30}$, X. Lei ${ }^{7}$, A.G. Leister ${ }^{177}$, M.A.L. Leite ${ }^{24 d}$, R. Leitner ${ }^{128}$, D. Lellouch ${ }^{173}$, B. Lemmer ${ }^{54}$, K.J.C. Leney ${ }^{77}$, T. Lenz ${ }^{106}$, G. Lenzen ${ }^{176}$, B. Lenzi ${ }^{30}$, R. Leone ${ }^{7}$, K. Leonhardt ${ }^{44}$, S. Leontsinis ${ }^{10}$, C. Leroy ${ }^{94}$, C.G. Lester ${ }^{28}$, C.M. Lester ${ }^{121}$, M. Levchenko ${ }^{122}$, J. Levêque ${ }^{5}$, D. Levin ${ }^{88}$, L.J. Levinson ${ }^{173}$, M. Levy ${ }^{18}$, A. Lewis ${ }^{119}$, G.H. Lewis ${ }^{109}$,

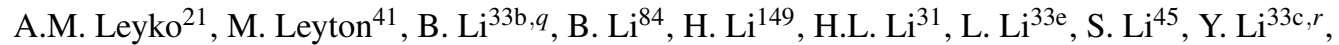
Z. Liang ${ }^{119, s}$, H. Liao ${ }^{34}$, B. Liberti ${ }^{134 a}$, P. Lichard ${ }^{30}$, K. Lie ${ }^{166}$, J. Liebal ${ }^{21}$, W. Liebig ${ }^{14}$, C. Limbach ${ }^{21}$, A. Limosani ${ }^{87}$, M. Limper ${ }^{62}$, S.C. Lin ${ }^{152, t}$, F. Linde ${ }^{106}$, B.E. Lindquist ${ }^{149}$, J.T. Linnemann ${ }^{89}$, E. Lipeles ${ }^{121}$,

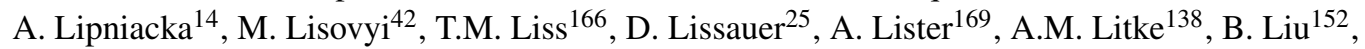




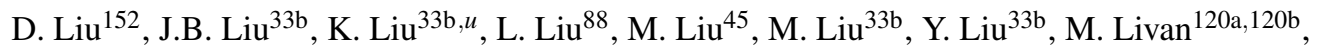
S.S.A. Livermore ${ }^{119}$, A. Lleres ${ }^{55}$, J. Llorente Merino ${ }^{81}$, S.L. Lloyd ${ }^{75}$, F. Lo Sterzo ${ }^{152}$, E. Lobodzinska ${ }^{42}$, P. Loch ${ }^{7}$, W.S. Lockman ${ }^{138}$, T. Loddenkoetter ${ }^{21}$, F.K. Loebinger ${ }^{83}$, A.E. Loevschall-Jensen ${ }^{36}$, A. Loginov $^{177}$, C.W. Loh ${ }^{169}$, T. Lohse ${ }^{16}$, K. Lohwasser ${ }^{48}$, M. Lokajicek ${ }^{126}$, V.P. Lombardo ${ }^{5}$, B.A. Long ${ }^{22}$, J.D. Long ${ }^{88}$, R.E. Long ${ }^{71}$, L. Lopes ${ }^{125 a}$, D. Lopez Mateos ${ }^{57}$, B. Lopez Paredes ${ }^{140}$, J. Lorenz ${ }^{99}$,

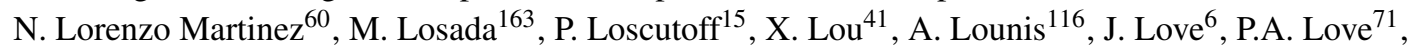
A.J. Lowe ${ }^{144, e}$, F. Lu ${ }^{33 a}$, H.J. Lubatti ${ }^{139}$, C. Luci ${ }^{133 a, 133 b}$, A. Lucotte ${ }^{55}$, F. Luehring ${ }^{60}$, W. Lukas ${ }^{61}$, L. Luminari ${ }^{133 a}$, O. Lundberg ${ }^{147 a, 147 b}$, B. Lund-Jensen ${ }^{148}$, M. Lungwitz ${ }^{82}$, D. Lynn ${ }^{25}$, R. Lysak ${ }^{126}$, E. Lytken ${ }^{80}$, H. Ma ${ }^{25}$, L.L. Ma ${ }^{33 d}$, G. Maccarrone ${ }^{47}$, A. Macchiolo ${ }^{100}$, J. Machado Miguens ${ }^{125 a, 125 b}$, D. Macina $^{30}$, D. Madaffari ${ }^{84}$, R. Madar $^{48}$, H.J. Maddocks ${ }^{71}$, W.F. Mader ${ }^{44}$, A. Madsen ${ }^{167}$, M. Maeno ${ }^{8}$, T. Maeno ${ }^{25}$, E. Magradze ${ }^{54}$, K. Mahboubi ${ }^{48}$, J. Mahlstedt ${ }^{106}$, S. Mahmoud ${ }^{73}$, C. Maiani ${ }^{137}$, C. Maidantchik ${ }^{24 a}$, A. Maio ${ }^{125 a, 125 b, 125 d}$, S. Majewski ${ }^{115}$, Y. Makida ${ }^{65}$, N. Makovec ${ }^{116}$, P. Mal ${ }^{137, v}$, B. Malaescu ${ }^{79}$, Pa. Malecki ${ }^{39}$, V.P. Maleev ${ }^{122}$, F. Malek ${ }^{55}$, U. Mallik ${ }^{62}$, D. Malon ${ }^{6}$, C. Malone ${ }^{144}$, S. Maltezos ${ }^{10}$, V.M. Malyshev ${ }^{108}$, S. Malyukov ${ }^{30}$, J. Mamuzic ${ }^{13 b}$, B. Mandelli ${ }^{30}$, L. Mandelli ${ }^{90 a}$, I. Mandić ${ }^{74}$, R. Mandrysch ${ }^{62}$, J. Maneira ${ }^{125 a, 125 b}$, A. Manfredini ${ }^{100}$, L. Manhaes de Andrade Filho ${ }^{24 b}$, J.A. Manjarres Ramos ${ }^{160 b}$, A. Mann ${ }^{99}$, P.M. Manning ${ }^{138}$, A. Manousakis-Katsikakis ${ }^{9}$, B. Mansoulie ${ }^{137}$, R. Mantifel ${ }^{86}$, L. Mapelli ${ }^{30}$, L. March ${ }^{168}$, J.F. Marchand ${ }^{29}$, G. Marchiori ${ }^{79}$, M. Marcisovsky ${ }^{126}$, C.P. Marino ${ }^{170}$, C.N. Marques ${ }^{125 a}$, F. Marroquim ${ }^{24 a}$, S.P. Marsden ${ }^{83}$, Z. Marshall ${ }^{15}$, L.F. Marti ${ }^{17}$,

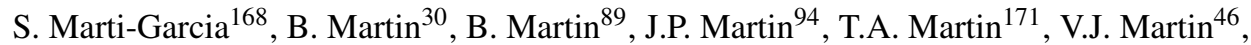
B. Martin dit Latour ${ }^{14}$, H. Martinez ${ }^{137}$, M. Martinez ${ }^{12, l}$, S. Martin-Haugh ${ }^{130}$, A.C. Martyniuk ${ }^{77}$, M. Marx ${ }^{139}$, F. Marzano ${ }^{133 a}$, A. Marzin ${ }^{30}$, L. Masetti ${ }^{82}$, T. Mashimo ${ }^{156}$, R. Mashinistov ${ }^{95}$, J. Masik ${ }^{83}$, A.L. Maslennikov ${ }^{108}$, I. Massa ${ }^{20 a, 20 b}$, N. Massol $^{5}$, P. Mastrandrea ${ }^{149}$, A. Mastroberardino ${ }^{37 a, 37 b}$, T. Masubuchi ${ }^{156}$, P. Matricon ${ }^{116}$, H. Matsunaga ${ }^{156}$, T. Matsushita ${ }^{66}$, P. Mättig ${ }^{176}$, S. Mättig ${ }^{42}$, J. Mattmann ${ }^{82}$, J. Maurer ${ }^{26 a}$, S.J. Maxfield ${ }^{73}$, D.A. Maximov ${ }^{108, p}$, R. Mazini ${ }^{152}$, L. Mazzaferro ${ }^{134 a, 134 b}$, G. Mc Goldrick ${ }^{159}$, S.P. Mc Kee ${ }^{88}$, A. McCarn ${ }^{88}$, R.L. McCarthy ${ }^{149}$, T.G. McCarthy ${ }^{29}$, N.A. McCubbin ${ }^{130}$, K.W. McFarlane ${ }^{56, *}$, J.A. Mcfayden ${ }^{77}$, G. Mchedlidze ${ }^{54}$, T. Mclaughlan ${ }^{18}$, S.J. McMahon ${ }^{130}$, R.A. McPherson ${ }^{170, h}$, A. Meade ${ }^{85}$, J. Mechnich ${ }^{106}$, M. Medinnis ${ }^{42}$, S. Meehan ${ }^{31}$, S. Mehlhase ${ }^{36}$, A. Mehta $^{73}$, K. Meier ${ }^{58 a}$, C. Meineck ${ }^{99}$, B. Meirose ${ }^{80}$, C. Melachrinos ${ }^{31}$, B.R. Mellado Garcia ${ }^{146 c}$, F. Meloni ${ }^{90 a, 90 b}$, A. Mengarelli ${ }^{20 a, 20 b}$, S. Menke ${ }^{100}$, E. Meoni ${ }^{162}$, K.M. Mercurio ${ }^{57}$, S. Mergelmeyer ${ }^{21}$, N. Meric ${ }^{137}$, P. Mermod ${ }^{49}$, L. Merola ${ }^{103 a, 103 b}$, C. Meroni ${ }^{90 a}$, F.S. Merritt ${ }^{31}$, H. Merritt ${ }^{110}$, A. Messina ${ }^{30, w}$, J. Metcalfe ${ }^{25}$, A.S. Mete ${ }^{164}$, C. Meyer ${ }^{82}$, C. Meyer ${ }^{31}$, J-P. Meyer ${ }^{137}$, J. Meyer ${ }^{30}$, R.P. Middleton ${ }^{130}$, S. Migas ${ }^{73}$, L. Mijović137, G. Mikenberg ${ }^{173}$, M. Mikestikova ${ }^{126}$, M. Mikuž ${ }^{74}$, D.W. Miller ${ }^{31}$, C. Mills ${ }^{46}$, A. Milov ${ }^{173}$, D.A. Milstead ${ }^{147 a, 147 b}$, D. Milstein ${ }^{173}$, A.A. Minaenko ${ }^{129}$, M. Miñano Moya ${ }^{168}$, I.A. Minashvili ${ }^{64}$, A.I. Mincer ${ }^{109}$, B. Mindur ${ }^{38 a}$, M. Mineev ${ }^{64}$, Y. Ming ${ }^{174}$, L.M. Mir ${ }^{12}$, G. Mirabelli ${ }^{133 a}$, T. Mitani ${ }^{172}$, J. Mitrevski ${ }^{99}$, V.A. Mitsou ${ }^{168}$, S. Mitsui ${ }^{65}$, A. Miucci $^{49}$, P.S. Miyagawa ${ }^{140}$, J.U. Mjörnmark ${ }^{80}$, T. Moa ${ }^{147 a, 147 b}$, K. Mochizuki ${ }^{84}$, V. Moeller ${ }^{28}$, S. Mohapatra ${ }^{35}$, W. Mohr ${ }^{48}$, S. Molander ${ }^{147 a, 147 b}$, R. Moles-Valls ${ }^{168}$, K. Mönig ${ }^{42}$, C. Monini5 ${ }^{55}$, J. Monk ${ }^{36}$, E. Monnier ${ }^{84}$, J. Montejo Berlingen ${ }^{12}$, F. Monticelli ${ }^{70}$, S. Monzani ${ }^{133 a, 133 b}$, R.W. Moore ${ }^{3}$, A. Moraes ${ }^{53}$, N. Morange ${ }^{62}$,

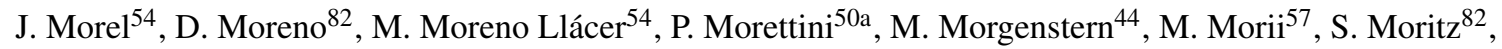
A.K. Morley ${ }^{148}$, G. Mornacchi ${ }^{30}$, J.D. Morris ${ }^{75}$, L. Morvaj ${ }^{102}$, H.G. Moser ${ }^{100}$, M. Mosidze ${ }^{51 b}$, J. Moss ${ }^{110}$, R. Mount ${ }^{144}$, E. Mountricha ${ }^{25}$, S.V. Mouraviev ${ }^{95, *}$, E.J.W. Moyse ${ }^{85}$, S. Muanza ${ }^{84}$, R.D. Mudd ${ }^{18}$, F. Mueller ${ }^{58 a}$, J. Mueller ${ }^{124}$, K. Mueller ${ }^{21}$, T. Mueller ${ }^{28}$, T. Mueller ${ }^{82}$, D. Muenstermann ${ }^{49}$, Y. Munwes ${ }^{154}$, J.A. Murillo Quijada ${ }^{18}$, W.J. Murray ${ }^{171,130}$, H. Musheghyan ${ }^{54}$, E. Musto ${ }^{153}$, A.G. Myagkov ${ }^{129, x}$, M. Myska ${ }^{127}$, O. Nackenhorst ${ }^{54}$, J. Nadal ${ }^{54}$, K. Nagai ${ }^{61}$, R. Nagai ${ }^{158}$, Y. Nagai ${ }^{84}$, K. Nagano ${ }^{65}$, A. Nagarkar ${ }^{110}$, Y. Nagasaka ${ }^{59}$, M. Nagel ${ }^{100}$, A.M. Nairz ${ }^{30}$, Y. Nakahama ${ }^{30}$, K. Nakamura ${ }^{65}$, T. Nakamura ${ }^{156}$, I. Nakano ${ }^{111}$, H. Namasivayam ${ }^{41}$, G. Nanava ${ }^{21}$, R. Narayan ${ }^{58 b}$, T. Nattermann ${ }^{21}$, T. Naumann ${ }^{42}$, G. Navarro ${ }^{163}$, R. Nayyar ${ }^{7}$, H.A. Neal ${ }^{88}$, P.Yu. Nechaeva ${ }^{95}$, T.J. Neep ${ }^{83}$, A. Negri ${ }^{120 a, 120 b}$, G. Negri $^{30}$, M. Negrini ${ }^{20 a}$, S. Nektarijevic ${ }^{49}$, A. Nelson ${ }^{164}$, T.K. Nelson ${ }^{144}$, S. Nemecek ${ }^{126}$, P. Nemethy ${ }^{109}$, 
A.A. Nepomuceno ${ }^{24 a}$, M. Nessi ${ }^{30, y}$, M.S. Neubauer ${ }^{166}$, M. Neumann ${ }^{176}$, R.M. Neves ${ }^{109}$, P. Nevski ${ }^{25}$, F.M. Newcomer ${ }^{121}$, P.R. Newman ${ }^{18}$, D.H. Nguyen ${ }^{6}$, R.B. Nickerson ${ }^{119}$, R. Nicolaidou ${ }^{137}$, B. Nicquevert ${ }^{30}$, J. Nielsen ${ }^{138}$, N. Nikiforou ${ }^{35}$, A. Nikiforov ${ }^{16}$, V. Nikolaenko ${ }^{129, x}$, I. Nikolic-Audit ${ }^{79}$, K. Nikolics ${ }^{49}$, K. Nikolopoulos ${ }^{18}$, P. Nilsson ${ }^{8}$, Y. Ninomiya ${ }^{156}$, A. Nisati ${ }^{133 a}$, R. Nisius ${ }^{100}$, T. Nobe ${ }^{158}$, L. Nodulman ${ }^{6}$,

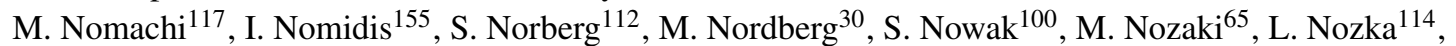
K. Ntekas ${ }^{10}$, G. Nunes Hanninger ${ }^{87}$, T. Nunnemann ${ }^{99}$, E. Nurse ${ }^{77}$, F. Nuti ${ }^{87}$, B.J. O'Brien ${ }^{46}$, F. O' grady $^{7}$, D.C. O'Neil ${ }^{143}$, V. O’Shea ${ }^{53}$, F.G. Oakham ${ }^{29, d}$, H. Oberlack ${ }^{100}$, T. Obermann ${ }^{21}$, J. Ocariz ${ }^{79}$, A. Ochi ${ }^{66}$, M.I. Ochoa ${ }^{77}$, S. Oda ${ }^{69}$, S. Odaka ${ }^{65}$, H. Ogren ${ }^{60}$, A. $\mathrm{Oh}^{83}$, S.H. Oh ${ }^{45}$, C.C. $\mathrm{Ohm}^{30}$, H. Ohman ${ }^{167}$, T. Ohshima ${ }^{102}$, W. Okamura ${ }^{117}$, H. Okawa ${ }^{25}$, Y. Okumura ${ }^{31}$, T. Okuyama ${ }^{156}$, A. Olariu ${ }^{26 a}$, A.G. Olchevski ${ }^{64}$, S.A. Olivares Pino ${ }^{46}$, D. Oliveira Damazio ${ }^{25}$, E. Oliver Garcia ${ }^{168}$, A. Olszewski ${ }^{39}$, J. Olszowska ${ }^{39}$, A. Onofre ${ }^{125 a, 125 e}$, P.U.E. Onyisi ${ }^{31, z}$, C.J. Oram ${ }^{160 a}$, M.J. Oreglia ${ }^{31}$, Y. Oren ${ }^{154}$, D. Orestano ${ }^{135 a, 135 b}$, N. Orlando ${ }^{72 a, 72 b}$, C. Oropeza Barrera ${ }^{53}$, R.S. Orr ${ }^{159}$, B. Osculati ${ }^{50 a, 50 b}$, R. Ospanov ${ }^{121}$, G. Otero y Garzon ${ }^{27}$, H. Otono ${ }^{69}$, M. Ouchrif ${ }^{136 d}$, E.A. Ouellette ${ }^{170}$, F. Ould-Saada ${ }^{118}$, A. Ouraou ${ }^{137}$, K.P. Oussoren ${ }^{106}$, Q. Ouyang ${ }^{33 a}$, A. Ovcharova ${ }^{15}$, M. Owen ${ }^{83}$, V.E. Ozcan ${ }^{19 a}$, N. Ozturk ${ }^{8}$, K. Pachal ${ }^{119}$, A. Pacheco Pages ${ }^{12}$, C. Padilla Aranda ${ }^{12}$, M. Pagáčová ${ }^{48}$, S. Pagan Griso ${ }^{15}$, E. Paganis ${ }^{140}$, C. Pahl ${ }^{100}$, F. Paige ${ }^{25}$, P. Pais ${ }^{85}$, K. Pajchel ${ }^{118}$, G. Palacino ${ }^{160 b}$, S. Palestini ${ }^{30}$, D. Pallin ${ }^{34}$, A. Palma ${ }^{125 a, 125 b}$, J.D. Palmer ${ }^{18}$, Y.B. Pan ${ }^{174}$, E. Panagiotopoulou ${ }^{10}$, J.G. Panduro Vazquez ${ }^{76}$, P. Pani ${ }^{106}$, N. Panikashvili ${ }^{88}$, S. Panitkin ${ }^{25}$, D. Pantea ${ }^{26 a}$, L. Paolozzi134a,134b ${ }^{13}$ Th.D. Papadopoulou ${ }^{10}$, K. Papageorgiou ${ }^{155, j}$, A. Paramonov ${ }^{6}$, D. Paredes Hernandez ${ }^{34}$, M.A. Parker ${ }^{28}$, F. Parodi ${ }^{50 a, 50 b}$, J.A. Parsons ${ }^{35}$, U. Parzefall ${ }^{48}$, E. Pasqualucci ${ }^{133 a}$, S. Passaggio ${ }^{50 a}$, A. Passeri $^{135 a}$, F. Pastore ${ }^{135 a, 135 b, *}$, Fr. Pastore ${ }^{76}$, G. Pásztor ${ }^{49, a a}$, S. Pataraia ${ }^{176}$, N.D. Patel ${ }^{151}$, J.R. Pater ${ }^{83}$, S. Patricelli ${ }^{103 a, 103 b}$, T. Pauly ${ }^{30}$, J. Pearce ${ }^{170}$, M. Pedersen ${ }^{118}$, S. Pedraza Lopez ${ }^{168}$, R. Pedro ${ }^{125 a, 125 b}$, S.V. Peleganchuk ${ }^{108}$, D. Pelikan ${ }^{167}$, H. Peng ${ }^{33 b}$, B. Penning ${ }^{31}$, J. Penwell ${ }^{60}$, D.V. Perepelitsa ${ }^{25}$, E. Perez Codina ${ }^{160 a}$, M.T. Pérez García-Estañ ${ }^{168}$, V. Perez Reale ${ }^{35}$, L. Perini ${ }^{90 a, 90 b}, H_{.}$Pernegger $^{30}$, R. Perrino ${ }^{72 a}$, R. Peschke ${ }^{42}$, V.D. Peshekhonov ${ }^{64}$, K. Peters ${ }^{30}$, R.F.Y. Peters ${ }^{83}$, B.A. Petersen ${ }^{87}$, J. Petersen ${ }^{30}$, T.C. Petersen ${ }^{36}$, E. Petit ${ }^{42}$, A. Petridis ${ }^{147 a, 147 b}$, C. Petridou ${ }^{155}$, E. Petrolo ${ }^{133 a}$, F. Petrucci ${ }^{135 a, 135 b}$, M. Petteni $^{143}$, N.E. Pettersson ${ }^{158}$, R. Pezoa ${ }^{32 b}$, P.W. Phillips ${ }^{130}$, G. Piacquadio ${ }^{144}$, E. Pianori ${ }^{171}$, A. Picazio ${ }^{49}$, E. Piccaro ${ }^{75}$, M. Piccinini ${ }^{20 a, 20 b}$, R. Piegaia ${ }^{27}$, J.P. Pieron ${ }^{61}$, D.T. Pignotti ${ }^{110}$, J.E. Pilcher $^{31}$, A.D. Pilkington ${ }^{77}$, J. Pina ${ }^{125 a, 125 b, 125 d}$, M. Pinamonti ${ }^{165 a, 165 c, a b}$, A. Pinder ${ }^{119}$, J.L. Pinfold ${ }^{3}$, A. Pingel ${ }^{36}$, B. Pinto ${ }^{125 a}$, S. Pires ${ }^{79}$, M. Pitt ${ }^{173}$, C. Pizio ${ }^{90 a, 90 b}$, M.-A. Pleier ${ }^{25}$, V. Pleskot ${ }^{128}$, E. Plotnikova ${ }^{64}$, P. Plucinski ${ }^{147 a, 147 b}$, S. Poddar ${ }^{58 a}$, F. Podlyski ${ }^{34}$, R. Poettgen ${ }^{82}$, L. Poggioli ${ }^{116}$, D. Pohl ${ }^{21}$, M. Pohl ${ }^{49}$, G. Polesello ${ }^{120 a}$, A. Policicchio ${ }^{37 a, 37 b}$, R. Polifka ${ }^{159}$, A. Polini ${ }^{20 a}$, C.S. Pollard ${ }^{45}$, V. Polychronakos ${ }^{25}$, K. Pommès ${ }^{30}$, L. Pontecorvo ${ }^{133 a}$, B.G. Pope ${ }^{89}$, G.A. Popeneciu ${ }^{26 b}$, D.S. Popovic ${ }^{13 a}$, A. Poppleton ${ }^{30}$, X. Portell Bueso ${ }^{12}$, G.E. Pospelov ${ }^{100}$, S. Pospisil ${ }^{127}$, K. Potamianos ${ }^{15}$, I.N. Potrap ${ }^{64}$, C.J. Potter ${ }^{150}$, C.T. Potter ${ }^{115}$,

G. Poulard ${ }^{30}$, J. Poveda ${ }^{60}$, V. Pozdnyakov ${ }^{64}$, P. Pralavorio ${ }^{84}$, A. Pranko ${ }^{15}$, S. Prasad ${ }^{30}$, R. Pravahan ${ }^{8}$, S. Prell ${ }^{63}$, D. Price ${ }^{83}$, J. Price ${ }^{73}$, L.E. Price ${ }^{6}$, D. Prieur ${ }^{124}$, M. Primavera ${ }^{72 a}$, M. Proissl ${ }^{46}$, K. Prokofiev ${ }^{47}$, F. Prokoshin ${ }^{32 b}$, E. Protopapadaki ${ }^{137}$, S. Protopopescu ${ }^{25}$, J. Proudfoot $^{6}$, M. Przybycien $^{38 a}$, H. Przysiezniak ${ }^{5}$, E. Ptacek ${ }^{115}$, E. Pueschel ${ }^{85}$, D. Puldon ${ }^{149}$, M. Purohit ${ }^{25}, a c$, P. Puzo ${ }^{116}$, J. Qian ${ }^{88}$, G. Qin ${ }^{53}$, Y. Qin ${ }^{83}$, A. Quadt ${ }^{54}$, D.R. Quarrie ${ }^{15}$, W.B. Quayle ${ }^{165 a, 165 b}$, D. Quilty ${ }^{53}$, A. Qureshi ${ }^{160 b}$, V. Radeka ${ }^{25}$, V. Radescu ${ }^{42}$, S.K. Radhakrishnan ${ }^{149}$, P. Radloff ${ }^{115}$, P. Rados ${ }^{87}$, F. Ragusa ${ }^{90 a, 90 b}$, G. Rahal ${ }^{179}$, S. Rajagopalan ${ }^{25}$, M. Rammensee ${ }^{30}$, A.S. Randle-Conde ${ }^{40}$, C. Rangel-Smith ${ }^{167}$, K. Rao ${ }^{164}$, F. Rauscher ${ }^{99}$, T.C. Rave $^{48}$, T. Ravenscroft ${ }^{53}$, M. Raymond ${ }^{30}$, A.L. Read ${ }^{118}$, D.M. Rebuzzi ${ }^{120 a, 120 b}$, A. Redelbach ${ }^{175}$, G. Redlinger ${ }^{25}$, R. Reece ${ }^{138}$, K. Reeves ${ }^{41}$, L. Rehnisch ${ }^{16}$, A. Reinsch ${ }^{115}$, H. Reisin ${ }^{27}$, M. Relich ${ }^{164}$, C. Rembser $^{30}$, Z.L. Ren ${ }^{152}$, A. Renaud ${ }^{116}$, M. Rescigno ${ }^{133 a}$, S. Resconi ${ }^{90 a}$, B. Resende ${ }^{137}$, O.L. Rezanova ${ }^{108, p}$, P. Reznicek ${ }^{128}$, R. Rezvani ${ }^{94}$, R. Richter ${ }^{100}$, M. Ridel ${ }^{79}$, P. Rieck ${ }^{16}$, M. Rijssenbeek ${ }^{149}$, A. Rimoldi ${ }^{120 a, 120 b}$, L. Rinaldi ${ }^{20 a}$, E. Ritsch ${ }^{61}$, I. Riu ${ }^{12}$, F. Rizatdinova ${ }^{113}$, E. Rizvi ${ }^{75}$, S.H. Robertson ${ }^{86, h}$, A. Robichaud-Veronneau ${ }^{119}$, D. Robinson ${ }^{28}$, J.E.M. Robinson ${ }^{83}$, A. Robson ${ }^{53}$, C. Roda ${ }^{123 a, 123 b}$, L. Rodrigues ${ }^{30}$, S. Roe ${ }^{30}$, O. Røhne ${ }^{118}$, S. Rolli ${ }^{162}$, A. Romaniouk ${ }^{97}$, M. Romano ${ }^{20 a, 20 b}$, 
G. Romeo ${ }^{27}$, E. Romero Adam ${ }^{168}$, N. Rompotis ${ }^{139}$, L. Roos ${ }^{79}$, E. Ros ${ }^{168}$, S. Rosati ${ }^{133 a}$, K. Rosbach ${ }^{49}$, M. Rose ${ }^{76}$, P.L. Rosendahl ${ }^{14}$, O. Rosenthal ${ }^{142}$, V. Rossetti ${ }^{147 a, 147 b}$, E. Rossi ${ }^{103 a, 103 b}$, L.P. Rossi ${ }^{50 a}$, R. Rosten ${ }^{139}$, M. Rotaru ${ }^{26 a}$, I. Roth ${ }^{173}$, J. Rothberg ${ }^{139}$, D. Rousseau ${ }^{116}$, C.R. Royon ${ }^{137}$, A. Rozanov ${ }^{84}$, Y. Rozen ${ }^{153}$, X. Ruan ${ }^{146 c}$, F. Rubbo ${ }^{12}$, I. Rubinskiy ${ }^{42}$, V.I. Rud ${ }^{98}$, C. Rudolph ${ }^{44}$, M.S. Rudolph ${ }^{159}$, F. Rühr ${ }^{48}$, A. Ruiz-Martinez ${ }^{30}$, Z. Rurikova ${ }^{48}$, N.A. Rusakovich ${ }^{64}$, A. Ruschke ${ }^{99}$, J.P. Rutherfoord ${ }^{7}$, N. Ruthmann ${ }^{48}$, Y.F. Ryabov ${ }^{122}$, M. Rybar ${ }^{128}$, G. Rybkin ${ }^{116}$, N.C. Ryder ${ }^{119}$, A.F. Saavedra ${ }^{151}$, S. Sacerdoti ${ }^{27}$, A. Saddique ${ }^{3}$, I. Sadeh ${ }^{154}$, H.F-W. Sadrozinski ${ }^{138}$, R. Sadykov ${ }^{64}$, F. Safai Tehrani ${ }^{133 a}$, H. Sakamoto ${ }^{156}$, Y. Sakurai ${ }^{172}$, G. Salamanna ${ }^{75}$, A. Salamon ${ }^{134 a}$, M. Saleem ${ }^{112}$, D. Salek ${ }^{106}$, P.H. Sales De Bruin ${ }^{139}$, D. Salihagic ${ }^{100}$, A. Salnikov ${ }^{144}$, J. Salt ${ }^{168}$, B.M. Salvachua Ferrando ${ }^{6}$, D. Salvatore ${ }^{37 a, 37 b}$, F. Salvatore ${ }^{150}$, A. Salvucci ${ }^{105}$, A. Salzburger ${ }^{30}$, D. Sampsonidis ${ }^{155}$,

A. Sanchez ${ }^{103 a, 103 b}$, J. Sánchez ${ }^{168}$, V. Sanchez Martinez ${ }^{168}$, H. Sandaker ${ }^{14}$, R.L. Sandbach ${ }^{75}$, H.G. Sander ${ }^{82}$, M.P. Sanders ${ }^{99}$, M. Sandhoff ${ }^{176}$, T. Sandoval ${ }^{28}$, C. Sandoval ${ }^{163}$, R. Sandstroem ${ }^{100}$, D.P.C. Sankey ${ }^{130}$, A. Sansoni ${ }^{47}$, C. Santoni ${ }^{34}$, R. Santonico ${ }^{134 a, 134 b}$, H. Santos ${ }^{125 a}$, I. Santoyo Castillo ${ }^{150}$, K. Sapp ${ }^{124}$, A. Sapronov ${ }^{64}$, J.G. Saraiva ${ }^{125 a, 125 d}$, B. Sarrazin ${ }^{21}$, G. Sartisohn ${ }^{176}$, O. Sasaki ${ }^{65}$, Y. Sasaki ${ }^{156}$, I. Satsounkevitch ${ }^{91}$, G. Sauvage ${ }^{5, *}$, E. Sauvan ${ }^{5}$, P. Savard ${ }^{159, d}$, D.O. Savu ${ }^{30}$, C. Sawyer ${ }^{119}$, L. Sawyer ${ }^{78, k}$, J. Saxon ${ }^{121}$, C. Sbarra ${ }^{20 a}$, A. Sbrizzi ${ }^{3}$, T. Scanlon ${ }^{30}$, D.A. Scannicchio ${ }^{164}$, M. Scarcella ${ }^{151}$, J. Schaarschmidt ${ }^{173}$, P. Schacht ${ }^{100}$, D. Schaefer ${ }^{121}$, R. Schaefer ${ }^{42}$, S. Schaepe ${ }^{21}$, S. Schaetzel ${ }^{58 b}$, U. Schäfer ${ }^{82}$, A.C. Schaffer ${ }^{116}$, D. Schaile ${ }^{99}$, R.D. Schamberger ${ }^{149}$, V. Scharf ${ }^{58 a}$, V.A. Schegelsky ${ }^{122}$, D. Scheirich ${ }^{128}$, M. Schernau ${ }^{164}$, M.I. Scherzer ${ }^{35}$, C. Schiavi ${ }^{50 a, 50 b}$, J. Schieck ${ }^{99}$, C. Schillo ${ }^{48}$, M. Schioppa ${ }^{37 a, 37 b}$, S. Schlenker ${ }^{30}$, E. Schmidt ${ }^{48}$, K. Schmieden ${ }^{30}$, C. Schmitt ${ }^{82}$, C. Schmitt ${ }^{99}$, S. Schmitt ${ }^{58 b}$, B. Schneider ${ }^{17}$, Y.J. Schnellbach ${ }^{73}$, U. Schnoor ${ }^{44}$, L. Schoeffel ${ }^{137}$, A. Schoening ${ }^{58 b}$, B.D. Schoenrock ${ }^{89}$, A.L.S. Schorlemmer ${ }^{54}$, M. Schott ${ }^{82}$, D. Schouten ${ }^{160 a}$, J. Schovancova ${ }^{25}$, M. Schram ${ }^{86}$, S. Schramm ${ }^{159}$, M. Schreyer ${ }^{175}$, C. Schroeder ${ }^{82}$, N. Schuh ${ }^{82}$, M.J. Schultens ${ }^{21}$, H.-C. Schultz-Coulon ${ }^{58 a}$, H. Schulz ${ }^{16}$, M. Schumacher ${ }^{48}$, B.A. Schumm ${ }^{138}$, Ph. Schune ${ }^{137}$, A. Schwartzman ${ }^{144}$, Ph. Schwegler ${ }^{100}$, Ph. Schwemling ${ }^{137}$, R. Schwienhorst ${ }^{89}$, J. Schwindling ${ }^{137}$, T. Schwindt ${ }^{21}$, M. Schwoerer ${ }^{5}$, F.G. Sciacca ${ }^{17}$, E. Scifo ${ }^{116}$, G. Sciolla ${ }^{23}$, W.G. Scott ${ }^{130}$, F. Scuri ${ }^{123 a, 123 b}$, F. Scutti ${ }^{21}$, J. Searcy ${ }^{88}$, G. Sedov ${ }^{42}$, E. Sedykh ${ }^{122}$, S.C. Seidel ${ }^{104}$, A. Seiden ${ }^{138}$, F. Seifert ${ }^{127}$, J.M. Seixas ${ }^{24 a}$, G. Sekhniaidze ${ }^{103 a}$, S.J. Sekula ${ }^{40}$, K.E. Selbach ${ }^{46}$, D.M. Seliverstov ${ }^{122, *}$, G. Sellers ${ }^{73}$, N. Semprini-Cesari ${ }^{20 a, 20 b}$, C. Serfon ${ }^{30}$, L. Serin ${ }^{116}$, L. Serkin ${ }^{54}$, T. Serre ${ }^{84}$, R. Seuster ${ }^{160 a}$, H. Severini ${ }^{112}$, F. Sforza ${ }^{100}$, A. Sfyrla ${ }^{30}$, E. Shabalina ${ }^{54}$, M. Shamim ${ }^{115}$, L.Y. Shan ${ }^{33 a}$, J.T. Shank ${ }^{22}$, Q.T. Shao ${ }^{87}$, M. Shapiro ${ }^{15}$, P.B. Shatalov ${ }^{96}$, K. Shaw ${ }^{165 a, 165 b}$ R.S. Shaw ${ }^{28}$, P. Sherwood ${ }^{77}$, S. Shimizu ${ }^{66}$, C.O. Shimmin ${ }^{164}$, M. Shimojima ${ }^{101}$, M. Shiyakova ${ }^{64}$, A. Shmeleva ${ }^{95}$, M.J. Shochet ${ }^{31}$, D. Short ${ }^{119}$, S. Shrestha ${ }^{63}$, E. Shulga ${ }^{97}$, M.A. Shupe ${ }^{7}$, S. Shushkevich ${ }^{42}$, P. Sicho ${ }^{126}$, D. Sidorov ${ }^{113}$, A. Sidoti ${ }^{133 a}$, F. Siegert ${ }^{44}$, Dj. Sijacki ${ }^{13 a}$, O. Silbert ${ }^{173}$, J. Silva ${ }^{125 a, 125 d}$, Y. Silver ${ }^{154}$, D. Silverstein ${ }^{144}$, S.B. Silverstein ${ }^{147 a}$, V. Simak ${ }^{127}$, O. Simard ${ }^{5}$, Lj. Simic ${ }^{13 a}$, S. Simion ${ }^{116}$, E. Simioni ${ }^{82}$, B. Simmons ${ }^{77}$, R. Simoniello ${ }^{90 a, 90 b}$, M. Simonyan ${ }^{36}$, P. Sinervo ${ }^{159}$, N.B. Sinev ${ }^{115}$, V. Sipica ${ }^{142}$, G. Siragusa ${ }^{175}$, A. Sircar ${ }^{78}$, A.N. Sisakyan ${ }^{64, *}$, S.Yu. Sivoklokov ${ }^{98}$, J. Sjölin ${ }^{147 a, 147 b}$, T.B. Sjursen ${ }^{14}$, H.P. Skottowe ${ }^{57}$, K.Yu. Skovpen ${ }^{108}$, P. Skubic ${ }^{112}$, M. Slater ${ }^{18}$, T. Slavicek ${ }^{127}$, K. Sliwa ${ }^{162}$, V. Smakhtin ${ }^{173}$, B.H. Smart ${ }^{46}$, L. Smestad ${ }^{14}$, S.Yu. Smirnov ${ }^{97}$, Y. Smirnov ${ }^{97}$, L.N. Smirnova ${ }^{98, a d}$, O. Smirnova ${ }^{80}$, M. Smizanska ${ }^{71}$, K. Smolek ${ }^{127}$, A.A. Snesarev ${ }^{95}$, G. Snidero ${ }^{75}$, J. Snow ${ }^{112}$, S. Snyder ${ }^{25}$, R. Sobie ${ }^{170, h}$, F. Socher ${ }^{44}$, J. Sodomka ${ }^{127}$, A. Soffer ${ }^{154}$, D.A. Soh ${ }^{152, s}$, C.A. Solans ${ }^{30}$, M. Solar ${ }^{127}$, J. Solc ${ }^{127}$, E.Yu. Soldatov ${ }^{97}$, U. Soldevila ${ }^{168}$, E. Solfaroli Camillocci ${ }^{133 a, 133 b}$, A.A. Solodkov ${ }^{129}$, O.V. Solovyanov ${ }^{129}$, V. Solovyev ${ }^{122}$, P. Sommer ${ }^{48}$, H.Y. Song ${ }^{33 b}$, N. Soni ${ }^{1}$, A. Sood ${ }^{15}$, A. Sopczak ${ }^{127}$, V. Sopko ${ }^{127}$, B. Sopko ${ }^{127}$, V. Sorin ${ }^{12}$, M. Sosebee ${ }^{8}$, R. Soualah ${ }^{165 a, 165 c}$, P. Soueid ${ }^{94}$, A.M. Soukharev ${ }^{108}$,

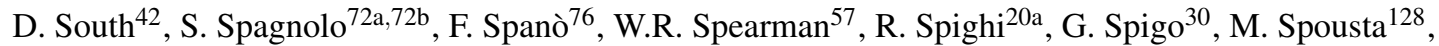
T. Spreitzer ${ }^{159}$, B. Spurlock ${ }^{8}$, R.D. St. Denis ${ }^{53}$, S. Staerz ${ }^{44}$, J. Stahlman ${ }^{121}$, R. Stamen ${ }^{58 a}$, E. Stanecka ${ }^{39}$, R.W. Stanek ${ }^{6}$, C. Stanescu ${ }^{135 a}$, M. Stanescu-Bellu ${ }^{42}$, M.M. Stanitzki ${ }^{42}$, S. Stapnes ${ }^{118}$, E.A. Starchenko ${ }^{129}$, J. Stark ${ }^{55}$, P. Staroba ${ }^{126}$, P. Starovoitov ${ }^{42}$, R. Staszewski ${ }^{39}$, P. Stavina ${ }^{145 a, *}$, G. Steele ${ }^{53}$, P. Steinberg ${ }^{25}$, I. Stekl ${ }^{127}$, B. Stelzer ${ }^{143}$, H.J. Stelzer ${ }^{30}$, O. Stelzer-Chilton ${ }^{160 a}$, H. Stenzel ${ }^{52}$, S. Stern ${ }^{100}$, G.A. Stewart ${ }^{53}$, 
J.A. Stillings ${ }^{21}$, M.C. Stockton ${ }^{86}$, M. Stoebe ${ }^{86}$, G. Stoicea ${ }^{26 a}$, P. Stolte ${ }^{54}$, S. Stonjek ${ }^{100}$, A.R. Stradling ${ }^{8}$, A. Straessner ${ }^{44}$, M.E. Stramaglia ${ }^{17}$, J. Strandberg ${ }^{148}$, S. Strandberg ${ }^{147 a, 147 b}$, A. Strandlie ${ }^{118}$, E. Strauss ${ }^{144}$, M. Strauss ${ }^{112}$, P. Strizenec ${ }^{145 b}$, R. Ströhmer ${ }^{175}$, D.M. Strom ${ }^{115}$, R. Stroynowski ${ }^{40}$, S.A. Stucci ${ }^{17}$, B. Stugu ${ }^{14}$, N.A. Styles ${ }^{42}$, D. Su ${ }^{144}$, J. Su${ }^{124}$, HS. Subramania ${ }^{3}$, R. Subramaniam ${ }^{78}$, A. Succurro ${ }^{12}$, Y. Sugaya ${ }^{117}$, C. Suhr ${ }^{107}$, M. Suk ${ }^{127}$, V.V. Sulin ${ }^{95}$, S. Sultansoy ${ }^{4 c}$, T. Sumida ${ }^{67}$, X. Sun ${ }^{33 a}$, J.E. Sundermann ${ }^{48}$, K. Suruliz ${ }^{140}$, G. Susinno ${ }^{37 a, 37 b}$, M.R. Sutton ${ }^{150}$, Y. Suzuki ${ }^{65}$, M. Svatos ${ }^{126}$, S. Swedish ${ }^{169}$, M. Swiatlowski ${ }^{144}$, I. Sykora ${ }^{145 a}$, T. Sykora ${ }^{128}$, D. Ta ${ }^{89}$, K. Tackmann ${ }^{42}$, J. Taenzer ${ }^{159}$, A. Taffard ${ }^{164}$, R. Tafirout ${ }^{160 a}$, N. Taiblum ${ }^{154}$, Y. Takahashi ${ }^{102}$, H. Takai ${ }^{25}$, R. Takashima ${ }^{68}$, H. Takeda $^{66}$, T. Takeshita ${ }^{141}$, Y. Takubo ${ }^{65}$, M. Talby ${ }^{84}$, A.A. Talyshev ${ }^{108, p}$, J.Y.C. Tam ${ }^{175}$, M.C. Tamsett $^{78, a e}$, K.G. Tan $^{87}$, J. Tanaka ${ }^{156}$, R. Tanaka ${ }^{116}$, S. Tanaka ${ }^{132}$, S. Tanaka ${ }^{65}$, A.J. Tanasijczuk ${ }^{143}$, K. Tani ${ }^{66}$,

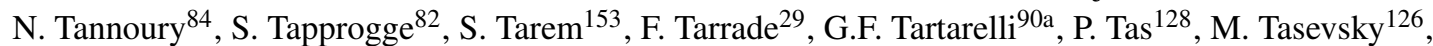
T. Tashiro ${ }^{67}$, E. Tassi ${ }^{37 a, 37 b}$, A. Tavares Delgado ${ }^{125 a, 125 b}$, Y. Tayalati ${ }^{136 d}$, F.E. Taylor ${ }^{93}$, G.N. Taylor ${ }^{87}$, W. Taylor ${ }^{160 b}$, F.A. Teischinger ${ }^{30}$, M. Teixeira Dias Castanheira ${ }^{75}$, P. Teixeira-Dias ${ }^{76}$, K.K. Temming ${ }^{48}$, H. Ten Kate ${ }^{30}$, P.K. Teng ${ }^{152}$, S. Terada ${ }^{65}$, K. Terashi ${ }^{156}$, J. Terron ${ }^{81}$, S. Terzo ${ }^{100}$, M. Testa ${ }^{47}$, R.J. Teuscher ${ }^{159, h}$, J. Therhaag ${ }^{21}$, T. Theveneaux-Pelzer ${ }^{34}$, S. Thoma ${ }^{48}$, J.P. Thomas ${ }^{18}$, J. Thomas-Wilsker ${ }^{76}$, E.N. Thompson ${ }^{35}$, P.D. Thompson ${ }^{18}$, P.D. Thompson ${ }^{159}$, A.S. Thompson ${ }^{53}$, L.A. Thomsen ${ }^{36}$, E. Thomson ${ }^{121}$, M. Thomson ${ }^{28}$, W.M. Thong ${ }^{87}$, R.P. Thun ${ }^{88, *}$, F. Tian ${ }^{35}$, M.J. Tibbetts ${ }^{15}$, V.O. Tikhomirov ${ }^{95, a f}$, Yu.A. Tikhonov ${ }^{108, p}$, S. Timoshenko ${ }^{97}$, E. Tiouchichine ${ }^{84}$, P. Tipton ${ }^{177}$, S. Tisserant ${ }^{84}$, T. Todorov ${ }^{5}$, S. Todorova-Nova ${ }^{128}$, B. Toggerson ${ }^{7}$, J. Tojo ${ }^{69}$, S. Tokár $^{145 a}$, K. Tokushuku ${ }^{65}$, K. Tollefson ${ }^{89}$, L. Tomlinson ${ }^{83}$, M. Tomoto ${ }^{102}$, L. Tompkins ${ }^{31}$, K. Toms ${ }^{104}$, N.D. Topilin ${ }^{64}$, E. Torrence ${ }^{115}$, H. Torres ${ }^{143}$, E. Torró Pastor ${ }^{168}$, J. Toth ${ }^{84, a a}$, F. Touchard ${ }^{84}$, D.R. Tovey ${ }^{140}$, H.L. Tran $^{116}$, T. Trefzger ${ }^{175}$,

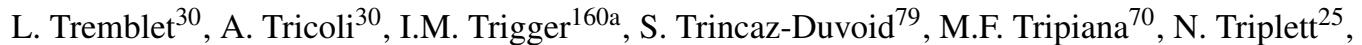

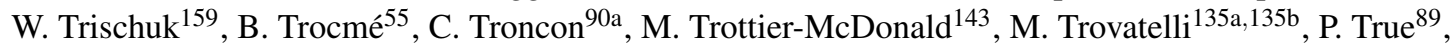
M. Trzebinski ${ }^{39}$, A. Trzupek $^{39}$, C. Tsarouchas ${ }^{30}$, J.C-L. Tseng ${ }^{119}$, P.V. Tsiareshka ${ }^{91}$, D. Tsionou ${ }^{137}$, G. Tsipolitis ${ }^{10}$, N. Tsirintanis ${ }^{9}$, S. Tsiskaridze ${ }^{12}$, V. Tsiskaridze ${ }^{48}$, E.G. Tskhadadze ${ }^{51 a}$, I.I. Tsukerman ${ }^{96}$, V. Tsulaia ${ }^{15}, \mathrm{~S}$. Tsuno ${ }^{65}$, D. Tsybychev ${ }^{149}$, A. Tudorache ${ }^{26 a}$, V. Tudorache ${ }^{26 a}$, A.N. Tuna ${ }^{121}$, S.A. Tupputi ${ }^{20 a, 20 b}$, S. Turchikhin ${ }^{98, a d}$, D. Turecek ${ }^{127}$, I. Turk Cakir ${ }^{4 d}$, R. Turra90a,90b , P.M. Tuts ${ }^{35}$,

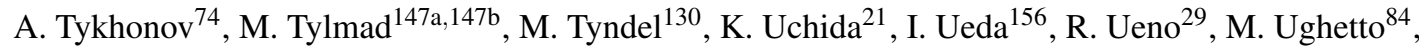

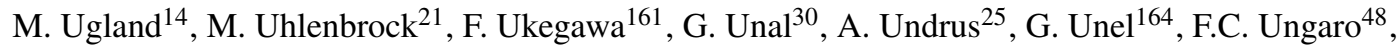
Y. Unno ${ }^{65}$, D. Urbaniec ${ }^{35}$, P. Urquijo ${ }^{21}$, G. Usai ${ }^{8}$, A. Usanova ${ }^{61}$, L. Vacavant ${ }^{84}$, V. Vacek ${ }^{127}$, B. Vachon ${ }^{86}$, N. Valencic ${ }^{106}, \mathrm{~S}$. Valentinetti ${ }^{20 \mathrm{a}, 20 \mathrm{~b}}, \mathrm{~A}$. Valero ${ }^{168}$, L. Valery ${ }^{34}, \mathrm{~S}$. Valkar ${ }^{128}$, E. Valladolid Gallego ${ }^{168}$, S. Vallecorsa ${ }^{49}$, J.A. Valls Ferrer ${ }^{168}$, R. Van Berg ${ }^{121}$, P.C. Van Der Deijl ${ }^{106}$, R. van der Geer ${ }^{106}$, H. van der Graaf ${ }^{106}$, R. Van Der Leeuw ${ }^{106}$, D. van der $\mathrm{Ster}^{30}$, N. van $\mathrm{Eldik}^{30}$, P. van Gemmeren ${ }^{6}$, J. Van Nieuwkoop ${ }^{143}$, I. van Vulpen ${ }^{106}$, M.C. van Woerden $^{30}$, M. Vanadia ${ }^{133 a, 133 b}$, W. Vandelli ${ }^{30}$, R. Vanguri ${ }^{121}$, A. Vaniachine ${ }^{6}$, P. Vankov ${ }^{42}$, F. Vannucci ${ }^{79}$, G. Vardanyan ${ }^{178}$, R. Vari ${ }^{133 a}$, E.W. Varnes ${ }^{7}$, T. Varol ${ }^{85}$, D. Varouchas ${ }^{79}$, A. Vartapetian ${ }^{8}$, K.E. Varvell ${ }^{151}$, F. Vazeille ${ }^{34}$, T. Vazquez Schroeder ${ }^{54}$,

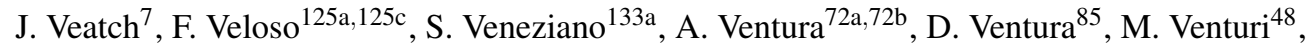
N. Venturi ${ }^{159}$, A. Venturini ${ }^{23}$, V. Vercesi ${ }^{120 a}$, M. Verducci ${ }^{139}$, W. Verkerke ${ }^{106}$, J.C. Vermeulen ${ }^{106}$, A. Vest $^{44}$, M.C. Vetterli ${ }^{143, d}$, O. Viazlo ${ }^{80}$, I. Vichou ${ }^{166}$, T. Vickey ${ }^{146 c, a g}$, O.E. Vickey Boeriu ${ }^{146 c}$,

G.H.A. Viehhauser ${ }^{19}$, S. Viel ${ }^{169}$, R. Vigne ${ }^{30}$, M. Villa ${ }^{20 a, 20 b}$, M. Villaplana Perez ${ }^{168}$, E. Vilucchi ${ }^{47}$, M.G. Vincter ${ }^{29}$, V.B. Vinogradov ${ }^{64}$, J. Virzi ${ }^{15}$, I. Vivarelli ${ }^{150}$, F. Vives Vaque ${ }^{3}$, S. Vlachos ${ }^{10}$, D. Vladoiu ${ }^{99}$,

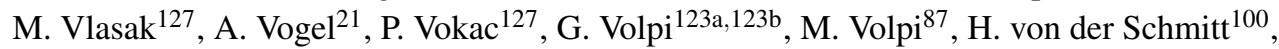
H. von Radziewski ${ }^{48}$, E. von Toerne ${ }^{21}$, V. Vorobel ${ }^{128}$, K. Vorobev $^{97}$, M. Vos ${ }^{168}$, R. Voss $^{30}$, J.H. Vossebeld ${ }^{73}$, N. Vranjes ${ }^{137}$, M. Vranjes Milosavljevic ${ }^{106}$, V. Vrba ${ }^{126}$, M. Vreeswijk ${ }^{106}$, T. Vu Anh ${ }^{48}$, R. Vuillermet ${ }^{30}$, I. Vukotic ${ }^{31}$, Z. Vykydal ${ }^{127}$, W. Wagner ${ }^{176}$, P. Wagner ${ }^{21}$, S. Wahrmund ${ }^{44}$, J. Wakabayashi ${ }^{102}$, J. Walder ${ }^{71}$, R. Walker ${ }^{99}$, W. Walkowiak ${ }^{142}$, R. Wall ${ }^{177}$, P. Waller $^{73}$, B. Walsh ${ }^{177}$, C. Wang ${ }^{152, a h}$, C. Wang ${ }^{45}$, F. Wang ${ }^{174}$, H. Wang ${ }^{15}$, H. Wang ${ }^{40}$, J. Wang ${ }^{42}$, J. Wang ${ }^{33 a}$, K. Wang ${ }^{86}$, R. Wang ${ }^{104}$, S.M. Wang ${ }^{152}$, T. Wang ${ }^{21}$, X. Wang ${ }^{177}$, C. Wanotayaroj ${ }^{115}$, A. Warburton ${ }^{86}$, C.P. Ward ${ }^{28}$, 
D.R. Wardrope ${ }^{77}$, M. Warren ${ }^{77}$, M. Warsinsky ${ }^{48}$, A. Washbrook ${ }^{46}$, C. Wasicki ${ }^{42}$, I. Watanabe $^{66}$, P.M. Watkins ${ }^{18}$, A.T. Watson ${ }^{18}$, I.J. Watson ${ }^{151}$, M.F. Watson ${ }^{18}$, G. Watts ${ }^{139}$, S. Watts ${ }^{83}$, B.M. Waugh ${ }^{77}$, S. Webb ${ }^{83}$, M.S. Weber ${ }^{17}$, S.W. Weber ${ }^{175}$, J.S. Webster ${ }^{31}$, A.R. Weidberg ${ }^{119}$, P. Weigell ${ }^{100}$, B. Weinert ${ }^{60}$, J. Weingarten ${ }^{54}$, C. Weiser ${ }^{48}$, H. Weits ${ }^{106}$, P.S. Wells ${ }^{30}$, T. Wenaus ${ }^{25}$, D. Wendland ${ }^{16}$, Z. Weng ${ }^{152, s}$, T. Wengler ${ }^{30}$, S. Wenig ${ }^{30}$, N. Wermes ${ }^{21}$, M. Werner ${ }^{48}$, P. Werner ${ }^{30}$, M. Wessels ${ }^{58 a}$, J. Wetter ${ }^{162}$, K. Whalen ${ }^{29}$, A. White ${ }^{8}$, M.J. White ${ }^{1}$, R. White ${ }^{32 b}$, S. White ${ }^{123 a, 123 b}$, D. Whiteson ${ }^{164}$, D. Wicke ${ }^{176}$, F.J. Wickens ${ }^{130}$, W. Wiedenmann ${ }^{174}$, M. Wielers ${ }^{130}$, P. Wienemann ${ }^{21}$, C. Wiglesworth ${ }^{36}$,

L.A.M. Wiik-Fuchs ${ }^{21}$, P.A. Wijeratne ${ }^{77}$, A. Wildauer ${ }^{100}$, M.A. Wildt ${ }^{42, a i}$, H.G. Wilkens ${ }^{30}$, J.Z. Will ${ }^{99}$, H.H. Williams ${ }^{121}$, S. Williams ${ }^{28}$, C. Willis ${ }^{89}$, S. Willocq ${ }^{85}$, J.A. Wilson ${ }^{18}$, A. Wilson ${ }^{88}$, I. Wingerter-Seez ${ }^{5}$, F. Winklmeier ${ }^{115}$, M. Wittgen ${ }^{144}$, T. Wittig ${ }^{43}$, J. Wittkowski ${ }^{99}$, S.J. Wollstadt ${ }^{82}$, M.W. Wolter ${ }^{39}$, H. Wolters ${ }^{125 a, 125 c}$, B.K. Wosiek ${ }^{39}$, J. Wotschack ${ }^{30}$, M.J. Woudstra ${ }^{83}$, K.W. Wozniak ${ }^{39}$, M. Wright ${ }^{53}$, M. $\mathrm{Wu}^{55}$, S.L. Wu ${ }^{174}$, X. Wu ${ }^{49}$, Y. Wu ${ }^{88}$, E. Wulf ${ }^{35}$, T.R. Wyatt ${ }^{83}$, B.M. Wynne ${ }^{46}$, S. Xella ${ }^{36}$, M. Xiao $^{137}$, D. Xu ${ }^{33 a}$, L. Xu ${ }^{33 b, a j}$, B. Yabsley ${ }^{151}$, S. Yacoob ${ }^{146 b, a k}$, M. Yamada ${ }^{65}$, H. Yamaguchi ${ }^{156}$, Y. Yamaguchi ${ }^{156}$, A. Yamamoto ${ }^{65}$, K. Yamamoto ${ }^{63}$, S. Yamamoto ${ }^{156}$, T. Yamamura ${ }^{156}$, T. Yamanaka ${ }^{156}$, K. Yamauchi ${ }^{102}$,

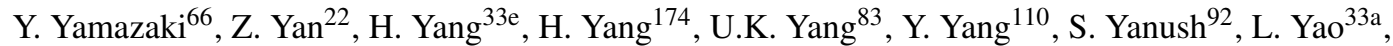
W-M. Yao ${ }^{15}$, Y. Yasu ${ }^{65}$, E. Yatsenko ${ }^{42}$, K.H. Yau Wong ${ }^{21}$, J. Ye ${ }^{40}$, S. Ye ${ }^{25}$, A.L. Yen ${ }^{57}$, E. Yildirim ${ }^{42}$, M. Yilmaz ${ }^{4 b}$, R. Yoosoofmiya ${ }^{124}$, K. Yorita ${ }^{172}$, R. Yoshida $^{6}$, K. Yoshihara ${ }^{156}$, C. Young ${ }^{144}$, C.J.S. Young ${ }^{30}$, S. Youssef ${ }^{22}$, D.R. Yu ${ }^{15}$, J. Yu ${ }^{8}$, J.M. Yu ${ }^{88}$, J. Yu ${ }^{113}$, L. Yuan ${ }^{66}$, A. Yurkewicz ${ }^{107}$, B. Zabinski ${ }^{39}$, R. Zaidan ${ }^{62}$, A.M. Zaitsev ${ }^{129, x}$, A. Zaman ${ }^{149}$, S. Zambito ${ }^{23}$, L. Zanello ${ }^{133 a, 133 b}$, D. Zanzi ${ }^{100}$, A. Zaytsev ${ }^{25}$, C. Zeitnitz ${ }^{176}$, M. Zeman ${ }^{127}$, A. Zemla ${ }^{38 a}$, K. Zengel ${ }^{23}$, O. Zenin ${ }^{129}$, T. Ženišr ${ }^{145 a}$, D. Zerwas ${ }^{116}$, G. Zevi della Porta ${ }^{57}$, D. Zhang ${ }^{88}$, F. Zhang ${ }^{174}$, H. Zhang ${ }^{89}$, J. Zhang ${ }^{6}$, L. Zhang ${ }^{152}$, X. Zhang ${ }^{33 d}$, Z. Zhang ${ }^{116}$, Z. Zhao ${ }^{33 b}$, A. Zhemchugov ${ }^{64}$, J. Zhong ${ }^{119}$, B. Zhou ${ }^{88}$, L. Zhou ${ }^{35}$, N. Zhou ${ }^{164}$, C.G. Zhu ${ }^{33 d}$, H. Zhu ${ }^{33 a}$, J. Zhu ${ }^{88}$, Y. Zhu ${ }^{33 b}$, X. Zhuang ${ }^{33 a}$, A. Zibell ${ }^{175}$, D. Zieminska ${ }^{60}$, N.I. Zimine ${ }^{64}$,

C. Zimmermann ${ }^{82}$, R. Zimmermann ${ }^{21}$, S. Zimmermann ${ }^{21}$, S. Zimmermann ${ }^{48}$, Z. Zinonos ${ }^{54}$, M. Ziolkowski ${ }^{142}$, G. Zobernig ${ }^{174}$, A. Zoccoli ${ }^{20 a, 20 b}$, M. zur Nedden ${ }^{16}$, G. Zurzolo $^{103 a, 103 b}$, V. Zutshi ${ }^{107}$, L. Zwalinski ${ }^{30}$.

1 Department of Physics, University of Adelaide, Adelaide, Australia

2 Physics Department, SUNY Albany, Albany NY, United States of America

3 Department of Physics, University of Alberta, Edmonton AB, Canada

4 (a) Department of Physics, Ankara University, Ankara; ${ }^{(b)}$ Department of Physics, Gazi University, Ankara; $(c)$ Division of Physics, TOBB University of Economics and Technology, Ankara; ${ }^{(d)}$ Turkish Atomic Energy Authority, Ankara, Turkey

5 LAPP, CNRS/IN2P3 and Université de Savoie, Annecy-le-Vieux, France

6 High Energy Physics Division, Argonne National Laboratory, Argonne IL, United States of America

7 Department of Physics, University of Arizona, Tucson AZ, United States of America

8 Department of Physics, The University of Texas at Arlington, Arlington TX, United States of America

9 Physics Department, University of Athens, Athens, Greece

10 Physics Department, National Technical University of Athens, Zografou, Greece

11 Institute of Physics, Azerbaijan Academy of Sciences, Baku, Azerbaijan

12 Institut de Física d'Altes Energies and Departament de Física de la Universitat Autònoma de Barcelona, Barcelona, Spain

13 (a) Institute of Physics, University of Belgrade, Belgrade; (b) Vinca Institute of Nuclear Sciences, University of Belgrade, Belgrade, Serbia

14 Department for Physics and Technology, University of Bergen, Bergen, Norway

15 Physics Division, Lawrence Berkeley National Laboratory and University of California, Berkeley CA, United States of America

16 Department of Physics, Humboldt University, Berlin, Germany

17 Albert Einstein Center for Fundamental Physics and Laboratory for High Energy Physics, University of Bern, Bern, Switzerland 
18 School of Physics and Astronomy, University of Birmingham, Birmingham, United Kingdom

19 (a) Department of Physics, Bogazici University, Istanbul; (b) Department of Physics, Dogus University, Istanbul; ${ }^{(c)}$ Department of Physics Engineering, Gaziantep University, Gaziantep, Turkey

$20{ }^{(a)}$ INFN Sezione di Bologna; ${ }^{(b)}$ Dipartimento di Fisica e Astronomia, Università di Bologna, Bologna, Italy

21 Physikalisches Institut, University of Bonn, Bonn, Germany

22 Department of Physics, Boston University, Boston MA, United States of America

23 Department of Physics, Brandeis University, Waltham MA, United States of America

24 (a) Universidade Federal do Rio De Janeiro COPPE/EE/IF, Rio de Janeiro; ${ }^{(b)}$ Federal University of Juiz de Fora (UFJF), Juiz de Fora; ${ }^{(c)}$ Federal University of Sao Joao del Rei (UFSJ), Sao Joao del Rei; ${ }^{(d)}$ Instituto de Fisica, Universidade de Sao Paulo, Sao Paulo, Brazil

25 Physics Department, Brookhaven National Laboratory, Upton NY, United States of America

26 (a) National Institute of Physics and Nuclear Engineering, Bucharest; ${ }^{(b)}$ National Institute for Research and Development of Isotopic and Molecular Technologies, Physics Department, Cluj Napoca; ${ }^{(c)}$ University Politehnica Bucharest, Bucharest; ${ }^{(d)}$ West University in Timisoara, Timisoara, Romania

27 Departamento de Física, Universidad de Buenos Aires, Buenos Aires, Argentina

28 Cavendish Laboratory, University of Cambridge, Cambridge, United Kingdom

29 Department of Physics, Carleton University, Ottawa ON, Canada

30 CERN, Geneva, Switzerland

31 Enrico Fermi Institute, University of Chicago, Chicago IL, United States of America

32 (a) Departamento de Física, Pontificia Universidad Católica de Chile, Santiago; ${ }^{(b)}$ Departamento de Física, Universidad Técnica Federico Santa María, Valparaíso, Chile

33 (a) Institute of High Energy Physics, Chinese Academy of Sciences, Beijing; ${ }^{(b)}$ Department of Modern Physics, University of Science and Technology of China, Anhui; ${ }^{(c)}$ Department of Physics, Nanjing University, Jiangsu; ${ }^{(d)}$ School of Physics, Shandong University, Shandong; ${ }^{(e)}$ Physics Department, Shanghai Jiao Tong University, Shanghai, China

34 Laboratoire de Physique Corpusculaire, Clermont Université and Université Blaise Pascal and CNRS/IN2P3, Clermont-Ferrand, France

35 Nevis Laboratory, Columbia University, Irvington NY, United States of America

36 Niels Bohr Institute, University of Copenhagen, Kobenhavn, Denmark

37 (a) INFN Gruppo Collegato di Cosenza, Laboratori Nazionali di Frascati; ${ }^{(b)}$ Dipartimento di Fisica, Università della Calabria, Rende, Italy

38 (a) AGH University of Science and Technology, Faculty of Physics and Applied Computer Science, Krakow; ${ }^{(b)}$ Marian Smoluchowski Institute of Physics, Jagiellonian University, Krakow, Poland

39 The Henryk Niewodniczanski Institute of Nuclear Physics, Polish Academy of Sciences, Krakow, Poland

40 Physics Department, Southern Methodist University, Dallas TX, United States of America

41 Physics Department, University of Texas at Dallas, Richardson TX, United States of America

42 DESY, Hamburg and Zeuthen, Germany

43 Institut für Experimentelle Physik IV, Technische Universität Dortmund, Dortmund, Germany

44 Institut für Kern- und Teilchenphysik, Technische Universität Dresden, Dresden, Germany

45 Department of Physics, Duke University, Durham NC, United States of America

46 SUPA - School of Physics and Astronomy, University of Edinburgh, Edinburgh, United Kingdom

47 INFN Laboratori Nazionali di Frascati, Frascati, Italy

48 Fakultät für Mathematik und Physik, Albert-Ludwigs-Universität, Freiburg, Germany

49 Section de Physique, Université de Genève, Geneva, Switzerland

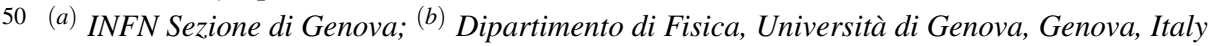

51 (a) E. Andronikashvili Institute of Physics, Iv. Javakhishvili Tbilisi State University, Tbilisi; ${ }^{(b)}$ High Energy Physics Institute, Tbilisi State University, Tbilisi, Georgia

52 II Physikalisches Institut, Justus-Liebig-Universität Giessen, Giessen, Germany

53 SUPA - School of Physics and Astronomy, University of Glasgow, Glasgow, United Kingdom

54 II Physikalisches Institut, Georg-August-Universität, Göttingen, Germany

55 Laboratoire de Physique Subatomique et de Cosmologie, Université Grenoble-Alpes, CNRS/IN2P3, Grenoble, France 
Department of Physics, Hampton University, Hampton VA, United States of America

57 Laboratory for Particle Physics and Cosmology, Harvard University, Cambridge MA, United States of America

58 (a) Kirchhoff-Institut für Physik, Ruprecht-Karls-Universität Heidelberg, Heidelberg; ${ }^{(b)}$ Physikalisches Institut, Ruprecht-Karls-Universität Heidelberg, Heidelberg; (c) ZITI Institut für technische Informatik, Ruprecht-Karls-Universität Heidelberg, Mannheim, Germany

59 Faculty of Applied Information Science, Hiroshima Institute of Technology, Hiroshima, Japan

60 Department of Physics, Indiana University, Bloomington IN, United States of America

61 Institut für Astro- und Teilchenphysik, Leopold-Franzens-Universität, Innsbruck, Austria

62 University of Iowa, Iowa City IA, United States of America

63 Department of Physics and Astronomy, Iowa State University, Ames IA, United States of America

64 Joint Institute for Nuclear Research, JINR Dubna, Dubna, Russia

65 KEK, High Energy Accelerator Research Organization, Tsukuba, Japan

66 Graduate School of Science, Kobe University, Kobe, Japan

67 Faculty of Science, Kyoto University, Kyoto, Japan

68 Kyoto University of Education, Kyoto, Japan

69 Department of Physics, Kyushu University, Fukuoka, Japan

70 Instituto de Física La Plata, Universidad Nacional de La Plata and CONICET, La Plata, Argentina

71 Physics Department, Lancaster University, Lancaster, United Kingdom

72 (a) INFN Sezione di Lecce; (b) Dipartimento di Matematica e Fisica, Università del Salento, Lecce, Italy

73 Oliver Lodge Laboratory, University of Liverpool, Liverpool, United Kingdom

74 Department of Physics, Jožef Stefan Institute and University of Ljubljana, Ljubljana, Slovenia

75 School of Physics and Astronomy, Queen Mary University of London, London, United Kingdom

76 Department of Physics, Royal Holloway University of London, Surrey, United Kingdom

77 Department of Physics and Astronomy, University College London, London, United Kingdom

78 Louisiana Tech University, Ruston LA, United States of America

79 Laboratoire de Physique Nucléaire et de Hautes Energies, UPMC and Université Paris-Diderot and CNRS/IN2P3, Paris, France

80 Fysiska institutionen, Lunds universitet, Lund, Sweden

81 Departamento de Fisica Teorica C-15, Universidad Autonoma de Madrid, Madrid, Spain

82 Institut für Physik, Universität Mainz, Mainz, Germany

83 School of Physics and Astronomy, University of Manchester, Manchester, United Kingdom

84 CPPM, Aix-Marseille Université and CNRS/IN2P3, Marseille, France

85 Department of Physics, University of Massachusetts, Amherst MA, United States of America

86 Department of Physics, McGill University, Montreal QC, Canada

87 School of Physics, University of Melbourne, Victoria, Australia

88 Department of Physics, The University of Michigan, Ann Arbor MI, United States of America

89 Department of Physics and Astronomy, Michigan State University, East Lansing MI, United States of America

90 (a) INFN Sezione di Milano; ${ }^{(b)}$ Dipartimento di Fisica, Università di Milano, Milano, Italy

91 B.I. Stepanov Institute of Physics, National Academy of Sciences of Belarus, Minsk, Republic of Belarus

92 National Scientific and Educational Centre for Particle and High Energy Physics, Minsk, Republic of Belarus

93 Department of Physics, Massachusetts Institute of Technology, Cambridge MA, United States of America

94 Group of Particle Physics, University of Montreal, Montreal QC, Canada

95 P.N. Lebedev Institute of Physics, Academy of Sciences, Moscow, Russia

96 Institute for Theoretical and Experimental Physics (ITEP), Moscow, Russia

97 Moscow Engineering and Physics Institute (MEPhI), Moscow, Russia

98 D.V.Skobeltsyn Institute of Nuclear Physics, M.V.Lomonosov Moscow State University, Moscow, Russia

99 Fakultät für Physik, Ludwig-Maximilians-Universität München, München, Germany

100 Max-Planck-Institut für Physik(Werner-Heisenberg-Institut), München, Germany

101 Nagasaki Institute of Applied Science, Nagasaki, Japan

102 Graduate School of Science and Kobayashi-Maskawa Institute, Nagoya University, Nagoya, Japan

103 (a) INFN Sezione di Napoli; (b) Dipartimento di Fisica, Università di Napoli, Napoli, Italy

104 Department of Physics and Astronomy, University of New Mexico, Albuquerque NM, United States of America 
105 Institute for Mathematics, Astrophysics and Particle Physics, Radboud University Nijmegen/Nikhef, Nijmegen, Netherlands

106 Nikhef National Institute for Subatomic Physics and University of Amsterdam, Amsterdam, Netherlands

107 Department of Physics, Northern Illinois University, DeKalb IL, United States of America

108 Budker Institute of Nuclear Physics, SB RAS, Novosibirsk, Russia

109 Department of Physics, New York University, New York NY, United States of America

110 Ohio State University, Columbus OH, United States of America

111 Faculty of Science, Okayama University, Okayama, Japan

112 Homer L. Dodge Department of Physics and Astronomy, University of Oklahoma, Norman OK, United States of America

113 Department of Physics, Oklahoma State University, Stillwater OK, United States of America

114 Palacký University, RCPTM, Olomouc, Czech Republic

115 Center for High Energy Physics, University of Oregon, Eugene OR, United States of America

116 LAL, Université Paris-Sud and CNRS/IN2P3, Orsay, France

117 Graduate School of Science, Osaka University, Osaka, Japan

118 Department of Physics, University of Oslo, Oslo, Norway

119 Department of Physics, Oxford University, Oxford, United Kingdom

$120{ }^{(a)}$ INFN Sezione di Pavia; ${ }^{(b)}$ Dipartimento di Fisica, Università di Pavia, Pavia, Italy

121 Department of Physics, University of Pennsylvania, Philadelphia PA, United States of America

122 Petersburg Nuclear Physics Institute, Gatchina, Russia

123 (a) INFN Sezione di Pisa; ${ }^{(b)}$ Dipartimento di Fisica E. Fermi, Università di Pisa, Pisa, Italy

124 Department of Physics and Astronomy, University of Pittsburgh, Pittsburgh PA, United States of America

125 (a) Laboratorio de Instrumentacao e Fisica Experimental de Particulas - LIP, Lisboa; ${ }^{(b)}$ Faculdade de Ciências, Universidade de Lisboa, Lisboa; ${ }^{(c)}$ Department of Physics, University of Coimbra, Coimbra; ${ }^{(d)}$ Centro de Física Nuclear da Universidade de Lisboa, Lisboa; ${ }^{(e)}$ Departamento de Fisica, Universidade do Minho, Braga; ${ }^{(f)}$ Departamento de Fisica Teorica y del Cosmos and CAFPE, Universidad de Granada, Granada (Spain); ${ }^{(g)}$ Dep Fisica and CEFITEC of Faculdade de Ciencias e Tecnologia, Universidade Nova de Lisboa, Caparica, Portugal

126 Institute of Physics, Academy of Sciences of the Czech Republic, Praha, Czech Republic

127 Czech Technical University in Prague, Praha, Czech Republic

128 Faculty of Mathematics and Physics, Charles University in Prague, Praha, Czech Republic

129 State Research Center Institute for High Energy Physics, Protvino, Russia

130 Particle Physics Department, Rutherford Appleton Laboratory, Didcot, United Kingdom

131 Physics Department, University of Regina, Regina SK, Canada

132 Ritsumeikan University, Kusatsu, Shiga, Japan

133 (a) INFN Sezione di Roma; ${ }^{(b)}$ Dipartimento di Fisica, Sapienza Università di Roma, Roma, Italy

134 (a) INFN Sezione di Roma Tor Vergata; ${ }^{(b)}$ Dipartimento di Fisica, Università di Roma Tor Vergata, Roma, Italy

135 (a) INFN Sezione di Roma Tre; ${ }^{(b)}$ Dipartimento di Matematica e Fisica, Università Roma Tre, Roma, Italy

136 (a) Faculté des Sciences Ain Chock, Réseau Universitaire de Physique des Hautes Energies - Université Hassan II, Casablanca; ${ }^{(b)}$ Centre National de l'Energie des Sciences Techniques Nucleaires, Rabat; ${ }^{(c)}$ Faculté des Sciences Semlalia, Université Cadi Ayyad, LPHEA-Marrakech; ${ }^{(d)}$ Faculté des Sciences, Université Mohamed Premier and LPTPM, Oujda; ${ }^{(e)}$ Faculté des sciences, Université Mohammed V-Agdal, Rabat, Morocco

137 DSM/IRFU (Institut de Recherches sur les Lois Fondamentales de l'Univers), CEA Saclay (Commissariat à l'Energie Atomique et aux Energies Alternatives), Gif-sur-Yvette, France

138 Santa Cruz Institute for Particle Physics, University of California Santa Cruz, Santa Cruz CA, United States of America

139 Department of Physics, University of Washington, Seattle WA, United States of America

140 Department of Physics and Astronomy, University of Sheffield, Sheffield, United Kingdom

141 Department of Physics, Shinshu University, Nagano, Japan

142 Fachbereich Physik, Universität Siegen, Siegen, Germany

143 Department of Physics, Simon Fraser University, Burnaby BC, Canada

144 SLAC National Accelerator Laboratory, Stanford CA, United States of America 
145 (a) Faculty of Mathematics, Physics \& Informatics, Comenius University, Bratislava; ${ }^{(b)}$ Department of Subnuclear Physics, Institute of Experimental Physics of the Slovak Academy of Sciences, Kosice, Slovak Republic

146 (a) Department of Physics, University of Cape Town, Cape Town; ${ }^{(b)}$ Department of Physics, University of Johannesburg, Johannesburg; ${ }^{(c)}$ School of Physics, University of the Witwatersrand, Johannesburg, South Africa

147 (a) Department of Physics, Stockholm University; ${ }^{(b)}$ The Oskar Klein Centre, Stockholm, Sweden

148 Physics Department, Royal Institute of Technology, Stockholm, Sweden

149 Departments of Physics \& Astronomy and Chemistry, Stony Brook University, Stony Brook NY, United States of America

150 Department of Physics and Astronomy, University of Sussex, Brighton, United Kingdom

151 School of Physics, University of Sydney, Sydney, Australia

152 Institute of Physics, Academia Sinica, Taipei, Taiwan

153 Department of Physics, Technion: Israel Institute of Technology, Haifa, Israel

154 Raymond and Beverly Sackler School of Physics and Astronomy, Tel Aviv University, Tel Aviv, Israel

155 Department of Physics, Aristotle University of Thessaloniki, Thessaloniki, Greece

156 International Center for Elementary Particle Physics and Department of Physics, The University of Tokyo, Tokyo, Japan

157 Graduate School of Science and Technology, Tokyo Metropolitan University, Tokyo, Japan

158 Department of Physics, Tokyo Institute of Technology, Tokyo, Japan

159 Department of Physics, University of Toronto, Toronto ON, Canada

160 (a) TRIUMF, Vancouver BC; ${ }^{(b)}$ Department of Physics and Astronomy, York University, Toronto ON, Canada

161 Faculty of Pure and Applied Sciences, University of Tsukuba, Tsukuba, Japan

162 Department of Physics and Astronomy, Tufts University, Medford MA, United States of America

163 Centro de Investigaciones, Universidad Antonio Narino, Bogota, Colombia

164 Department of Physics and Astronomy, University of California Irvine, Irvine CA, United States of America

165 (a) INFN Gruppo Collegato di Udine, Sezione di Trieste, Udine; ${ }^{(b)}$ ICTP, Trieste; ${ }^{(c)}$ Dipartimento di Chimica, Fisica e Ambiente, Università di Udine, Udine, Italy

166 Department of Physics, University of Illinois, Urbana IL, United States of America

167 Department of Physics and Astronomy, University of Uppsala, Uppsala, Sweden

168 Instituto de Física Corpuscular (IFIC) and Departamento de Física Atómica, Molecular y Nuclear and Departamento de Ingeniería Electrónica and Instituto de Microelectrónica de Barcelona (IMB-CNM),

University of Valencia and CSIC, Valencia, Spain

169 Department of Physics, University of British Columbia, Vancouver BC, Canada

170 Department of Physics and Astronomy, University of Victoria, Victoria BC, Canada

171 Department of Physics, University of Warwick, Coventry, United Kingdom

172 Waseda University, Tokyo, Japan

173 Department of Particle Physics, The Weizmann Institute of Science, Rehovot, Israel

174 Department of Physics, University of Wisconsin, Madison WI, United States of America

175 Fakultät für Physik und Astronomie, Julius-Maximilians-Universität, Würzburg, Germany

176 Fachbereich C Physik, Bergische Universität Wuppertal, Wuppertal, Germany

177 Department of Physics, Yale University, New Haven CT, United States of America

178 Yerevan Physics Institute, Yerevan, Armenia

179 Centre de Calcul de l'Institut National de Physique Nucléaire et de Physique des Particules (IN2P3), Villeurbanne, France

a Also at Department of Physics, King's College London, London, United Kingdom

${ }^{b}$ Also at Institute of Physics, Azerbaijan Academy of Sciences, Baku, Azerbaijan

c Also at Particle Physics Department, Rutherford Appleton Laboratory, Didcot, United Kingdom

$d$ Also at TRIUMF, Vancouver BC, Canada

e Also at Department of Physics, California State University, Fresno CA, United States of America

$f$ Also at CPPM, Aix-Marseille Université and CNRS/IN2P3, Marseille, France 
$g$ Also at Università di Napoli Parthenope, Napoli, Italy

$h$ Also at Institute of Particle Physics (IPP), Canada

${ }^{i}$ Also at Department of Physics, St. Petersburg State Polytechnical University, St. Petersburg, Russia

$j$ Also at Department of Financial and Management Engineering, University of the Aegean, Chios, Greece

${ }^{k}$ Also at Louisiana Tech University, Ruston LA, United States of America

$l$ Also at Institucio Catalana de Recerca i Estudis Avancats, ICREA, Barcelona, Spain

$m$ Also at CERN, Geneva, Switzerland

$n$ Also at Ochadai Academic Production, Ochanomizu University, Tokyo, Japan

o Also at Manhattan College, New York NY, United States of America

$p$ Also at Novosibirsk State University, Novosibirsk, Russia

$q$ Also at Institute of Physics, Academia Sinica, Taipei, Taiwan

${ }^{r}$ Also at LAL, Université Paris-Sud and CNRS/IN2P3, Orsay, France

$s$ Also at School of Physics and Engineering, Sun Yat-sen University, Guangzhou, China

${ }^{t}$ Also at Academia Sinica Grid Computing, Institute of Physics, Academia Sinica, Taipei, Taiwan

"Also at Laboratoire de Physique Nucléaire et de Hautes Energies, UPMC and Université Paris-Diderot and CNRS/IN2P3, Paris, France

$\checkmark$ Also at School of Physical Sciences, National Institute of Science Education and Research, Bhubaneswar, India

w Also at Dipartimento di Fisica, Sapienza Università di Roma, Roma, Italy

$x$ Also at Moscow Institute of Physics and Technology State University, Dolgoprudny, Russia

y Also at section de Physique, Université de Genève, Geneva, Switzerland

$z$ Also at Department of Physics, The University of Texas at Austin, Austin TX, United States of America

a A Also at Institute for Particle and Nuclear Physics, Wigner Research Centre for Physics, Budapest, Hungary

ab Also at International School for Advanced Studies (SISSA), Trieste, Italy

ac Also at Department of Physics and Astronomy, University of South Carolina, Columbia SC, United States of America

ad Also at Faculty of Physics, M.V.Lomonosov Moscow State University, Moscow, Russia

ae Also at Physics Department, Brookhaven National Laboratory, Upton NY, United States of America

af Also at Moscow Engineering and Physics Institute (MEPhI), Moscow, Russia

ag Also at Department of Physics, Oxford University, Oxford, United Kingdom

ah Also at Department of Physics, Nanjing University, Jiangsu, China

ai Also at Institut für Experimentalphysik, Universität Hamburg, Hamburg, Germany

aj Also at Department of Physics, The University of Michigan, Ann Arbor MI, United States of America

ak Also at Discipline of Physics, University of KwaZulu-Natal, Durban, South Africa

* Deceased 